\title{
EXISTENCE AND LIPSCHITZ STABILITY FOR $\alpha$-DISSIPATIVE SOLUTIONS OF THE TWO-COMPONENT HUNTER-SAXTON SYSTEM
}

\author{
KATRIN GRUNERT AND ANDERS NORDLI
}

\begin{abstract}
We establish the concept of $\alpha$-dissipative solutions for the twocomponent Hunter-Saxton system under the assumption that either $\alpha(x)=1$ or $0 \leq \alpha(x)<1$ for all $x \in \mathbb{R}$. Furthermore, we investigate the Lipschitz stability of solutions with respect to time by introducing a suitable parametrized family of metrics in Lagrangian coordinates. This is necessary due to the fact that the solution space is not invariant with respect to time.
\end{abstract}

\section{INTRODUCTION}

In this paper we investigate the existence and Lipschitz stability of solutions of the initial value problem of the two-component Hunter-Saxton (2HS) system on the line, which is given by

$$
\begin{aligned}
u_{t}(x, t)+u u_{x}(x, t) & =\frac{1}{4}\left(\int_{-\infty}^{x}\left(u_{x}(z, t)^{2}+\rho(z, t)^{2}\right) \mathrm{d} z\right. \\
& \left.\quad-\int_{x}^{\infty}\left(u_{x}(z, t)^{2}+\rho(z, t)^{2}\right) \mathrm{d} z\right), \\
\rho_{t}(x, t)+(u \rho)_{x}(x, t) & =0 .
\end{aligned}
$$

It has been derived by Pavlov as a model of non-dissipative dark matter [17, but can also be viewed as a high frequency limit of the two-component Camassa-Holm system describing water waves [8, 18]. Moreover, it is a generalization of the well known Hunter-Saxton equation

$$
u_{t}(x, t)+u u_{x}(x, t)=\frac{1}{4}\left(\int_{-\infty}^{x} u_{x}(z, t)^{2} \mathrm{~d} z-\int_{x}^{\infty} u_{x}(z, t)^{2} \mathrm{~d} z\right),
$$

which has been introduced by Hunter and Saxton as a model of the director field of a nematic liquid crystal [14.

Solutions of the 2HS system develop singularities in finite time, even for smooth initial data [16, 18. The appearance of singularities, known as wave breaking, means that $u_{x}$ tends pointwise to $-\infty$ while $u$ remains bounded and continuous. The phenomenon is illustrated in the following example.

2010 Mathematics Subject Classification. Primary: 35Q53, 35B35; Secondary: 37L05, 37L15.

Key words and phrases. two-component Hunter-Saxton system, $\alpha$-dissipative solutions, Lipschitz stability.

Research supported by the grant Waves and Nonlinear Phenomena (WaNP) from the Research Council of Norway. 


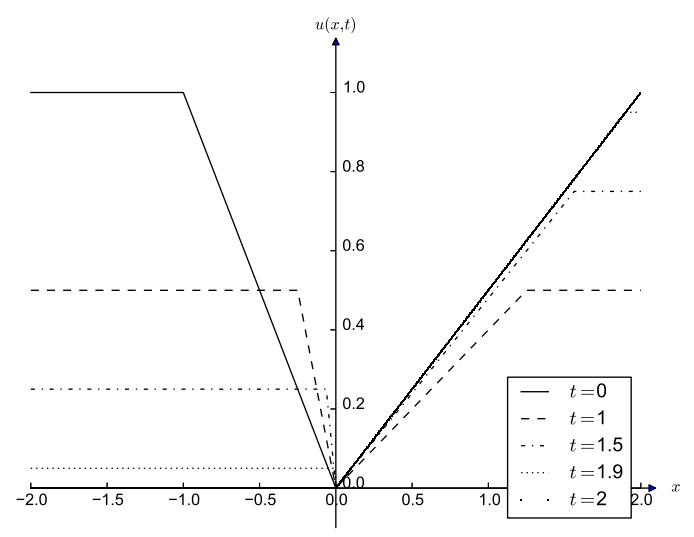

Figure 1. A plot of $u$ in Example 1.1 for $t=0,1,1.5,1.9,2$.

Example 1.1. Let $t \in[0,2)$ and let the functions $u$ and $\rho$ be defined by

$$
\begin{aligned}
& u(x, t)= \begin{cases}-\frac{1}{2} t+1, & x \leq-\left(1-\frac{1}{2} t\right)^{2}, \\
-\frac{1}{-\frac{1}{2} t+1} x, & -\left(1-\frac{1}{2} t\right)^{2} \leq x \leq 0, \\
\frac{t}{\frac{1}{2} t^{2}+2} x, & 0 \leq x \leq \frac{1}{4} t^{2}+1, \\
\frac{1}{2} t, & \frac{1}{4} t^{2}+1 \leq x,\end{cases} \\
& \rho(x, t)= \begin{cases}0, & x \leq 0, \\
\frac{1}{4} t^{2}+1 & 0<x \leq \frac{1}{4} t^{2}+1, \\
0, & \frac{1}{4} t^{2}+1<x .\end{cases}
\end{aligned}
$$

Then $(u, \rho)$ is a weak solution of (1.1) for $t \in[0,2)$. Note that $u_{x}(0, t) \rightarrow-\infty$ as $t \rightarrow 2^{-}$, which in particular means that wave breaking occurs. We can define the energy of the system at time $t$ to be given by

$$
\int_{\mathbb{R}}\left(u_{x}^{2}(x, t)+\rho^{2}(x, t)\right) \mathrm{d} x=2,
$$

which is constant in time, even up to the point $t=2$. The energy contained in the interval $-\frac{1}{4} t^{2}+t-1 \leq x \leq 0$, given by $\int_{-\frac{1}{4} t^{2}+t-1}^{0}\left(u_{x}^{2}+\rho^{2}\right) \mathrm{d} x=1$, is also conserved. Thus a finite amount of energy is being concentrated in a single point as $t \rightarrow 2^{-}$.

As seen in Example 1.1 part of the energy $\int_{\mathbb{R}}\left(u_{x}^{2}+\rho^{2}\right) \mathrm{d} x$ is concentrated at a single point at wave breaking. This illustrates that the energy density in general is not absolutely continuous, but a positive, finite Radon measure. Nevertheless, the total energy remains constant in time as $t \rightarrow 2^{-}$. Hence $u_{x}$ and $\rho$ remain in $L^{2}(\mathbb{R})$ even if $u_{x}$ tends to minus infinity and the energy can be described by the cumulative distribution function of a positive, finite Radon measure.

To continue solutions past wave breaking is a delicate issue since weak solutions are not unique afterwards. A property that the $2 \mathrm{HS}$ system shares with the HunterSaxton equation [1, 4, 5, 14, 15, 19, the Camassa-Holm equation [2, 3, 6, 13, and the two-component Camassa-Holm system [9]. To be more precise, there are 
infinitely many ways to extend a local solution to a global one past wave breaking by manipulating the concentrated energy at breaking time. In particular, there has been shown much interest in two classes of weak solutions, namely dissipative solutions and conservative solutions. On the one hand one could ignore the part of the energy that concentrates on a set of measure zero in the continuation, which yields dissipative solutions. On the other hand one could continue by letting the concentrated energy back into the system, which would give conservative solutions. In practice that would amount to defining $u$ and $\rho$ by the formulas in Example 1.1 even for $t>2$. Thus it is essential to include the energy in our sets of variables, when constructing global conservative solutions. Existence of dissipative solutions has been proven in [18, while existence and stability of conservative solutions has been shown in [16. Here we generalize and provide a unified approach to the notions of dissipative and conservative weak solutions.

We will work within the novel concept of $\alpha$-dissipative solutions, which has been introduced in [12] in the context of the two-component Camassa-Holm system for the case $\alpha$ being constant. Here we consider a more general case. Given a Lipschitz continuous function of space, $\alpha: \mathbb{R} \rightarrow[0,1]$, an $\alpha$-dissipative solution will dissipate an $\alpha$-fraction of the energy concentrated on a set of measure zero at wave breaking. Dissipative and conservative solutions are recovered as special cases with $\alpha=1$ and $\alpha=0$, respectively. Here we will construct $\alpha$-dissipative solutions and prove Lipschitz stability of the constructed solutions.

We will solve the $2 \mathrm{HS}$ system by a generalized method of characteristics. The method will be similar to the one for conservative solutions [16] and dissipative solutions [18. As long as solutions $(u, \rho)$ stay smooth we can define the corresponding Lagrangian variables $(y, U, r, V)$ by

$$
y_{t}(\xi, t)=u(y(\xi, t), t),
$$

and

$$
\begin{aligned}
U(\xi, t) & =u(y(\xi, t), t), \\
V(\xi, t) & =\int_{-\infty}^{y(\xi, t)} u_{x}(x, t)^{2}+\rho(x, t)^{2} d x=0, \\
r(\xi, t) & =\rho(y(\xi, t), t) y_{\xi}(\xi, t),
\end{aligned}
$$

where $\xi$ is a parameter that determines the initial value of the characteristics. Then

$$
\begin{aligned}
U_{t}(\xi, t) & =\frac{1}{2} V(\xi, t)-\frac{1}{4} \lim _{\xi \rightarrow \infty} V(\xi, t), \\
V_{t}(\xi, t) & =0 \\
r_{t}(\xi, t) & =0
\end{aligned}
$$

Wave breaking happens precisely where different characteristic curves $y(\xi, t), y\left(\xi^{\prime}, t\right)$ meet, and one can choose which solution to obtain by manipulating the energy density $V_{\xi}(\xi, t)$ at wave breaking. The time of wave breaking $\tau(\xi)$ can be determined initially and is given by

$$
\tau(\xi)=-2 \frac{y_{0, \xi}(\xi)}{U_{0, \xi}(\xi)}
$$

To obtain dissipative solutions one sets $V_{\xi}(\xi, t)=0$ after wave breaking, which implies that the characteristics stick together after they meet. One can solve the 
resulting system explicitly as shown by Wunsch [18. If one on the other hand keeps $V_{\xi}(\xi, t)$ constant across wave breaking one gets conservative solutions [16. For dissipative solutions one can always choose $y(\xi, 0)=\xi$, while for conservative solutions this choice is not possible since energy can then be concentrated on a set of measure zero initially. The main difficulty with $\alpha$-dissipative solutions compared to conservative or dissipative solutions is that it is not known a priori how the energy density is manipulated at wave breaking. After wave breaking we have $V_{\xi}(\xi, t)=$ $(1-\alpha(y(\xi, \tau(\xi)))) V_{0, \xi}(\xi)$. Since the time evolution of $y(\xi, t)$ depends heavily on the total amount of energy in the system, the points $y(\xi, \tau(\xi))$ where wave breaking happens will depend heavily on all other points where wave breaking has previously happened and cannot be computed explicitly. In Section 2 we reformulate the $2 \mathrm{HS}$ system in Lagrangian coordinates and prove the existence of $\alpha$-dissipative solutions in Lagrangian coordinates.

Similar to the case of conservative solutions, for $\alpha$-dissipative solutions parts of the energy can concentrate on a set of measure zero and later be given back into the system, hence one has to be careful when going from the Eulerian to the Lagrangian description of the system. Since we cannot choose $y(\xi, 0)=\xi$ we need a systematic way of going from Eulerian coordinates $(u, \rho, \mu)$ to Lagrangian variables $(y, U, r, V)$, and vice versa. We will use the mappings developed for the Camassa-Holm equation in 9, 12, 13, and modify them to the solution spaces for the 2HS system [4, 16. Even though we cannot use $y(\xi, 0)=\xi$, there is no unique way to define $y(\xi, 0)=y_{0}(\xi)$. Thus there is some redundancy in the Lagrangian variables, and we can identify a group of homeomorphisms $\xi \mapsto f(\xi)$ that acts on the Lagrangian variables and identifies equivalence classes which are in one-to-one correspondence to the Eulerian coordinates. We will develop the mappings between Eulerian and Lagrangian coordinates, and use those mappings to prove existence of $\alpha$-dissipative solutions in Eulerian coordinates in Section 3 .

In Section 4 , we turn to the Lipschitz stability of solutions. Here the redundancy will cause some problems since two solutions may differ in Lagrangian coordinates, but coincide in Eulerian coordinates. To overcome this obstacle we must create a metric in Lagrangian coordinates that gives zero distance between solutions that map to the same Eulerian solution. Such a metric was created in 4 for conservative solutions of (1.2). Moreover, for metrics in Lagrangian coordinates to induce metrics in Eulerian coordinates we need that there is a bijection from Lagrangian coordinates to Eulerian coordinates. For this to be the case we must restrict $\alpha$ to either $\alpha \equiv 1$, that is dissipative solutions, or $\alpha: \mathbb{R} \rightarrow[0,1)$.

Since the size of the discontinuity in the energy at wave breaking is unknown initially, it is more difficult to establish stability of solutions in this case than in the conservative one. To do so we will need to keep track of the amount of energy initially. Thus in addition to the energy variable $\mu$ we will need an energy variable $\nu$ such that $\mu \leq \nu$, and $\mu_{0}=\nu_{0}$. In Lagrangian coordinates this corresponds to a variable $H$ such that $V_{\xi} \leq H_{\xi}$ and $V_{0, \xi}=H_{0, \xi}$ and $H_{t}(\xi, t)=0$. Hence the solution space will not be invariant with respect to time and therefore we will introduce a parametrized family of metrics $d_{\mathcal{D}^{\alpha, M}}(t, \cdot, \cdot)$ on sets with the total energy bounded by $M$. The main result of Section 4 is that the $\alpha$-dissipative solutions constructed in Section 3 are Lipschitz continuous in time with respect to the initial data. 


\section{Existence of Solutions in Lagrangian CoORdinates}

In 16] the conservative solutions to (1.1) have been constructed by rewriting the $2 \mathrm{HS}$ system as a system of differential equations in a suitable Banach space. Here we are aiming at constructing so-called $\alpha$-dissipative solutions where one takes out an $\alpha$-fraction of the concentrated energy every time wave breaking occurs. The description of these solutions is also based on a reformulation of (1.1) in Lagrangian coordinates via a generalized method of characteristics. In contrast to 12, where the concept of $\alpha$-dissipative solutions has been introduced for $\alpha$ being a constant in $[0,1]$ in the context of the two-component Camassa-Holm system, we are considering functions

$$
\alpha(x) \in W^{1, \infty}(\mathbb{R})
$$

such that

$$
\text { either } \quad 0 \leq \alpha(x)<1 \quad \text { or } \quad \alpha(x)=1 \quad \text { for all } x \in \mathbb{R} .
$$

In this section we will first introduce the concept of $\alpha$-dissipative solutions in Lagrangian coordinates and then establish their existence in this setting.

Let $\left(u_{0}, \rho_{0}\right)$ be some smooth initial data for (1.1), such that $u_{0}(x) \in L^{\infty}(\mathbb{R})$ and $u_{0, x}(x), \rho_{0}(x) \in L^{2}(\mathbb{R})$. In addition, in this case the initial characteristic $y(\xi, 0)$ can be chosen to be equal to the identity or more general to any strictly increasing function $y_{0}(\xi)$ belonging to the set of relabeling functions $G$, which will be introduced in Definition 3.4. Applying the method of characteristics, we obtain a local in time solution, by solving the initial value problem

$$
\begin{aligned}
y_{t}(\xi, t) & =U(\xi, t), \\
U_{t}(\xi, t) & =\frac{1}{2} H(\xi, t)-\frac{1}{4} \lim _{\xi \rightarrow \infty} H(\xi, t), \\
H_{t}(\xi, t) & =0 \\
r_{t}(\xi, t) & =0
\end{aligned}
$$

with initial data

$$
\begin{aligned}
y(\xi, 0) & =y_{0}(\xi) \\
U(\xi, 0) & =u_{0}(y(\xi, 0))=u_{0}\left(y_{0}(\xi)\right) \\
H(\xi, 0) & =\int_{-\infty}^{y(\xi, 0)}\left(u_{0, x}^{2}(z)+\rho_{0}^{2}(z)\right) \mathrm{d} z=\int_{-\infty}^{y_{0}(\xi)}\left(u_{0, x}^{2}(z)+\rho_{0}^{2}(z)\right) \mathrm{d} z \\
r(\xi, 0) & =\rho_{0}(y(\xi, 0)) y_{\xi}(\xi, 0)=\rho_{0}\left(y_{0}(\xi)\right) y_{0, \xi}(\xi) .
\end{aligned}
$$

This system coincides with the one for conservative solutions and is valid until wave breaking occurs for the first time and energy concentrates on sets of measure zero. This happens when $y_{\xi}(\xi, t)=0$ for some $\xi$. To study this phenomenon in detail we differentiate the above system with respect to $\xi$ and get for each $\xi \in \mathbb{R}$ the following closed system of linear ordinary differential equations

$$
\begin{aligned}
y_{\xi, t}(\xi, t) & =U_{\xi}(\xi, t) \\
U_{\xi, t}(\xi, t) & =\frac{1}{2} H_{\xi}(\xi, t) \\
H_{\xi, t}(\xi, t) & =0,
\end{aligned}
$$


which can be solved explicitly. In particular, one has

$$
y_{\xi}(\xi, t)=y_{0, \xi}(\xi)+U_{0, \xi}(\xi) t+\frac{1}{4} H_{0, \xi}(\xi) t^{2} .
$$

Since $y_{0}(\xi)$ is strictly increasing and both $y_{0, \xi}(\xi)$ and $H_{0, \xi}(\xi)$ are positive for all $\xi \in \mathbb{R}$, wave breaking can only occur when $U_{0, \xi}(\xi)<0$. A closer look reveals that, if wave breaking occurs the breaking time $\tau(\xi)$ is given by

$$
\tau(\xi)=2 \frac{-U_{0, \xi}(\xi) \pm \sqrt{U_{0, \xi}(\xi)^{2}-y_{0, \xi}(\xi) H_{0, \xi}(\xi)}}{H_{0, \xi}(\xi)} .
$$

and since wave breaking can occur only once, we must have

$$
y_{0, \xi}(\xi) H_{0, \xi}(\xi)=U_{0, \xi}(\xi)^{2} .
$$

Comparing (2.7) with (2.3) yields, in addition, that wave breaking will occur at all points $\xi \in \mathbb{R}$ such that $U_{0, \xi}(\xi)<0$ and $r_{0}(\xi)=0$. The corresponding wave breaking time $\tau(\xi)$ is given by

$$
\tau(\xi)=-2 \frac{U_{0, \xi}(\xi)}{H_{0, \xi}(\xi)}=-2 \frac{y_{0, \xi}(\xi)}{U_{0, \xi}(\xi)} .
$$

Furthermore, it should be noted that

$$
U_{\xi}(\xi, t) \uparrow 0 \quad \text { and } \quad y_{\xi}(\xi, t) \downarrow 0 \quad \text { as } t \rightarrow \tau(\xi)-.
$$

We can now turn to the continuation of the solution past the first time wave breaking occurs. If wave breaking occurs at a point $(\xi, t)$ in Lagrangian coordinates then it occurs in Eulerian coordinates at the point $(y(\xi, t), t)$, Moreover, the energy density at any point $(\xi, t)$ in the conservative case is given by $H_{\xi}(\xi, t)$. Thus taking out an $\alpha$-fraction of the energy concentrated at a point $(\xi, \tau(\xi))$ corresponds to replacing $H_{\xi}(\xi, t)$ by

$$
V_{\xi}(\xi, t)= \begin{cases}H_{0, \xi}(\xi), & \text { if } t<\tau(\xi), \\ \left(1-\alpha(y(\xi, \tau(\xi))) H_{0, \xi}(\xi),\right. & \text { if } t \geq \tau(\xi)\end{cases}
$$

on the right hand side of (2.2a)

Before defining $\alpha$-dissipative solutions in Lagrangian coordinates rigorously, it is important to note that our introduction of the solution concept is heuristic, in that sense that we assumed that the initial data is smooth and no wave breaking occurs at time $t=0$. This limitations will be overcome in the next section, where we focus on the interplay between Eulerian and Lagrangian coordinates.

Let $E_{1}$ be the Banach space defined by

$$
E_{1}=\left\{f \in L^{\infty}(\mathbb{R}) \mid f^{\prime} \in L^{2}(\mathbb{R}) \text { and } \lim _{x \rightarrow-\infty} f(x)=0\right\},
$$

equipped with the norm $\|f\|_{E_{1}}=\|f\|_{\infty}+\left\|f^{\prime}\right\|_{2}$, and $E_{2}$ the Banach space given by

$$
E_{2}=\left\{f \in L^{\infty}(\mathbb{R}) \mid f^{\prime} \in L^{2}(\mathbb{R})\right\},
$$

equipped with the norm $\|f\|_{E_{2}}=\|f\|_{\infty}+\left\|f^{\prime}\right\|_{2}$.

In addition, let $B$ be the Banach space $B=E_{2} \times E_{2} \times E_{1} \times L^{2}(\mathbb{R}) \times E_{1}$ with the norm

$$
\left\|\left(f_{1}, f_{2}, f_{3}, f_{4}, f_{5}\right)\right\|_{B}=\left\|f_{1}\right\|_{E_{2}}+\left\|f_{2}\right\|_{E_{2}}+\left\|f_{3}\right\|_{E_{1}}+\left\|f_{4}\right\|_{2}+\left\|f_{5}\right\|_{E_{1}} .
$$

Then the set of Lagrangian coordinates $\mathcal{F}^{\alpha}$ is given as follows. 
Definition 2.1 (Lagrangian coordinates). The set $\mathcal{F}^{\alpha}$ consists of all $X=(y, U, H, r, V)$, such that $(y-\mathrm{id}, U, H, r, V) \in B$, and

(i) $y-\mathrm{id}, U, H, V \in W^{1, \infty}(\mathbb{R}), r \in L^{\infty}(\mathbb{R})$,

(ii) $0 \leq y_{\xi}, 0 \leq H_{\xi}, 0<c<H_{\xi}+y_{\xi} \quad$ almost everwhere,

(iii) $y_{\xi} V_{\xi}=U_{\xi}^{2}+r^{2} \quad$ almost everywhere,

(iv) $0 \leq V_{\xi} \leq H_{\xi} \quad$ almost everywhere,

(v) If $0 \leq \alpha(x)<1$ for all $x \in \mathbb{R}$,

then there exists $\kappa: \mathbb{R} \rightarrow(0,1]$ such that $V_{\xi}(\xi)=\kappa(y(\xi)) H_{\xi}(\xi)$ almost

everywhere, and $\kappa(y(\xi))=1$ whenever $U_{\xi}(\xi)<0$ or $r(\xi) \neq 0$,

(vi) If $\alpha(x)=1$ for all $x \in \mathbb{R}$, then $y_{\xi}(\xi)=0$ implies $V_{\xi}(\xi)=0$,

and $y_{\xi}(\xi)>0$ implies that $V_{\xi}(\xi)=H_{\xi}(\xi)$ almost everywhere.

The $\alpha$-dissipative solution $X(t) \in \mathcal{F}^{\alpha}$ with initial data $X(0)=X_{0} \in \mathcal{F}^{\alpha}$ to (1.1) in Lagrangian coordinates is then given by

$$
\begin{aligned}
y_{t}(\xi, t) & =U(\xi, t), \\
U_{t}(\xi, t) & =\frac{1}{2} V(\xi, t)-\frac{1}{4} \lim _{\xi \rightarrow \infty} V(\xi, t), \\
H_{t}(\xi, t) & =0 \\
r_{t}(\xi, t) & =0
\end{aligned}
$$

where

$$
V(\xi, t)=\int_{-\infty}^{\xi} H_{0, \xi}(\eta)\left(1-\mathbf{1}_{\{t \geq \tau(\eta)\}} \alpha(y(\eta, \tau(\eta)))\right) d \eta .
$$

Here the blow-up time $\tau(\xi)$ is given by

$$
\tau(\xi)= \begin{cases}0, & \text { if } y_{0, \xi}(\xi)=0, \\ -\frac{2 U_{0, \xi}(\xi)}{H_{0, \xi}(\xi)}, & \text { if } r_{0}(\xi)=0 \text { and } U_{0, \xi}(\xi)<0, \\ \infty, & \text { otherwise. }\end{cases}
$$

Note that $\tau(\xi)=0$ for some $\xi \in \mathbb{R}$, implies that $y_{0, \xi}(\xi)=0$ for some $\xi \in \mathbb{R}$ and hence wave breaking occurs at time $t=0$.

In (2.15) it is implicitly assumed that $V_{0, \xi}(\xi)=H_{0, \xi}(\xi)$ for a.e. $\xi \in \mathbb{R}$. This is a stricter condition than $(i v)$ in Definition 2.1. Thus we define the set of admissible initial data, $\mathcal{F}_{i}^{\alpha}$ as follows.

Definition 2.2. Let $\mathcal{F}_{i}^{\alpha}$ be the set of all $X \in \mathcal{F}^{\alpha}$ such that

$$
V(\xi)=H(\xi) \quad \text { for all } \xi \in \mathbb{R} \text {. }
$$

Here, it should be pointed out that all of Section 2 and 3 remain true for initial data in $\mathcal{F}^{\alpha}$. The reason for this restriction will be clarified in Section 4 .

We now turn our attention to the existence and uniqueness of solutions to the system (2.14) with initial data in $\mathcal{F}_{i}^{\alpha}$.

Lemma 2.3. To any initial data $X_{0} \in \mathcal{F}_{i}^{\alpha}$ there exists a unique solution $X \in$ $C\left(\mathbb{R}_{+}, \mathcal{F}^{\alpha}\right)$ of (2.14) with $X(0)=X_{0}$. 
Proof. Let $X_{0} \in \mathcal{F}_{i}^{\alpha}$. First we prove existence and uniqueness of solutions to (2.14) in $B$. We are going to construct a sequence of approximate solutions $X_{n}(t)$ that converge to the $\alpha$-dissipative solution $X(t)$ which satisfies $X(0)=X_{0}$. To that end define $X_{1}(t)=X_{0}$ for all $t \geq 0$. For $n \geq 2$, we take advantage of the fact that for each $\xi \in \mathbb{R}$ the breaking time $\tau(\xi)$ can be computed from the initial data, see (2.16). Indeed we define, for $n+1 \geq 2$,

$$
\beta_{n+1}(\xi, t)= \begin{cases}0, & \tau(\xi)=\infty, \\ \alpha\left(y_{n}(\xi, t)\right), & t<\tau(\xi)<\infty, \\ \alpha\left(y_{n}(\xi, \tau(\xi))\right), & \tau(\xi) \leq t,\end{cases}
$$

and $X_{n+1}(t)$ as the solution to

$$
\begin{aligned}
y_{n+1, t}(\xi, t) & =U_{n+1}(\xi, t), \\
U_{n+1, t}(\xi, t) & =\frac{1}{2} V_{n+1}(\xi, t)-\frac{1}{4} V_{n+1, \infty}(t), \\
H_{n+1, t}(\xi, t) & =0 \\
r_{n+1, t}(\xi, t) & =0 \\
V_{n+1}(\xi, t) & =\int_{-\infty}^{\xi} H_{0, \xi}(\eta)\left(1-\mathbf{1}_{\{t \geq \tau(\eta)\}} \beta_{n+1}(\eta, t)\right) d \eta,
\end{aligned}
$$

where $V_{n+1, \infty}(t)=\lim _{\xi \rightarrow \infty} V_{n+1}(\xi, t)$, with initial data $X_{n+1}(0)=X_{0}$. That $X_{n}(t) \in B$ for all $t \geq 0$ and $n \geq 1$ follows inductively by integrating the system.

To establish the existence of a limiting function $X(t)$ we use a fix point argument. First, estimate the difference between $y_{n}(t)$ and $y_{n+1}(t)$ for $0 \leq t \leq T$. From (2.18) we get that

$$
\left|\beta_{n+1}(\xi, t)-\beta_{n}(\xi, t)\right| \leq\left\|\alpha^{\prime}\right\|_{\infty} \sup _{t \in[0, T]}\left\|y_{n}(t)-y_{n-1}(t)\right\|_{\infty},
$$

which implies together with (2.19) that

$$
\begin{aligned}
\left|V_{n+1}(\xi, t)-V_{n}(\xi, t)\right| & \leq \int_{-\infty}^{\infty} H_{0, \xi}(\eta)\left|\beta_{n+1}(\eta, t)-\beta_{n}(\eta, t)\right| \mathbf{1}_{\{t \geq \tau(\eta)\}} d \eta \\
& \leq\left\|H_{0}\right\|_{\infty}\left\|\alpha^{\prime}\right\|_{\infty} \sup _{t \in[0, T]}\left\|y_{n}(t)-y_{n-1}(t)\right\|_{\infty}, \\
\left|U_{n+1}(\xi, t)-U_{n}(\xi, t)\right| & \leq \frac{1}{4}\left\|H_{0}\right\|_{\infty}\left\|\alpha^{\prime}\right\|_{\infty} \sup _{t \in[0, T]}\left\|y_{n}(t)-y_{n-1}(t)\right\|_{\infty} t, \\
\left|y_{n+1}(\xi, t)-y_{n}(\xi, t)\right| & \leq \frac{1}{8}\left\|H_{0}\right\|_{\infty}\left\|\alpha^{\prime}\right\|_{\infty} \sup _{t \in[0, T]}\left\|y_{n}(t)-y_{n-1}(t)\right\|_{\infty} t^{2} .
\end{aligned}
$$

Thus,

$$
\sup _{t \in[0, T]}\left\|y_{n+1}(t)-y_{n}(t)\right\|_{\infty} \leq \frac{1}{8}\left\|H_{0}\right\|_{\infty}\left\|\alpha^{\prime}\right\|_{\infty} \sup _{t \in[0, T]}\left\|y_{n}(t)-y_{n-1}(t)\right\|_{\infty} T^{2},
$$

and if $T$ is chosen such that $T<\sqrt{\frac{8}{\left\|\alpha^{\prime}\right\|_{\infty}\left\|_{0}\right\|_{\infty}}}$, we have a contraction on the Banach space $C\left([0, T], L^{\infty}(\mathbb{R})\right)$. In particular, there exists a unique function $y \in$ $C\left([0, T], L^{\infty}(\mathbb{R})\right)$ such that $y_{n}(\cdot, t) \rightarrow y(\cdot, t)$ in $L^{\infty}(\mathbb{R})$ for all $t \in[0, T]$. Moreover, (2.20), (2.21a), and 2.21b) imply that there exist unique $\beta, V$, and $U$ in $C\left([0, T], L^{\infty}(\mathbb{R})\right)$, such that $\beta_{n}(\cdot, t) \rightarrow \beta(\cdot, t), V_{n}(\cdot, t) \rightarrow V(\cdot, t)$, and $U_{n}(\cdot, t) \rightarrow$ 
$U(\cdot, t)$ in $L^{\infty}(\mathbb{R})$ for all $t \in[0, T]$ as well. As far as $r_{n}(\xi, t)$ and $H_{n}(\xi, t)$ are concerned, observe that $r_{n}(\xi, t)=r_{0}(\xi)$ and $H_{n}(\xi, t)=H_{0}(\xi)$ for all $n \geq 1$ and hence $r_{n}(t, \cdot) \rightarrow r_{0}(\cdot)$ in $L^{2}(\mathbb{R}) \cap L^{\infty}(\mathbb{R})$ and $H_{n}(\cdot, t) \rightarrow H_{0}(\cdot)$ in $L^{\infty}(\mathbb{R})$ for all $t \in \mathbb{R}$.

The system for the derivatives is given by

$$
\begin{aligned}
y_{n+1, \xi, t}(\xi, t) & =U_{n+1, \xi}(\xi, t), \\
U_{n+1, \xi, t}(\xi, t) & =\frac{1}{2}\left(1-\beta_{n+1}(\xi, t) \mathbf{1}_{\{t \geq \tau(\xi)\}}\right) H_{0, \xi}(\xi),
\end{aligned}
$$

and hence

$$
\begin{aligned}
\left|U_{n+1, \xi}(\xi, t)-U_{n, \xi}(\xi, t)\right| & \leq \frac{1}{2} t\left|\beta_{n+1}(\xi, t)-\beta_{n}(\xi, t)\right| H_{0, \xi}(\xi) \\
& \leq \frac{1}{2} t H_{0, \xi}\left\|\alpha^{\prime}\right\|_{\infty} \sup _{t \in[0, T]}\left\|y_{n}(t)-y_{n-1}(t)\right\|_{\infty}, \\
\left|y_{n+1, \xi}(\xi, t)-y_{n, \xi}(\xi, t)\right| & \leq \frac{1}{4} H_{0, \xi} t^{2}\left\|\alpha^{\prime}\right\|_{\infty} \sup _{t \in[0, T]}\left\|y_{n}(t)-y_{n-1}(t)\right\|_{\infty} .
\end{aligned}
$$

Since $H_{0, \xi}(\cdot) \in L^{2}(\mathbb{R}) \cap L^{\infty}(\mathbb{R})$ it follows that $U_{n, \xi}(\cdot, t) \rightarrow U_{\xi}(\cdot, t)$ and $\left(y_{n, \xi}-\right.$ $1)(\cdot, t) \rightarrow\left(y_{\xi}-1\right)(\cdot, t)$ in $L^{2}(\mathbb{R}) \cap L^{\infty}(\mathbb{R})$ for all $t \in[0, T]$. Thus we showed that to each $X_{0} \in \mathcal{F}_{i}^{\alpha}$ there exists a local solution $X(t)$ to (2.14) in $B$. In addition, we obtained that both $\left(y_{\xi}-1\right)(\cdot, t), U_{\xi}(\cdot, t)$, and $r(\cdot, t)$ belong to $L^{\infty}(\mathbb{R})$ for all $t \in[0, T]$.

The uniqueness of the solution $X(t)$ to (2.14) with $X(0)=X_{0} \in \mathcal{F}_{i}^{\alpha}$ on the interval $[0, T]$ follows by a standard contraction argument, based on estimates similar to the ones established so far.

To extend $X(t)$ from a local to a global solution observe that the upper bound on $T$ only depends on $\left\|H_{0}\right\|_{\infty}$ and $\left\|\alpha^{\prime}\right\|_{\infty}$, which are both independent of time. Thus one can repeat the above construction, with slight modifications, with initial data $X\left(\frac{1}{2} T\right)$ to obtain the unique solution on $\left[0, \frac{3}{2} T\right]$. Continuing inductively finally yields the unique, global solution $X(t) \in B$ to (2.14).

The method is illustrated in Example A.3 where the iteration (2.19) is applied to some multipeakon initial data.

It is left to show that $X(t)$ satisfies properties $(i i)-(v i)$ in Definition 2.1. By definition, see (2.15), we have that

$$
V_{\xi}(\xi, t)= \begin{cases}H_{0, \xi}(\xi), & \text { if } t<\tau(\xi), \\ (1-\alpha(y(\xi, \tau(\xi)))) H_{0, \xi}(\xi), & \text { if } t \geq \tau(\xi),\end{cases}
$$

and hence properties $(i v)-(v i)$ in Definition 2.1 hold since wave breaking happens when $U_{\xi}(\xi, t)=0$ and $r(\xi, t)=0$. It should be pointed out that in the case $\alpha=1$ we have that if $y_{\xi}(\xi, t)=0$, then $y_{\xi}\left(\xi, t^{\prime}\right)=0$ for all $t^{\prime}>t$.

Direct calculations yield for a.e. $\xi \in \mathbb{R}$, that

$$
\begin{aligned}
\frac{d}{d t} \frac{1}{y_{\xi}(\xi, t)+H_{0, \xi}(\xi)} & =-\frac{U_{\xi}(\xi, t)}{\left(y_{\xi}(\xi, t)+H_{0, \xi}(\xi)\right)^{2}} \\
& \leq \frac{\sqrt{y_{\xi} V_{\xi}(\xi, t)}}{\left(y_{\xi}(\xi, t)+H_{0, \xi}(\xi)\right)^{2}} \leq \frac{1}{2} \frac{1}{y_{\xi}(\xi, t)+H_{0, \xi}(\xi)},
\end{aligned}
$$

Applying Gronwall's inequality, we obtain

$$
\left(y_{\xi}(\xi, 0)+H_{0, \xi}(\xi)\right) e^{-\frac{1}{2} t} \leq\left(y_{\xi}(\xi, t)+H_{0, \xi}(\xi)\right)
$$

for a.e. $\xi \in \mathbb{R}$, i.e. property $(i i)$ in Definition 2.1 . 
Thus it is left to show that $y_{\xi}(\xi, t) V_{\xi}(\xi, t)=U_{\xi}^{2}(\xi, t)+r^{2}(\xi, t)$ for a.e. $\xi \in \mathbb{R}$ and $t>0$. For a.e. $\xi \in \mathbb{R}$ we have for $t<\tau(\xi)$,

$$
\begin{aligned}
U_{\xi}(\xi, t)^{2} & =\frac{1}{4} H_{0, \xi}^{2}(\xi) t^{2}+U_{0, \xi}(\xi) H_{0, \xi}(\xi) t+U_{0, \xi}(\xi)^{2}, \\
r(\xi, t)^{2} & =r_{0}^{2}(\xi), \\
y_{\xi} V_{\xi}(\xi, t) & =\frac{1}{4} H_{0, \xi}^{2}(\xi) t^{2}+U_{0, \xi} H_{0, \xi}(\xi) t+U_{0, \xi}^{2}(\xi)+r_{0}^{2}(\xi),
\end{aligned}
$$

where we used that $V_{0, \xi} y_{0, \xi}(\xi)=U_{0, \xi}^{2}(\xi)+r_{0}^{2}(\xi)$ for a.e. $\xi \in \mathbb{R}$. For $t \geq \tau(\xi)$, we have

$$
\begin{aligned}
r_{t}(t, \xi) & =0, \\
U_{\xi}(\xi, t) & =\frac{1}{2} V_{\xi}(\xi, \tau(\xi))(t-\tau(\xi)), \\
y_{\xi}(\xi, t) & =\frac{1}{4} V_{\xi}(\xi, \tau(\xi))(t-\tau(\xi))^{2},
\end{aligned}
$$

where we used that $U_{\xi}(\xi, \tau(\xi))=0=y_{\xi}(\xi, \tau(\xi))$. Thus, $y_{\xi} V_{\xi}(\xi, t)=U_{\xi}^{2}(\xi, t)+$ $r^{2}(\xi, t)$ for a.e $\xi \in \mathbb{R}$ also in this case and Definition 2.1 (iii) holds.

A natural question, that is going to play a major role when establishing the Lipschitz stability, is if one can estimate the size of the set where wave breaking has already taken place in Lagrangian coordinates.

Corollary 2.4. Let $X_{0} \in \mathcal{F}_{i}^{\alpha}$, and denote by $X(t)$ the solution to (2.14) with initial data $X_{0}$. Then the set of all points where wave breaking has taken place within the time interval $[0, t]$ satisfies

$$
\mathrm{m}(\{\xi \mid \tau(\xi) \leq t\}) \leq \frac{\left(1+\frac{1}{4} t^{2}\right)}{c}\left\|H_{0}\right\|_{\infty}
$$

where $c$ is given by $0<c \leq y_{0, \xi}(\xi)+H_{0, \xi}(\xi)$ for a.e. $\xi \in \mathbb{R}$ (cf. Definition [2.1).

Proof. Given $\xi \in \mathbb{R}$ such that $\tau(\xi) \leq t$, then (2.14) implies that

$$
0=y_{\xi}(\xi, \tau(\xi))=y_{0, \xi}(\xi)+U_{0, \xi}(\xi) \tau(\xi)+\frac{1}{4} H_{0, \xi}(\xi) \tau(\xi)^{2}
$$

and

$$
0=U_{\xi}(\xi, \tau(\xi))=U_{0, \xi}(\xi)+\frac{1}{2} H_{0, \xi}(\xi) \tau(\xi) .
$$

Combining these two equations finally yields $0=y_{0, \xi}(\xi)-\frac{1}{4} H_{0, \xi}(\xi) \tau(\xi)^{2}$ and hence

$$
1=\frac{H_{0, \xi}(\xi)\left(1+\frac{1}{4} \tau(\xi)^{2}\right)}{y_{0, \xi}(\xi)+H_{0, \xi}(\xi)} \leq \frac{H_{0, \xi}(\xi)\left(1+\frac{1}{4} \tau(\xi)^{2}\right)}{c},
$$

where we used that there exists $c>0$ such that $y_{0, \xi}(\xi)+H_{0, \xi}(\xi) \geq c>0$ for a.e. $\xi \in \mathbb{R}$. Thus one obtains

$$
\begin{aligned}
\mathrm{m}(\{\xi \mid \tau(\xi)=t\}) & \leq \mathrm{m}(\{\xi \mid \tau(\xi) \leq t\})=\int_{\mathbb{R}} \mathbf{1}_{\{\tau(\xi) \leq t\}} d \xi \\
& \leq \frac{1+\frac{1}{4} t^{2}}{c} \int_{\mathbb{R}} H_{0, \xi}(\xi) d \xi=\frac{1+\frac{1}{4} t^{2}}{c}\left\|H_{0}\right\|_{\infty} .
\end{aligned}
$$


Note, that if $y_{0 \xi}(\xi)+H_{0, \xi}(\xi)=1$ for all $\xi \in \mathbb{R}$, the above estimate reads

$$
\mathrm{m}(\{\xi \mid \tau(\xi) \leq t\}) \leq\left(1+\frac{1}{4} t^{2}\right)\left\|H_{0}\right\|_{\infty} .
$$

\section{Existence of solutions in Eulerian COORDinates}

In Section $2 \alpha$-dissipative solutions of the reformulation of the $2 \mathrm{HS}$ system in Lagrangian coordinates, (2.14), have been constructed. The goal of this section is to establish the connection between Eulerian and Lagrangian coordinates, which will enable us to define $\alpha$-dissipative solutions in Eulerian coordinates. The strategy is to map the initial data in Eulerian coordinates to the space $\mathcal{F}^{\alpha}$, then solve (2.14), and map the solution back to Eulerian coordinates. In order to do so the space $\mathcal{D}^{\alpha}$, of solutions in Eulerian coordinates, will be defined, and mappings between $\mathcal{F}^{\alpha}$ and $\mathcal{D}^{\alpha}$ will be established. A definition of $\alpha$-dissipative solutions of (1.1) will be given and the proposed solutions will be shown to satisfy this definition.

The solution space in Eulerian coordinates is given in the next definition.

Definition 3.1 (Eulerian coordinates). Let $\mathcal{D}^{\alpha}$ be the set of all $(u, \rho, \nu, \mu)$ such that

$$
\begin{aligned}
& \text { (i) } \quad u \in L^{\infty}(\mathbb{R}), u_{x} \in L^{2}(\mathbb{R}), \\
& \text { (ii) } \rho \in L^{2}(\mathbb{R}), \\
& \text { (iii) } \quad \mu \leq \nu \in \mathcal{M}^{+}(\mathbb{R}), \\
& \text { (iv) } \quad \mu_{a c}=\left(u_{x}^{2}+\rho^{2}\right) d x, \\
& \text { (v) } \quad \text { if } 0 \leq \alpha(x)<1 \text { for all } x \in \mathbb{R}, \text { then } \frac{d \mu}{d \nu}>0, \\
& \quad \text { and } \frac{d \mu}{d \nu}(x)=1 \text { whenever } u_{x}(x)<0 \text { or } \rho(x) \neq 0, \\
& \text { (vi) } \text { if } \alpha(x)=1 \text { for all } x \in \mathbb{R}, \text { then } \mu=\nu_{a c},
\end{aligned}
$$

where $\mathcal{M}^{+}(\mathbb{R})$ denotes the set of positive, finite Radon measures on $\mathbb{R}$.

Next, the mappings between the elements in $\mathcal{D}^{\alpha}$ and the elements of $\mathcal{F}^{\alpha}$ are introduced. When the mapping $L: \mathcal{D}^{\alpha} \rightarrow \mathcal{F}^{\alpha}$ is to be defined, the critical part is how to define the characteristics $y$ in nice way, since all the other variables will follow naturally.

Definition 3.2. Let the mapping $L: \mathcal{D}^{\alpha} \rightarrow \mathcal{F}^{\alpha}$ be defined by $L((u, \rho, \nu, \mu))=$ $(y, U, H, r, V)$ where

$$
\begin{aligned}
y(\xi) & =\sup \{x \mid \nu((-\infty, x))+x<\xi\}, \\
H(\xi) & =\xi-y(\xi), \\
U(\xi) & =u \circ y(\xi), \\
r(\xi) & =(\rho \circ y(\xi)) y_{\xi}(\xi), \\
V(\xi) & =\int_{-\infty}^{\xi} \frac{d \mu}{d \nu} \circ y(\eta) H_{\xi}(\eta) d \eta .
\end{aligned}
$$

When going from $\mathcal{F}^{\alpha}$ to $\mathcal{D}^{\alpha}$ one has to be careful, since the presence of wave breaking implies that there can be $\xi$ such that $y_{\xi}(\xi)=0$. However, $y_{\xi}(\xi)=0$ 
implies that $U_{\xi}(\xi)=0$ and $r(\xi)=0$ by property (iii) in Definition 2.1 and hence one can define the mapping $M: \mathcal{F}^{\alpha} \rightarrow \mathcal{D}^{\alpha}$ as follows.

Definition 3.3. Let the mapping $M: \mathcal{F}^{\alpha} \rightarrow \mathcal{D}^{\alpha}$ be defined by $M((y, U, H, r, V))=$ $(u, \rho, \nu, \mu)$ where

$$
\begin{aligned}
u(x) & =U(\xi), \text { for any } \xi \text { such that } x=y(\xi), \\
\rho d x & =y_{\#}(r d \xi), \\
\nu & =y_{\#}\left(H_{\xi} d \xi\right), \\
\mu & =y_{\#}\left(V_{\xi} d \xi\right) .
\end{aligned}
$$

That $L$ and $M$ are well-defined follows from combining the proofs of [16, Proposition 2.6 and 2.9], [12, Definitions 3.9 and 3.10, Theorem 3.11], and [13, Theorem 3.8 and 3.11], where slightly different mappings have been considered. We therefore leave the details to the interested reader.

There are five variables in Lagrangian coordinates, while in Eulerian coordinates there are only four. Hence there cannot be a one-to-one correspondence. Indeed, there is an equivalence relation $\sim$ on $\mathcal{F}^{\alpha}$ such that $X \sim \bar{X}$ implies that $M(X)=$ $M(\bar{X})$. This equivalence relation is induced by a group action $\bullet$ by a group $G$ given in the next definition.

Definition 3.4. Let the group $G$ and the group action $\bullet$ of $G$ on $\mathcal{F}^{\alpha}$ be defined as follows.

(i) Define $G$ as the group of homeomorphisms $f: \mathbb{R} \rightarrow \mathbb{R}$ such that

$$
f-\mathrm{id} \in W^{1, \infty}(\mathbb{R}), f^{-1}-\mathrm{id} \in W^{1, \infty}(\mathbb{R}) \text {, and } f_{\xi}-1 \in L^{2}(\mathbb{R}) .
$$

(ii) Define a group action $\bullet: \mathcal{F}^{\alpha} \times G \rightarrow \mathcal{F}^{\alpha}$ by

$$
(X, f) \mapsto\left(y \circ f, U \circ f, H \circ f,(r \circ f) \cdot f_{\xi}, V \circ f\right)=X \bullet f .
$$

We will call the action of $f \in G$ on $X \in \mathcal{F}^{\alpha}$ the relabeling of $X$ by $f$.

That the group action $\bullet$ is well-defined follows the same lines as [13, Proposition 3.4 and is left to the interested reader.

As indicated above $M$ respects equivalence classes identified via relabeling.

Proposition 3.5. We have for any $X \in \mathcal{F}^{\alpha}$ and $f \in G$ that

$$
M(X \bullet f)=M(X) \text {. }
$$

Proof. The proof is a combination of the ones of [13, Theorem 3.11], 16, Proposition 2.12], and [12, Definition 3.19].

Let us look if the time evolution respects relabeling. To that end define $S_{t}$ the solution operators that maps any initial data $X_{0} \in \mathcal{F}_{i}^{\alpha}$ to its unique $\alpha$-dissipative solution $X(t)$ at time $t$.

Definition 3.6. For any $t \geq 0$ and $X_{0} \in \mathcal{F}_{i}^{\alpha}$ define $S_{t}\left(X_{0}\right)$ to be the unique $\alpha$-dissipative solution $X(t)$ at time $t$ that satisfies $X(0)=X_{0}$.

For our solution concept to be well-defined we have to prove that $S_{t}$ commutes with relabeling.

Proposition 3.7. For any $X_{0} \in \mathcal{F}_{i}^{\alpha}, f \in G$ it holds that $S_{t}\left(X_{0} \bullet f\right)=S_{t}\left(X_{0}\right) \bullet f$. 
Proof. Let $f \in G$ and $X_{0} \in \mathcal{F}_{i}^{\alpha}$ be given and denote by $X(t)$ the corresponding $\alpha$ dissipative solution, i.e. $X(t)=S_{t}\left(X_{0}\right)$. According to Definition 3.4 $\hat{X}_{0}=X_{0} \bullet f \in$ $\mathcal{F}_{i}^{\alpha}$ and the corresponding solution is given by $\hat{X}(t)=S_{t}\left(\hat{X}_{0}\right)=S_{t}\left(X_{0} \bullet f\right)$. Thus we have to show that $\hat{X}(t)=X(t) \bullet f$ for all $t \geq 0$. At initial time $t=0$ we have $\hat{X}(0)=X_{0} \bullet f=X(0) \bullet f$. For $t>0$ recall that both $X(t)$ and $\hat{X}(t)$ can be seen as the limit of sequences $X_{n}(t)$ and $\hat{X}_{n}(t)$, respectively, as defined in the proof of Lemma 2.3. In particular one has that

$$
\hat{X}(t)=\lim _{n \rightarrow \infty} \hat{X}_{n}(t) \quad \text { and } \quad X(t) \bullet f=\lim _{n \rightarrow \infty}\left(X_{n}(t) \bullet f\right) .
$$

Thus it suffices to show by induction that $\hat{X}_{n}(t)=X_{n}(t) \bullet f$ for all $n \in \mathbb{N}$ and all $t \geq 0$. First of all note that

$$
\hat{\tau}(\xi)=\tau(f(\xi)) \quad \text { for all } \xi
$$

and by definition $\hat{X}_{1}(t)=\hat{X}(0)=X_{0} \bullet f=X_{1}(t) \bullet f$ for all $t \geq 0$. For $n=2$ direct calculations yield that $\hat{X}_{2}(t)=X_{2}(t) \bullet f$. To conclude the argument, assume that

$$
\hat{X}_{n}(t)=X_{n}(t) \bullet f \quad \text { for all } t \geq 0 .
$$

Combining (2.18), (3.5) and (3.6) yields

$$
\hat{\beta}_{n+1}(\xi, t)=\beta_{n+1}(f(\xi), t) \quad \text { for all } t \geq 0,
$$

and subsequently

$$
\begin{aligned}
\hat{V}_{n+1}(\xi, t) & =\int_{-\infty}^{\xi}\left(1-\hat{\beta}_{n+1}(\eta, t) \mathbf{1}_{\{t \geq \hat{\tau}(\eta)\}}\right) \hat{H}_{0, \xi}(\eta) d \eta \\
& =\int_{-\infty}^{\xi}\left(1-\beta_{n+1}(f(\eta), t) \mathbf{1}_{\{t \geq \tau(f(\eta))\}}\right) H_{0, \xi}(f(\eta)) f_{\xi}(\eta) d \eta \\
& =\int_{-\infty}^{f(\xi)}\left(1-\beta_{n+1}(\eta, t) \mathbf{1}_{\{t \geq \tau(\eta)\}}\right) H_{0, \xi}(\eta) d \eta=V_{n+1}(f(\xi), t) .
\end{aligned}
$$

Thus (2.19) implies that $\hat{X}_{n}(t)=X_{n}(t) \bullet f$ for all $n \in \mathbb{N}$ and all $t \geq 0$.

To show that there is a one-to-one correspondence between $\mathcal{D}^{\alpha}$ and equivalence classes in $\mathcal{F}^{\alpha}$, we need to identify a subset of $\mathcal{F}^{\alpha}$ containing one representative of each equivalence class. To that end define $\mathcal{F}_{0}^{\alpha}$ by

$$
\mathcal{F}_{0}^{\alpha}=\left\{X \in \mathcal{F}^{\alpha} \mid y+H=\mathrm{id}\right\} .
$$

The following proposition states that the mappings $M$ and $L$ are inverses of each other when $M$ is restricted to $\mathcal{F}_{0}^{\alpha}$.

Proposition 3.8. The mappings $M$ and $L$ satisfy

$$
\begin{aligned}
& M \circ L=\operatorname{id}_{\mathcal{D}^{\alpha}}, \\
& L \circ M=\operatorname{id}_{\mathcal{F}_{0}^{\alpha}} .
\end{aligned}
$$

Proof. See [12, Theorem 3.11] and [13, Theorem 3.12].

Observe that $L$ maps $\mathcal{D}^{\alpha}$ to $\mathcal{F}_{0}^{\alpha}$. In addition, any initial data $\left(u_{0}, \rho_{0}, \mu_{0}, \mu_{0}\right)$ is mapped to an element in $\mathcal{F}_{i, 0}^{\alpha}=\mathcal{F}_{0}^{\alpha} \cap \mathcal{F}_{i}^{\alpha}$, which is advantageous because, among other things, (2.32) applies in this setting.

If $\bullet$ is to induce the desired equivalence relation, then to each $X \in \mathcal{F}^{\alpha}$ there must exist $f \in G$ such that $X \bullet f \in \mathcal{F}_{0}^{\alpha}$. 
Definition 3.9. Define the map $\Pi: \mathcal{F}^{\alpha} \rightarrow \mathcal{F}_{0}^{\alpha}$ by

$$
\Pi X=X \bullet(y+H)^{-1}, \quad X \in \mathcal{F}^{\alpha} .
$$

To ease the notation we write $\Pi X$, despite the fact that $\Pi$ is not a linear operator.

For the map $\Pi$ to be well-defined we need that $(y+H)^{-1} \in G$.

Proposition 3.10. Let $X \in \mathcal{F}^{\alpha}$, then $y+H \in G$ and $(y+H)^{-1} \in G$. Moreover if $X_{0} \in \mathcal{F}_{i, 0}^{\alpha}$, and $X(t)=S_{t}\left(X_{0}\right)$, then for all $t \geq 0$ we have that $e^{-\frac{1}{2} t} \leq y_{\xi}(\xi, t)+$ $H_{\xi}(\xi, t) \leq \frac{1}{4} t^{2}+t+1$ for almost every $\xi \in \mathbb{R}$.

Proof. Let $y+H=f$, we show that both $f$ and $f^{-1}$ belong to $G$. From the definition of $\mathcal{F}^{\alpha}$ we have that $f-\mathrm{id} \in W^{1, \infty}(\mathbb{R})$ with $f_{\xi}-1 \in L^{2}(\mathbb{R})$, with $c \leq f_{\xi} \leq C$ for some positive numbers $c$ and $C$. Thus there exists a Lipschitz continuous inverse $f^{-1}$ such that $f^{-1}-\mathrm{id} \in W^{1, \infty}(\mathbb{R})$ and $\left(f^{-1}\right)_{\xi}-1 \in L^{2}(\mathbb{R})$. Hence $f, f^{-1} \in G$.

The lower bound on $y_{\xi}(\xi, t)+H_{\xi}(\xi, t)$ is given by (2.26), while the upper bound is a result of (2.14) combined with $\left|y_{0, \xi}(\xi)\right|,\left|H_{0, \xi}(\xi)\right|$, and $\left|U_{0, \xi}(\xi)\right|$ all being less than or equal to 1.

The set of admissible initial data in Lagrangian coordinates $\mathcal{F}_{i}^{\alpha}$ corresponds to a set of admissible initial data $\mathcal{D}_{0}^{\alpha}=M\left(\mathcal{F}_{i}^{\alpha}\right)$ in Eulerian coordinates as already hinted before. We want to characterize the set $\mathcal{D}_{0}^{\alpha}$ in terms of conditions on the measures $\mu$ and $\nu$.

Proposition 3.11. Let $\mathcal{D}_{0}^{\alpha}=M\left(\mathcal{F}_{i}^{\alpha}\right)$. Then $(u, \rho, \nu, \mu) \in \mathcal{D}_{0}^{\alpha}$ if and only if

$$
\nu=\mu \text {. }
$$

Proof. Assume that $X \in \mathcal{F}_{i}^{\alpha}$ and let $(u, \rho, \nu, \mu)=M(X)$. Then combining Definition 2.2 and Definition 3.3 yields

$$
\nu=y_{\#}\left(H_{\xi} d \xi\right)=y_{\#}\left(V_{\xi} d \xi\right)=\mu .
$$

Let $(u, \rho, \mu, \nu) \in \mathcal{D}^{\alpha}$ such that $\mu=\nu$. Denoting $X=L((u, \rho, \nu, \mu))$, then we have $\frac{d \mu}{d \nu}=1$ and subsequently

$$
V(\xi)=\int_{-\infty}^{\xi} H_{\xi}(\eta) d \eta=H(\xi) .
$$

That is $X \in \mathcal{F}_{i}^{\alpha}$ and since $M \circ L=\operatorname{id}_{\mathcal{D}^{\alpha}}$ we have that $(u, \rho, \nu, \mu) \in \mathcal{D}_{0}^{\alpha}$.

The set up is complete, but before defining $\alpha$-dissipative solutions in Eulerian coordinates with the help of the mappings $L$ and $M$, a remark on the properties imposed on $\alpha$.

Remark 3.12. We assume, see (2.1), that either $\alpha: \mathbb{R} \rightarrow[0,1)$ or $\alpha: \mathbb{R} \rightarrow 1$ instead of $\alpha: \mathbb{R} \rightarrow[0,1]$. The reason for this restriction is that in the latter case there exist solutions in Lagrangian coordinates for which Proposition 3.8 is not satisfies at all times. This is the case if there exist $t^{\star} \in[0, \infty)$ and $\xi_{1}, \xi_{2}, \xi_{3} \in \mathbb{R}$ with $\xi_{1}<\xi_{2}<\xi_{3}$ such that

- $y_{\xi}\left(\xi, t^{\star}\right)=0$ for all $\xi \in\left(\xi_{1}, \xi_{2}\right)$,

- $V_{\xi}\left(\xi, t^{\star}\right)=0$ for all $\xi \in\left(\xi_{1}, \xi_{3}\right)$,

- $V_{\xi}\left(\xi, t^{\star}\right) \neq 0$ for all $\xi \in\left(\xi_{3}, \xi_{2}\right)$,

as illustrated in Example A.4.

We give the definition of $\alpha$-dissipative solutions of (1.1). 
Definition 3.13. We say that $(u, \rho, \nu, \mu)$ is a weak solution of (1.1) with initial data $\left(u_{0}, \rho_{0}, \nu_{0}, \mu_{0}\right) \in \mathcal{D}_{0}^{\alpha}$ if

$$
\begin{aligned}
u & \in C^{0, \frac{1}{2}}(\mathbb{R} \times[0, T], \mathbb{R}), \quad \text { for any finite } T \geq 0, \\
\rho d x & \in C_{\text {weak } *}([0, \infty), \mathcal{M}(\mathbb{R})), \\
\nu & \in C_{\text {weak } *}\left([0, \infty), \mathcal{M}^{+}(\mathbb{R})\right), \\
(u(t), \rho(t), \nu(t), \mu(t)) & \in \mathcal{D}^{\alpha} \quad \text { for all } t \geq 0, \\
\left.(u, \rho, \nu, \mu)\right|_{t=0} & =\left(u_{0}, \rho_{0}, \nu_{0}, \mu_{0}\right), \\
\nu(t)(\mathbb{R}) & =\nu_{0}(\mathbb{R}) \quad \text { for all } t \geq 0,
\end{aligned}
$$

for each compactly supported test function $\phi \in C_{0}^{\infty}(\mathbb{R} \times[0, \infty))$,

$$
\begin{aligned}
\int_{0}^{\infty} \int_{\mathbb{R}}\left(u \phi_{t}+\frac{1}{2} u^{2} \phi_{x}+\frac{1}{4}\left(\int_{-\infty}^{x} d \mu-\int_{x}^{\infty} d \mu\right) \phi\right) d x d t & =-\left.\int_{\mathbb{R}} u_{0} \phi\right|_{t=0} d x \\
\int_{0}^{\infty} \int_{\mathbb{R}} \rho \phi_{t}+(\rho u) \phi_{x} d x d t & =-\left.\int_{\mathbb{R}} \rho_{0} \phi\right|_{t=0} d x
\end{aligned}
$$

and for each non-negative compactly supported test function $\phi \in C_{0}^{\infty}(\mathbb{R} \times[0, \infty))$,

$$
\int_{0}^{\infty} \int_{\mathbb{R}}\left(\phi_{t}+u \phi_{x}\right) d \mu(t) d t \geq-\left.\int_{\mathbb{R}} \phi\right|_{t=0} d \mu_{0} .
$$

If in addition for each $t \geq 0$ we have

$$
\begin{aligned}
d \mu(t) & =d \mu(t)_{a c}^{-}+(1-\alpha(x)) d \mu(x, t)_{s}^{-}, \\
\mu(s) & \stackrel{*}{\rightarrow} \mu(t) \text { as } s \downarrow t, \\
\mu(s) & \stackrel{*}{\rightarrow} \mu(t)^{-} \text {as } s \uparrow t,
\end{aligned}
$$

we call $t \mapsto(u(t), \rho(t), \nu(t), \mu(t))$ an $\alpha$-dissipative weak solution.

Now, $\alpha$-dissipative solutions can be constructed. The solution operator $T_{t}$ : $\mathcal{D}_{0}^{\alpha} \times[0, \infty) \rightarrow \mathcal{D}^{\alpha}$ is then given by $T_{t}=M \circ S_{t} \circ L$.

Theorem 3.14. To any initial data $\left(u_{0}, \rho_{0}, \nu_{0}, \mu_{0}\right) \in \mathcal{D}_{0}^{\alpha}$ there exists a globally defined $\alpha$-dissipative solution, $(u(t), \rho(t), \nu(t), \mu(t))$, in the sense of Definition 3.13 such that $(u(0), \rho(0), \nu(0), \mu(0))=\left(u_{0}, \rho_{0}, \nu_{0}, \mu_{0}\right)$.

Proof. First we show that the operator $T_{t}=M \circ S_{t} \circ L$ is indeed a solution operator on $\mathcal{D}^{\alpha}$. Let $(u(t), \rho(t), \nu(t), \mu(t))=T_{t}\left(\left(u_{0}, \rho_{0}, \nu_{0}, \mu_{0}\right)\right)$. Then we have $(u(t), \rho(t), \nu(t), \mu(t)) \in \mathcal{D}^{\alpha}$, and (3.18d)-(3.18f) are satisfied.

To show (3.19), we apply a change of variables in the integrals by letting $x=$ $y(\xi, t)$ for each $t$. Strictly speaking we have to integrate over the set $\{\xi \in \mathbb{R} \mid$ $\left.y_{\xi}(\xi, t)>0\right\}$, but since $y_{\xi}(\xi, t)=0$ implies that both $r(\xi, t)=0$ and $U_{\xi}(\xi, t)=0$ the integrands vanish on the complement of this set. We have that

$$
\begin{aligned}
& \int_{0}^{\infty} \int_{\mathbb{R}}\left(u \phi_{t}+\frac{1}{2} u^{2} \phi_{x}+\frac{1}{4}\left(\int_{-\infty}^{x} d \mu-\int_{x}^{\infty} d \mu\right) \phi\right) d x d t \\
& =\int_{0}^{\infty} \int_{\mathbb{R}} U\left(\phi_{t} \circ y+\frac{1}{2} U \phi_{x} \circ y\right) y_{\xi}(\xi) d \xi d t \\
& \quad+\frac{1}{4} \int_{0}^{\infty} \int_{\mathbb{R}}\left(\int_{-\infty}^{y(\xi)} d \mu-\int_{y(\xi)}^{\infty} d \mu\right) \phi \circ y y_{\xi} d \xi d t
\end{aligned}
$$




$$
\begin{aligned}
= & \int_{0}^{\infty} \int_{\mathbb{R}}\left[U(\xi, t)\left(\frac{d}{d t} \phi(y(\xi, t), t)-\frac{1}{2} U \phi_{x}(y(\xi, t), t)\right)\right. \\
& \left.+\frac{1}{2}\left(\int_{-\infty}^{\xi} V_{\xi}(\eta, t) d \eta-\frac{1}{2} \int_{-\infty}^{\infty} V_{\xi}(\eta, t) d \eta\right) \phi \circ y\right] y_{\xi} d \xi d t \\
= & \int_{0}^{\infty} \int_{\mathbb{R}}\left[\frac{d}{d t}(U \phi \circ y)-\frac{1}{2} U^{2} \phi_{x} \circ y\right] y_{\xi} d \xi d t \\
= & \int_{0}^{\infty} \int_{\mathbb{R}}\left[\frac{d}{d t}\left(U \phi \circ y y_{\xi}\right)-\frac{1}{2} U^{2} \phi_{x} \circ y y_{\xi}-U \phi \circ y y_{t, \xi}\right] d \xi d t \\
= & \int_{0}^{\infty} \int_{\mathbb{R}}\left[\frac{d}{d t}\left(U \phi \circ y y_{\xi}\right)-\left(\frac{1}{2} U^{2} \phi \circ y\right)_{\xi}\right] d \xi d t \\
= & -\left.\int_{\mathbb{R}} u_{0} \phi\right|_{t=0} d x .
\end{aligned}
$$

The equation for $\rho$ is treated in the same way,

$$
\begin{aligned}
\int_{0}^{\infty} \int_{\mathbb{R}} \rho \phi_{t}+(\rho u) \phi_{x} d x d t & =\int_{0}^{\infty} \int_{\mathbb{R}}\left(\phi_{t}+u \phi_{x}\right) \rho d x d t \\
& =\int_{0}^{\infty} \int_{\mathbb{R}}\left(\phi_{t} \circ y+U \phi_{x} \circ y\right) r d \xi d t \\
& =\int_{0}^{\infty} \int_{\mathbb{R}} \frac{d}{d t} \phi(y(\xi, t), t) r(\xi, t) d \xi d t \\
& =\int_{0}^{\infty} \int_{\mathbb{R}} \frac{d}{d t}(\phi(y(\xi, t), t) r(\xi, t)) d \xi d t \\
& =-\int_{\mathbb{R}} \phi(y(\xi, 0), 0) r(\xi, 0) d \xi \\
& =-\int_{\mathbb{R}} \rho_{0}(x) \phi(x, 0) d x .
\end{aligned}
$$

The inequality for $\mu$ is proved following the same lines

$$
\begin{aligned}
\int_{0}^{\infty} \int_{\mathbb{R}}\left(\phi_{t}+u \phi_{x}\right) d \mu(t) d t= & \int_{0}^{\infty} \int_{\mathbb{R}}\left(\phi_{t}+u \phi_{x}\right) \circ y V_{\xi} d \xi d t \\
= & \int_{0}^{\infty} \int_{\mathbb{R}} V_{\xi} \frac{d}{d t}(\phi \circ y) d \xi d t \\
= & \int_{\mathbb{R}}\left(\int_{0}^{\tau} H_{0, \xi} \frac{d}{d t}(\phi \circ y) d t\right. \\
& \left.\quad+\int_{\tau}^{\infty}(1-\alpha(y(\tau))) H_{0, \xi} \frac{d}{d t}(\phi \circ y) d t\right) d \xi \\
= & \int_{\mathbb{R}}\left(\left.\alpha(y(\tau)) H_{0, \xi} \phi \circ y\right|_{t=\tau}-\left.H_{0, \xi}(\phi \circ y)\right|_{t=0}\right) d \xi \\
\geq & -\left.\int_{\mathbb{R}} H_{0, \xi}(\phi \circ y)\right|_{t=0} d \xi \\
= & -\left.\int_{\mathbb{R}} \phi\right|_{t=0} d \mu_{0} .
\end{aligned}
$$

We are going to show that (3.18a - (3.18c) hold. First we show that $u$ is Hölder continuous. To that end let $\left(x_{1}, t_{1}\right),\left(x_{2}, t_{2}\right) \in \mathbb{R} \times[0, \infty)$. Then there exist $\xi_{1}$ and 
$\xi_{2} \in \mathbb{R}$ such that $y\left(\xi_{1}, t_{1}\right)=x_{1}$ and $y\left(\xi_{2}, t_{2}\right)=x_{2}$. Thus

$$
\begin{aligned}
\left|u\left(x_{1}, t_{1}\right)-u\left(x_{2}, t_{2}\right)\right|= & \left|u\left(y\left(\xi_{1}, t_{1}\right), t_{1}\right)-u\left(y\left(\xi_{2}, t_{2}\right), t_{2}\right)\right| \\
\leq & \left|\int_{y\left(\xi_{1}, t_{1}\right)}^{y\left(\xi_{2}, t_{1}\right)} u_{x}\left(x, t_{1}\right) d x\right|+\left|u\left(y\left(\xi_{2}, t_{1}\right), t_{1}\right)-u\left(y\left(\xi_{2}, t_{2}\right), t_{2}\right)\right| \\
\leq & \left\|u_{x}\left(\cdot, t_{1}\right)\right\|_{2} \sqrt{\left|y\left(\xi_{2}, t_{1}\right)-y\left(\xi_{1}, t_{1}\right)\right|}+\left|\int_{t_{1}}^{t_{2}} U_{t}\left(\xi_{2}, t\right) d t\right| \\
\leq & \sqrt{\nu_{0}(\mathbb{R})} \sqrt{\left|x_{2}-x_{1}\right|+\left|y\left(\xi_{2}, t_{2}\right)-y\left(\xi_{2}, t_{1}\right)\right|} \\
& +\frac{1}{4} \nu_{0}(\mathbb{R})\left|t_{2}-t_{1}\right| \\
\leq & \sqrt{\nu_{0}(\mathbb{R})} \sqrt{\left|x_{2}-x_{1}\right|+|| u_{0} \|_{\infty}\left|t_{2}-t_{1}\right|+\frac{1}{8} \nu_{0}(\mathbb{R})\left|t_{2}-t_{1}\right|^{2}} \\
& +\frac{1}{4} \nu_{0}(\mathbb{R})\left|t_{2}-t_{1}\right|,
\end{aligned}
$$

and thus $u$ is Hölder continuous. Moreover, if $t_{1}, t_{2} \leq T$ for some finite $T$, then

$$
\left|u\left(x_{1}, t_{1}\right)-u\left(x_{2}, t_{2}\right)\right| \leq C(1+\sqrt{T}) \sqrt{\left|x_{2}-x_{1}\right|+\left|t_{2}-t_{1}\right|},
$$

where $C$ depends on $\left\|u_{0}\right\|_{\infty}$ and $\nu_{0}(\mathbb{R})$ only. Hence $u$ is locally Hölder continuous both with respect to time and space with Hölder exponent one half.

Let $t \geq 0$ be given, and choose a sequence $t_{n}$ in $[0, \infty)$ that converges to $t$. In order to show the continuity of the measure $\nu$ let $\phi \in C_{c}^{\infty}(\mathbb{R})$ be given. Then

$$
\int_{\mathbb{R}} \phi(x) d \nu\left(t_{n}\right)=\int_{\mathbb{R}} \phi\left(y\left(\xi, t_{n}\right)\right) H_{0, \xi}(\xi) d \xi
$$

Since $\phi$ is bounded and of compact support, and $y\left(\xi, t_{n}\right)$ tends to $y(\xi, t)$ for almost every $\xi$ as $t_{n}$ tends to $t$, we have that $\phi\left(y\left(\xi, t_{n}\right)\right) H_{0, \xi}(\xi) \leq\|\phi\|_{\infty} H_{0, \xi}(\xi)$. Thus by the Lebesgue dominated convergence theorem $\nu\left(t_{n}\right)$ converges star weakly to $\nu(t)$ in the sense of measures.

The weak star continuity of $\rho d x$ is proved in the same manner as for $\nu$.

Next, we show that (3.20) holds. The proof is similar to the one of [12, Theorem 4.3]. Let $t>0$ be given. Then we have that

$$
\begin{aligned}
& \lim _{s \uparrow t} V_{\xi}(\xi, s)= \begin{cases}H_{0, \xi}(\xi), & \text { if } t \leq \tau(\xi), \\
V_{\xi}(\xi, t), & \text { if } \tau(\xi)<t .\end{cases} \\
& \lim _{s \downarrow t} V_{\xi}(\xi, s)=V_{\xi}(\xi, t) .
\end{aligned}
$$

We want to prove that

$$
\begin{aligned}
& \lim _{s \uparrow t} \mu(s)=y(t)_{\#}\left(\lim _{s \uparrow t} V_{\xi}(\xi, s) d \xi\right), \\
& \lim _{s \downarrow t} \mu(s)=y(t)_{\#}\left(V_{\xi}(\xi, s) d \xi\right)=\mu(t),
\end{aligned}
$$

where the limit is in the weak star sense in $\mathcal{M}^{+}(\mathbb{R})$. Let $\phi \in C_{c}^{\infty}(\mathbb{R})$ be given, then

$$
\int_{\mathbb{R}} \phi(x) d \mu(s)=\int_{\mathbb{R}} \phi(y(\xi, s)) V_{\xi}(\xi, s) d \xi .
$$

Since $\phi$ is bounded and of compact support, and $y(\xi, s)$ tends to $y(\xi, t)$ for almost every $\xi$ as $s$ tends to $t$, we have that $\phi(y(\xi, s)) V_{\xi}(\xi, s) \leq\|\phi\|_{\infty} H_{0, \xi}(\xi)$. Thus by 
the Lebesgue dominated convergence theorem (3.29) hold. Let us prove that

$$
d \mu(t)=d \mu(t)_{a c}^{-}+(1-\alpha(x)) d \mu(t)_{s}^{-},
$$

where $\mu(t)^{-}=\lim _{s \uparrow t} \mu(s)$. We have that

$$
\begin{aligned}
\mu(t)^{-}-\mu(t) & =y(t)_{\#}\left(\lim _{s \uparrow t} V_{\xi}(\xi, s) d \xi\right)-y(t)_{\#}\left(V_{\xi}(\xi, t) d \xi\right) \\
& =y(t)_{\#}\left(\left(\lim _{s \uparrow t} V_{\xi}(\xi, s)-V_{\xi}(\xi, t)\right) d \xi\right) \\
& =y(t)_{\#}\left(\alpha(y(\xi, t)) H_{0, \xi}(\xi) \mathbf{1}_{\left\{\xi \mid y_{\xi}(\xi, t)=0\right\}} d \xi\right) .
\end{aligned}
$$

Define for each $t$ the sets

$$
\begin{aligned}
& B(t)=\left\{\xi \mid y_{\xi}(\xi, t)>0\right\} \\
& A(t)=y(B, t) .
\end{aligned}
$$

Let us show that $A(t)$ is of full measure. Since $y(\cdot, t)$ is surjective $A(t)^{c} \subseteq y\left(B(t)^{c}, t\right)$, and thus

$$
\mathrm{m}\left(A(t)^{c}\right) \leq \int_{y\left(B(t)^{c}, t\right)} d \xi=\int_{B(t)^{c}} y_{\xi}(\xi, t) d \xi=0 .
$$

We prove that $y_{\xi}(\xi, t)>0$ almost everywhere in $y(t)^{-1}(A(t))$. Assume that there exists $\xi \in y(t)^{-1}(A(t))$ such that $y_{\xi}(\xi, t)=0$. Then $\xi \in B(t)^{c}$, and there must be $\xi^{\prime}$ in $B(t)$ such that $y\left(\xi^{\prime}, t\right)=y(\xi, t)$. But then $\left[\xi, \xi^{\prime}\right] \subseteq y(t)^{-1}(A(t))$, and $y(\eta, t)=y\left(\xi^{\prime}, t\right)$ for $\eta \in\left(\xi, \xi^{\prime}\right)$. Thus either $y_{\xi}\left(\xi^{\prime}, t\right)=0$ or $y_{\xi}\left(\xi^{\prime}, t\right)$ is undefined, which contradicts $\xi^{\prime} \in B(t)$.

Equation (3.32) implies that on any measurable set $C \subseteq A(t)$ the measure $\mu(t)^{-}-$ $\mu(t)$ is zero. Hence $\mu(t)^{-}-\mu(t)$ is supported on a set of measure zero, and is thus singular with respect to the Lebesgue measure. By the general change of variable formula for any $C \subseteq A(t)^{c}$ we have that $\left(\mu(t)^{-}-\mu(t)\right)(C)=\int_{C} \alpha(x) d \mu(t)^{-}$, and hence $\left(\mu(t)^{-}-\mu(t)\right)=\alpha d\left(\left.\mu(t)^{-}\right|_{A(t)^{c}}\right)$. To complete the proof we need to prove that

$$
\mu(t)_{a c}^{-}=\left.\mu(t)^{-}\right|_{A(t)},
$$

which would imply that $\mu(t)_{s}^{-}=\left.\mu(t)^{-}\right|_{A(t)^{c}}$. First we note that $\mathrm{m}\left(A(t)^{c}\right)=0$. Thus for any measurable $C$

$$
\begin{aligned}
\mu(t)_{a c}^{-}(C) & \leq \mu(t)_{a c}^{-}(C \cap A(t))+\mu(t)_{a c}^{-}\left(C \cap A(t)^{c}\right) \\
& \leq \mu(t)^{-}(C \cap A(t)),
\end{aligned}
$$

and hence $\mu(t)_{a c}^{-} \leq\left.\mu(t)^{-}\right|_{A(t)}$. We must show that $\left.\mu(t)^{-}\right|_{A(t)}$ is absolutely continuous. Let $E$ be a set of measure zero and define $K_{M}=\left\{\xi \in \mathbb{R} \mid \frac{V_{\xi}(\xi, t)}{y_{\xi}(\xi, t)} \leq M\right\}$. Then $\mathbf{1}_{K_{M}}$ tends pointwise to one in $y(t)^{-1}(A(t))$, and thus

$$
\begin{aligned}
\int_{y^{-1}(A(t) \cap E)} V_{\xi}(\xi, t) \mathbf{1}_{K_{M}} d \xi & \leq M \int_{y^{-1}(A(t) \cap E)} y_{\xi}(\xi, t) d \xi \\
& =M \mathrm{~m}(A(t) \cap E) \\
& =0 .
\end{aligned}
$$

Hence by the monotone convergence theorem $\left.\mu(t)^{-}\right|_{A(t)}(E)=0$ for all sets $E$ of measure zero, and $\left.\mu(t)^{-}\right|_{A(t)}$ is absolutely continuous. 
See Example A.1 for an example of an $\alpha$-dissipative solution in Eulerian coordinates.

We end this section by a corollary that connects the notion of $\alpha$-dissipative solutions to the construction of dissipative solutions by Bressan and Constantin [1], Dafermos [7, Zhang and Zheng [19, and Wunsch [18.

Corollary 3.15. In the special case $\alpha(x)$ being constant, $\nu_{0}=\mu_{0}=\mu_{0, a c}$, and $\rho_{0} \equiv$ 0 there exists a formula for the solution similar to the formula in [7, Theorem 2.1] for $\alpha \equiv 1$. More precisely, choosing as initial characteristic the identity function, i.e. $y(\xi, 0)=\xi$ (which is always possible in this case), one obtains

$$
\begin{gathered}
u(x, t)=u_{0}\left(\xi_{x, t}\right)+\frac{1}{2} \int_{0}^{t}\left(\int_{-\infty}^{\xi_{x, t}}\left(1-\alpha \mathbf{1}_{\left\{z \mid 0 \leq \frac{-2}{u_{0, x}(z)} \leq s\right\}}\right) u_{0, x}(z)^{2} d z\right. \\
\left.(3.39) \quad-\frac{1}{2} \int_{-\infty}^{\infty}\left(1-\alpha \mathbf{1}_{\left\{z \mid 0 \leq \frac{-2}{u_{0, x}(z)} \leq s\right\}}\right) u_{0, x}(z)^{2} d z\right) d s \\
x=\xi_{x, t}+u_{0}\left(\xi_{x, t}\right) t+\frac{1}{2} \int_{0}^{t} \int_{0}^{s}\left(\int_{-\infty}^{\xi_{x, t}}\left(1-\alpha \mathbf{1}_{\left\{z \mid 0 \leq \frac{-2}{u_{0, x}(z)} \leq \sigma\right\}}\right) u_{0, x}(z)^{2} d z\right. \\
\left.\quad-\frac{1}{2} \int_{-\infty}^{\infty}\left(1-\alpha \mathbf{1}_{\left\{z \mid 0 \leq \frac{-2}{u_{0, x}(z)} \leq \sigma\right\}}\right) u_{0, x}(z)^{2} d z\right) d \sigma d s,
\end{gathered}
$$

where $\xi_{x, t}$ is given implicitely through the relation $x=y\left(\xi_{x, t}, t\right)$.

\section{The LipschitZ STABILITY}

In this section we construct a parametrized family of metrics on bounded sets of $\mathcal{D}^{\alpha}$ that renders the flow Lipschitz continuous with respect to the initial data. The construction is based on the ones in 4, 10, 11. The idea is to first create a parametrized family of metrics $\tilde{d}(t, \cdot, \cdot)$ on $\mathcal{F}^{\alpha}$, and then to use $\tilde{d}(t, \cdot, \cdot)$ to construct a parametrized family of metrics on $\mathcal{D}^{\alpha}$.

We start by motivating the choice of the set of initial data $\mathcal{D}_{0}^{\alpha}$. Having a close look at the construction of $\alpha$-dissipative solutions in the last two sections, it is natural to require that the initial data $\left(u_{0}, \rho_{0}, \mu_{0}, \nu_{0}\right) \in \mathcal{D}_{0}^{\alpha}$. To be more precise, all the important information about the solution is contained in the functions $(u, \rho, \mu)$ while the measure $\nu$ is only added for technical reasons. Correspondingly the important information in Lagrangian coordinates is encoded in the functions $(y, U, r, V)$ while $H$ is a help function. In particular, one has that $M((y, U, H, r, V))$ and $M((y, U, \tilde{H}, r, V))$ yield the same triplet $(u, \rho, \mu)$ in Eulerian coordinates. Thus if we do not have initial data in $\mathcal{D}_{0}^{\alpha}$ we can get solutions which are equal for all practical purposes but far apart in Lagrangian coordinates. In addition, this choice is consistent with the conservative solutions constructed in [16], that is $\alpha \equiv 0$, since $\nu(t)=\mu(t)$ for all $t \geq 0$ for conservative solutions.

Beside of the choice of the initial data, there is one more major difficulty. As illustrated in Example A.2, solutions do not necessarily play nicely with the $B$ norm. In particular, the sudden changes in the energy can lead to jumps in the distance. To model the drop in the energy at wave breaking in a continuous way we introduce a function $g$, which separates points where wave breaking will occur and points where there will be no wave breaking. We start by splitting $\mathbb{R}$ into two regions: One consisting of all points $\xi \in \mathbb{R}$ where wave breaking will occur and one where there will be no wave breaking. 
Definition 4.1. For each $X \in \mathcal{F}^{\alpha}$ define the sets $\Omega_{c}(X)$, and $\Omega_{d}(X)$ by

$$
\begin{aligned}
& \Omega_{c}(X)=\left\{\xi \in \mathbb{R} \mid U_{\xi}(\xi) \geq 0 \text { or } r(\xi) \neq 0\right\}, \\
& \Omega_{d}(X)=\left\{\xi \in \mathbb{R} \mid U_{\xi}(\xi)<0 \text { and } r(\xi)=0\right\} .
\end{aligned}
$$

For each $X \in \mathcal{F}^{\alpha}$ the real line splits into mutually disjoint parts $\Omega_{\iota}(X), \iota=c, d$. Given $X_{0} \in \mathcal{F}_{i}^{\alpha}$ and $\xi \in \mathbb{R}$, observe that if you start initially in $\Omega_{c}\left(X_{0}\right), c$ for conservative (or continuous), you will remain there for all later times. That is, $\Omega_{c}\left(X_{0}\right) \subseteq \Omega_{c}(X(t))$ for all $t \geq 0$. If you, on the other hand, start initially in $\Omega_{d}\left(X_{0}\right), d$ for dissipative (or discontinuous), you will at the wave breaking time $\tau$, given by (2.16), enter $\Omega_{c}(X(\tau))$. In other words, $\cap_{t \geq 0} \Omega_{d}(X(t))=\emptyset$.

To model continuously the drop in the energy we introduce the function $g$ and for technical reasons in addition $g_{2}$ and $g_{3}$.

Definition 4.2. For each $X \in \mathcal{F}^{\alpha}$ and $\xi \in \mathbb{R}$ define

$$
g(X(\xi))= \begin{cases}y_{\xi}(\xi)+H_{\xi}(\xi)-\alpha(y(\xi)) H_{\xi}(\xi), & \xi \in \Omega_{d}(X), \\ y_{\xi}(\xi)+V_{\xi}(\xi), & \xi \in \Omega_{c}(X) .\end{cases}
$$

Furthermore define $g_{2}$ by

$$
g_{2}(X(\xi))= \begin{cases}\left\|\alpha^{\prime}\right\|_{\infty} H_{\infty} U_{\xi}(\xi), & \xi \in \Omega_{d}(X), \\ 0, & \xi \in \Omega_{c}(X) .\end{cases}
$$

and $g_{3}$ by

$$
g_{3}(X(\xi))= \begin{cases}\left\|\alpha^{\prime}\right\|_{\infty} U(\xi) U_{\xi}(\xi), & \xi \in \Omega_{d}(X), \\ 0, & \xi \in \Omega_{c}(X),\end{cases}
$$

where $H_{\infty}=\lim _{\xi \rightarrow \infty} H(\xi)$.

By construction we have that $g(X)-1, g_{2}(X)$, and $g_{3}(X) \in L^{2}(\mathbb{R})$. We want to show that $t \mapsto g(X(t)), t \mapsto g_{2}(X(t)), t \mapsto g_{3}(X(t))$ are well-defined functions, which are continuous in time, for any solution $X(t)=S_{t}\left(X_{0}\right)$.

Proposition 4.3. Let $X(\xi, t)=S_{t}\left(X_{0}(\xi)\right)$ for some $X_{0} \in \mathcal{F}_{i}^{\alpha}$ and $\xi \in \mathbb{R}$. Then, the mappings $t \mapsto g(X(\xi, t))-1, t \mapsto g_{2}(X(\xi, t)), t \mapsto g_{3}(X(\xi, t))$ are continuous with respect to the $L^{2}(\mathbb{R})$-norm.

Proof. For $t \neq \tau(\xi)$ the functions are pointwise continuous since for $\xi$ given $U, U_{\xi}$, $y_{\xi}, H_{\xi}, V_{\xi}, y$ and $\alpha$ are continuous functions when $t \neq \tau(\xi)$. What remains is to prove that they are pointwise continuous at $t=\tau(\xi)$. We look at $g$ first. By (2.9) and (2.15),

$$
\begin{aligned}
\lim _{t \uparrow \tau(\xi)} g(X(\xi, t)) & =\lim _{t \uparrow \tau(\xi)}\left(y_{\xi}(\xi, t)+(1-\alpha(y(\xi, t))) H_{0, \xi}(\xi)\right) \\
& =y_{\xi}(\xi, \tau(\xi))+(1-\alpha(y(\xi, \tau(\xi)))) H_{0, \xi}(\xi) \\
& =y_{\xi}(\xi, \tau(\xi))+V_{\xi}(\xi, \tau(\xi)) \\
& =\lim _{t \downarrow \tau(\xi)}\left[y_{\xi}(\xi, t)+V_{\xi}(\xi, t)\right] .
\end{aligned}
$$

From (2.9) we have that $U_{\xi}(\xi, t) \rightarrow 0$ as $t \rightarrow \tau(\xi)$, and hence $t \mapsto g_{2}(X(\xi, t))$ and $t \mapsto g_{3}(X(\xi, t))$ are continuous at $t=\tau(\xi)$. 
Since $g\left(X\left(\xi, t^{\prime}\right)\right) \rightarrow g(X(\xi, t))$ pointwise as $t^{\prime}$ tends to $t$, we have by the dominated convergence theorem

$$
\lim _{t^{\prime} \rightarrow t} \int_{\mathbb{R}}\left(g\left(X\left(\xi, t^{\prime}\right)\right)-g(X(\xi, t))\right)^{2} d \xi=\int_{\mathbb{R}} \lim _{t^{\prime} \rightarrow t}\left(g\left(X\left(\xi, t^{\prime}\right)\right)-g(X(\xi, t))\right)^{2} d \xi=0,
$$

if we can find a function $k(\xi) \in L^{1}(\mathbb{R})$ such that $\left|g\left(X\left(\xi, t^{\prime}\right)\right)-g(X(\xi, t))\right|^{2} \leq k(\xi)$ for a.e $\xi$. Therefore recall (2.14), which implies that

$$
\left|U_{\xi}(\xi, t)\right| \leq\left|U_{0, \xi}(\xi)\right|+\frac{1}{2} t H_{0, \xi}(\xi)
$$

and

$$
\left|y_{\xi}(\xi, t)-1\right| \leq\left|y_{0, \xi}(\xi)-1\right|+t\left|U_{0, \xi}(\xi)\right|+\frac{1}{4} t^{2} H_{0 \xi}(\xi) .
$$

Thus one possible choice for $k(\xi)$ is

$$
k(\xi)=\left(2\left|y_{0, \xi}(\xi)\right|+2 T\left|U_{0, \xi}(\xi)\right|+\left(2+\frac{1}{2} T^{2}\right) H_{0, \xi}(\xi)\right)^{2}
$$

where $T=2 t$. The same argumentation holds for $g_{2}$ and $g_{3}$.

To simplify the notation we make the following definition.

Definition 4.4. Given $X=(y, U, H, r, V) \in \mathcal{F}^{\alpha}$, define $Z=\left(y_{\xi}, U_{\xi}, H_{\xi}, r\right)$.

We start by introducing a natural, preliminary metric on $\mathcal{F}^{\alpha}$ which will form the basis for establishing the Lipschitz stability later on.

Definition 4.5. Let $\tilde{d}: \mathcal{F}^{\alpha} \times \mathcal{F}^{\alpha} \rightarrow[0, \infty)$ be defined by

$$
\begin{aligned}
\tilde{d}(X, \bar{X})= & \|y-\bar{y}\|_{\infty}+\|U-\bar{U}\|_{\infty}+\left\|\alpha^{\prime}\right\|_{\infty}\left\|U H_{\xi}-\bar{U} \bar{H}_{\xi}\right\|_{2} \\
& +\left\|H_{\xi}-\bar{H}_{\xi}\right\|_{1}+\|Z-\bar{Z}\|_{2}+\|g(X)-g(\bar{X})\|_{2} \\
& +\left\|g_{2}(X)-g_{2}(\bar{X})\right\|_{2}+\left\|g_{3}(X)-g_{3}(\bar{X})\right\|_{2}
\end{aligned}
$$

Note that $\tilde{d}$ defines a metric on $\mathcal{F}^{\alpha}$ since $g, g_{2}$, and $g_{3}$ are well defined. The metric $\tilde{d}$ will turn out to be a Lipschitz continuous with respect to time for carefully selected initial data in $\mathcal{F}^{\alpha}$. The Lipschitz constant will depend on the total energy of the initial data and hence we will have to restrict our attention to subsets of $\mathcal{F}^{\alpha}$ whose elements have bounded total energy.

Definition 4.6. Let $\mathcal{F}^{\alpha, M}$ and $\mathcal{F}_{0}^{\alpha, M}$ be the following closed subsets of $\mathcal{F}^{\alpha}$

$$
\begin{aligned}
& \mathcal{F}^{\alpha, M}=\left\{X \in \mathcal{F}^{\alpha} \mid\|H\|_{\infty} \leq M\right\}, \\
& \mathcal{F}_{0}^{\alpha, M}=\mathcal{F}_{0}^{\alpha} \cap \mathcal{F}^{\alpha, M} .
\end{aligned}
$$

Correspondingly, denote by $\mathcal{D}^{\alpha, M}$ the subset of $\mathcal{D}^{\alpha}$ given by,

$$
\mathcal{D}^{\alpha, M}=\left\{(u, \rho, \nu, \mu) \in \mathcal{D}^{\alpha} \mid \nu(\mathbb{R}) \leq M\right\} .
$$

Note that the mappings $M$ and $L$ respect the energy bound,

$$
\begin{aligned}
L\left(\mathcal{D}^{\alpha, M}\right) & =\mathcal{F}_{0}^{\alpha, M}, \\
M\left(\mathcal{F}^{\alpha, M}\right) & =\mathcal{D}^{\alpha, M} .
\end{aligned}
$$

The construction $\tilde{d}$ might seem arbitrary, but it is connected to Eulerian coordinates as shown in the following remark. 
Remark 4.7. The terms in the definition of $\tilde{d}$ are natural in the sense that for smooth $(u, \rho, \nu, \mu)$ the various terms can be translated to Eulerian coordinates. In particular for $x=y(\xi)$ we have

$$
\begin{aligned}
y_{\xi}(\xi) & =\frac{1}{1+u_{x}(x)^{2}+\rho(x)^{2}}, \\
H_{\xi}(\xi) & =\frac{u_{x}(x)^{2}+\rho(x)^{2}}{1+u_{x}(x)^{2}+\rho(x)^{2}}, \\
U_{\xi}(\xi) & =\frac{u_{x}(x)}{1+u_{x}(x)^{2}+\rho(x)^{2}}, \\
r(\xi) & =\frac{\rho(x)}{1+u_{x}(x)^{2}+\rho(x)^{2}},
\end{aligned}
$$

and thus

$$
\begin{gathered}
U(\xi) H_{\xi}(\xi)=u(x) \frac{u_{x}(x)^{2}+\rho(x)^{2}}{1+u_{x}(x)^{2}+\rho(x)^{2}}, \\
\alpha(y(\xi)) H_{\xi}=\alpha(x) \frac{u_{x}(x)^{2}+\rho(x)^{2}}{1+u_{x}(x)^{2}+\rho(x)^{2}}, \\
\left\|\alpha^{\prime}\right\|_{\infty} H_{\infty} U_{\xi}(\xi)=\left\|\alpha^{\prime}\right\|_{\infty} \nu(\mathbb{R}) \frac{u_{x}(x)}{1+u_{x}(x)^{2}+\rho(x)^{2}}, \\
\left\|\alpha^{\prime}\right\|_{\infty} U(\xi) U_{\xi}(\xi)=\left\|\alpha^{\prime}\right\|_{\infty} u(x) \frac{u_{x}(x)}{1+u_{x}(x)^{2}+\rho(x)^{2}} .
\end{gathered}
$$

We will now construct a metric on $\mathcal{F}_{0}^{\alpha}$ that renders the flow Lipschitz continuous with respect to the initial data. The metric $\tilde{d}$ will unfortunately give a positive distance between $X$ and $X \bullet f$, even though they will map to the same element in Eulerian coordinates via the mapping $M$. Following [10, we minimize the distance over all possible relabelings and define $J: \mathcal{F}^{\alpha} \times \mathcal{F}^{\alpha} \rightarrow \mathbb{R}$ by

$$
J(X, \bar{X})=\inf _{f, g \in G}(\tilde{d}(X \bullet f, \bar{X})+\tilde{d}(X, \bar{X} \bullet g)) .
$$

Now, $J$ will not separate $X$ and $X \bullet f$. However $J$ is not a metric, as the triangle inequality fails. One can obtain a metric by summing over finite sequences in $\mathcal{F}_{0}^{\alpha, M}$ as follows.

Definition 4.8. Let $X, \bar{X} \in \mathcal{F}_{i, 0}^{\alpha, M}$, then define $d_{M}:[0, \infty) \times \mathcal{F}_{i, 0}^{\alpha, M} \times \mathcal{F}_{i, 0}^{\alpha, M} \rightarrow \mathbb{R}$ by

$$
d_{M}(t, X, \bar{X})=\inf \sum_{n=1}^{N} J\left(\Pi S_{t}\left(X_{n-1}\right), \Pi S_{t}\left(X_{n}\right)\right),
$$

where the infimum is taken over all finite sequences $\left\{X_{n}\right\}_{n=0}^{N}$ in $\mathcal{F}_{i, 0}^{\alpha, M}$ such that the endpoints $X_{0}$ and $X_{N}$ satisfy

$$
\begin{aligned}
& X=X_{0}, \\
& \bar{X}=X_{N} .
\end{aligned}
$$

It is not at all clear that $d_{M}(t, X, \bar{X})$ only vanishes when $X=\bar{X}$. The purpose of the next lemma is to assert that we have a positive lower bound on $d_{M}(t, X, \bar{X})$ when $X$ differs from $\bar{X}$. 
Lemma 4.9 ([10, Lemma 3.2]). Given $X, \bar{X} \in \mathcal{F}_{i, 0}^{\alpha, M}$ and $t \geq 0$, let $X_{1}(t)=$ $\Pi S_{t}(X)$ and $\bar{X}_{1}(t)=\Pi S_{t}(\bar{X})$, then

(4.19) $\left\|y_{1}(t)-\bar{y}_{1}(t)\right\|_{\infty}+\left\|U_{1}(t)-\bar{U}_{1}(t)\right\|_{\infty}+\left\|H_{1}(t)-\bar{H}_{1}(t)\right\|_{\infty} \leq 2 d_{M}(t, X, \bar{X})$.

Lemma 4.9 states that if the distance between $X_{1}(t)=\Pi S_{t}(X)$ and $\bar{X}_{1}(t)=$ $\Pi S_{t}(\bar{X})$ equals zero, then $\left(y_{1}, U_{1}, H_{1}\right)$ and $\left(\bar{y}_{1}, \bar{U}_{1}, \bar{H}_{1}\right)$ coincide. Still, $r_{1}$ and $\bar{r}_{1}$, and $V_{1}$ and $\bar{V}_{1}$ could, in principle, differ. The following lemmas shows that this cannot be the case, and consequently $d_{M}$ is a metric on $\Pi S_{t}\left(\mathcal{F}_{i, 0}^{\alpha, M}\right)$.

Lemma 4.10 ([11, A weaker form of Lemma 6.4]). Given $X, \bar{X}$ in $\mathcal{F}_{i, 0}^{\alpha, M}$ and $t \geq 0$, let $X_{1}(t)=\Pi S_{t}(X)$ and $\bar{X}_{1}(t)=\Pi S_{t}(\bar{X})$, then $d_{M}(t, X, \bar{X})=0$ implies that $r_{1}(t)=\bar{r}_{1}(t)$.

Lemma 4.11. Let $X_{0}, \bar{X}_{0} \in \mathcal{F}_{0}^{\alpha}$, and $X_{1}(t)=\Pi S_{t}\left(X_{0}\right), \bar{X}_{1}(t)=\Pi S_{t}\left(\bar{X}_{0}\right)$. If $d_{M}\left(t, X_{0}, \bar{X}_{0}\right)=0$ then $V_{1}(t)=\bar{V}_{1}(t)$.

Proof. From Lemma 4.9 and 4.10 we have that

$$
\left(y_{1}(t), U_{1}(t), H_{1}(t), r_{1}(t)\right)=\left(\bar{y}_{1}(t), \bar{U}_{1}(t), \bar{H}_{1}(t), \bar{r}_{1}(t)\right)
$$

and thus for almost all $\xi \in \mathbb{R}$

$$
\begin{aligned}
y_{1, \xi}(\xi, t) V_{1, \xi}(\xi, t) & =U_{1, \xi}(\xi, t)^{2}+r_{1}(\xi, t)^{2} \\
& =\bar{U}_{1, \xi}(\xi, t)^{2}+\bar{r}_{1}(\xi, t)^{2} \\
& =\bar{y}_{1, \xi}(\xi, t) \bar{V}_{1, \xi}(\xi, t) .
\end{aligned}
$$

Thus we have that $\bar{V}_{1, \xi}(t)=V_{1, \xi}(t)$ almost everywhere in the set $\left\{\xi \in \mathbb{R} \mid y_{1, \xi}(\xi, t)>\right.$ $0\}$. We turn our attention to the set $\left\{\xi \mid y_{1, \xi}(\xi, t)=0\right\}$. Assume that $\alpha=1$. Then $y_{1, \xi}(\xi, t)=0$ implies that $V_{1, \xi}(\xi, t)=0$ and thus $V_{1, \xi}(t)=\bar{V}_{1, \xi}(t)$ almost everywhere. Assume that $0 \leq \alpha<1$. Then if $y_{1, \xi}(\xi, t)=0$ we have that $t=\tau_{1}(\xi)$. There are two cases to consider. If $t=0$ we have by definition that $V_{1, \xi}(\xi, t)=H_{1, \xi}(\xi, t)=\bar{H}_{1, \xi}(\xi, t)=\bar{V}_{1, \xi}(\xi, t)$. If $t>0$, then we have from the time evolution operator $S_{t}$ that $V_{1, \xi}(\xi, t)=\left(1-\alpha\left(y_{1}\left(\xi, \tau_{1}(\xi)\right)\right)\right) H_{1, \xi}(\xi, t)=\bar{V}_{1, \xi}(\xi, t)$. Thus $V_{1, \xi}(\xi, t)=\bar{V}_{1, \xi}(\xi, t)$ for almost all $\xi \in \mathbb{R}$, and since $\lim _{\xi \rightarrow-\infty} V_{1}(\xi, t)=0=$ $\lim _{\xi \rightarrow-\infty} \bar{V}_{1}(\xi, t)$ we have that $V_{1}(\xi, t)=\bar{V}_{1}(\xi, t)$.

In order to estimate the time evolution of $d_{M}\left(t, X_{0}, \bar{X}_{0}\right)$, the following lemma is essential.

Lemma 4.12 ([16, Lemma 4.8]). If $X_{0}, \bar{X}_{0} \in \mathcal{F}_{i, 0}^{\alpha, M}$, then

$$
J\left(\Pi S_{t}\left(X_{0}\right), \Pi S_{t}\left(\bar{X}_{0}\right)\right) \leq e^{\frac{1}{2} t} J\left(S_{t}\left(X_{0}\right), S_{t}\left(\bar{X}_{0}\right)\right) .
$$

Thus we estimate the time evolution of $\tilde{d}\left(S_{t}\left(X_{0}\right), S_{t}\left(\bar{X}_{0}\right) \bullet f\right)$ for $X_{0}, \bar{X}_{0} \in \mathcal{F}_{i, 0}^{\alpha, M}$ and $f \in G$. First let us see how we can use the function $g$ to bound the time evolution of $\|Z(t)-\bar{Z}(t)\|_{2}$.

Lemma 4.13. Let $X(t)$ and $\bar{X}(t)$ be the solutions with initial data $X_{0}, \bar{X}_{0} \in \mathcal{F}_{i}^{\alpha}$, then

$$
\begin{aligned}
\left\|y_{\xi}(t)-\bar{y}_{\xi}(t)\right\|_{2} & \leq\left\|y_{0, \xi}-\bar{y}_{0, \xi}\right\|_{2}+\int_{0}^{t}\left\|U_{\xi}(s)-\bar{U}_{\xi}(s)\right\|_{2} d s \\
\left\|U_{\xi}(t)-\bar{U}_{\xi}(t)\right\|_{2} & \leq\left\|U_{0, \xi}-\bar{U}_{0, \xi}\right\|_{2}+\frac{1}{2} t\left\|H_{0, \xi}-\bar{H}_{0, \xi}\right\|_{2}
\end{aligned}
$$




$$
+\frac{1}{2} \int_{0}^{t}\left(\|g(X(s))-g(\bar{X}(s))\|_{2}+\left\|y_{\xi}(s)-\bar{y}_{\xi}(s)\right\|_{2}\right) d s,
$$

(4.23c) $\left\|H_{\xi}(t)-\bar{H}_{\xi}(t)\right\|_{2}=\left\|H_{0, \xi}-\bar{H}_{0, \xi}\right\|_{2}$,

$$
\|r(t)-\bar{r}(t)\|_{2}=\left\|r_{0}-\bar{r}_{0}\right\|_{2} \text {. }
$$

Proof. To write more concisely we omit $\xi$ from the notation in this proof. We have that for any $\xi \in \mathbb{R}$,

$$
\begin{aligned}
|r(t)-\bar{r}(t)| & =\left|r_{0}-\bar{r}_{0}\right|, \\
\left|H_{\xi}(t)-\bar{H}_{\xi}(t)\right| & =\left|H_{0, \xi}-\bar{H}_{0, \xi}\right|, \\
\left|y_{\xi}(t)-\bar{y}_{\xi}(t)\right| & \leq\left|y_{0, \xi}-\bar{y}_{0, \xi}\right|+\int_{0}^{t}\left|U_{\xi}(s)-\bar{U}_{\xi}(s)\right| d s .
\end{aligned}
$$

There are three cases to consider for the estimate of $\left|U_{\xi}(t)-\bar{U}_{\xi}(t)\right|$.

If $\xi \in \Omega_{c}\left(X_{0}\right) \cap \Omega_{c}\left(\bar{X}_{0}\right)$, then

$$
\left|U_{\xi}(t)-\bar{U}_{\xi}(t)\right| \leq\left|U_{0, \xi}-\bar{U}_{0, \xi}\right|+\frac{1}{2} \int_{0}^{t}\left|H_{0, \xi}-\bar{H}_{0, \xi}\right| d s .
$$

Assume that $\xi \in \Omega_{d}\left(X_{0}\right) \cap \Omega_{c}\left(\bar{X}_{0}\right)$. Then we have for $t<\tau$ that

$$
\left|U_{\xi}(t)-\bar{U}_{\xi}(t)\right| \leq\left|U_{0, \xi}-\bar{U}_{0, \xi}\right|+\frac{1}{2} \int_{0}^{t}\left|H_{0, \xi}-\bar{H}_{0, \xi}\right| d s .
$$

If $t \geq \tau$ we have

$$
\begin{aligned}
& \left|U_{\xi}(t)-\bar{U}_{\xi}(t)\right| \leq\left|U_{0, \xi}-\bar{U}_{0, \xi}\right|+\frac{1}{2} \int_{0}^{\tau}\left|V_{\xi}(s)-\bar{H}_{0, \xi}\right| d s+\frac{1}{2} \int_{\tau}^{t}\left|H_{0, \xi}-\bar{H}_{0, \xi}\right| d s \\
& \leq\left|U_{0, \xi}-\bar{U}_{0, \xi}\right|+\frac{1}{2} t\left|H_{0, \xi}-\bar{H}_{0, \xi}\right| \\
& \quad+\frac{1}{2} \int_{0}^{t}\left(|g(X(s))-g(\bar{X}(s))|+\left|y_{\xi}(s)-\bar{y}_{\xi}(s)\right|\right) d s .
\end{aligned}
$$

Let $\xi \in \Omega_{d}\left(X_{0}\right) \cap \Omega_{d}\left(\bar{X}_{0}\right)$. If $\tau \leq t<\bar{\tau}$, then $V_{\xi}(s) \leq H_{0, \xi}, U_{\xi}(t) \geq 0 \geq \bar{U}_{\xi}(t)$, and both $U_{\xi}(t), \bar{U}_{\xi}(t)$ are non-decreasing. Hence

$$
0 \leq U_{\xi}(t)-\bar{U}_{\xi}(t) \leq\left|U_{0, \xi}-\bar{U}_{0, \xi}\right|+\frac{1}{2} t\left|H_{0, \xi}-\bar{H}_{0, \xi}\right| .
$$

If $\tau \leq \bar{\tau} \leq t$ we have

$$
\begin{aligned}
\left|U_{\xi}(t)-\bar{U}_{\xi}(t)\right| \leq & \left|U_{\xi}(\bar{\tau})-\bar{U}_{\xi}(\bar{\tau})\right|+\frac{1}{2} \int_{\bar{\tau}}^{t}\left|V_{\xi}(s)-\bar{V}_{\xi}(s)\right| d s \\
\leq & \left|U_{\xi}(\bar{\tau})-\bar{U}_{\xi}(\bar{\tau})\right|+\frac{1}{2} \int_{\bar{\tau}}^{t}\left(|g(X(s))-g(\bar{X}(s))|+\left|y_{\xi}(s)-\bar{y}_{\xi}(s)\right|\right) d s \\
\leq & \left|U_{0, \xi}-\bar{U}_{0, \xi}\right|+\frac{1}{2} t\left|H_{0, \xi}-\bar{H}_{0, \xi}\right| \\
& +\frac{1}{2} \int_{0}^{t}\left(|g(X(s))-g(\bar{X}(s))|+\left|y_{\xi}(s)-\bar{y}_{\xi}(s)\right|\right) d s .
\end{aligned}
$$

Thus we have for any $\xi \in \mathbb{R}$ that,

$$
\left|U_{\xi}(t)-\bar{U}_{\xi}(t)\right| \leq\left|U_{0, \xi}-\bar{U}_{0, \xi}\right|+\frac{1}{2} t\left|H_{0, \xi}-\bar{H}_{0, \xi}\right|
$$




$$
+\frac{1}{2} \int_{0}^{t}\left(|g(X(s))-g(\bar{X}(s))|+\left|y_{\xi}(s)-\bar{y}_{\xi}(s)\right|\right) d s .
$$

Applying the $L^{2}(\mathbb{R})$-norm on both sides to the above inequality and (4.24), we obtain (4.23).

To simplify the remaining proofs we split for each time $t$ the real line into three parts: a continuous part where no wave breaking occured so far, a part where one solution already experienced wave breaking, and a part where both solutions have experienced wave breaking.

Definition 4.14. Define the disjoint sets

$$
\begin{aligned}
& R_{\text {cont }}(t)=\{\xi \in \mathbb{R} \mid t<\tau(\xi), \bar{\tau}(\xi)\}, \\
& R_{\text {mix }}(t)=\{\xi \in \mathbb{R} \mid \bar{\tau}(\xi) \leq t<\tau(\xi) \text { or } \tau(\xi) \leq t<\bar{\tau}(\xi)\}, \\
& R_{\text {disc }}(t)=\{\xi \in \mathbb{R} \mid \tau(\xi), \bar{\tau}(\xi) \leq t\},
\end{aligned}
$$

where the convention $\tau(\xi)=\infty$ for $\xi \in \Omega_{c}\left(X_{0}\right)$ is used.

Before the remaining terms in $\tilde{d}\left(S_{t}\left(X_{0}\right), S_{t}\left(\bar{X}_{0}\right) \bullet f\right)$ with $X_{0}, \bar{X}_{0} \in \mathcal{F}_{i, 0}^{\alpha, M}$ and $f \in$ $G$ will be handled, an estimate for the integral $\int_{0}^{t}\left\|V_{\xi}(s)-\bar{V}_{\xi}(s)\right\|_{1} d s$ is established. The following observations play an essential role therein. If $X_{0} \in \mathcal{F}_{i, 0}^{\alpha, M}$, then $U_{0, \xi}^{2} \leq y_{0, \xi} V_{0, \xi} \leq H_{0, \xi}$ since $0 \leq y_{0, \xi} \leq 1$. Hence

$$
\left\|U_{0, \xi}\right\|_{2} \leq \sqrt{\left\|H_{0, \xi}\right\|_{1}} \leq \sqrt{M},
$$

Moreover, from (2.14) we have that $U_{t}(\xi, t)=\frac{1}{2} V_{\xi}(\xi, t)-\frac{1}{4} V_{\infty}(t)$, and thus

$$
\left\|U_{t}\right\|_{\infty} \leq \frac{1}{4}\|V\|_{\infty} \leq \frac{1}{4} M
$$

since $V_{\xi}(t, \xi) \leq H_{0, \xi}(\xi)$ for all $\xi \in \mathbb{R}$.

Lemma 4.15. Given any two solutions $X(t)$ and $\bar{X}(t)$ with initial data $X_{0} \in \mathcal{F}_{i}^{\alpha, M}$ and $\bar{X}_{0} \in \mathcal{F}_{i, 0}^{\alpha, M}$, respectively, one has

$$
\begin{aligned}
\int_{0}^{t} \int_{\mathbb{R}} \mid V_{\xi}(\xi, s)- & \bar{V}_{\xi}(\xi, s) \mid d \xi d s \leq\left(8+3 t+2 t^{2}+\frac{3}{4} t^{3}\right)\left\|H_{0, \xi}-\bar{H}_{0, \xi}\right\|_{1} \\
+ & 2\left(\sqrt{1+\frac{1}{4} t^{2}}+\left(1+\frac{1}{4} t^{2}\right)\right) \sqrt{M}\left\|U_{0, \xi}-\bar{U}_{0, \xi}\right\|_{2} \\
+ & \left(4+t+t^{2}+\frac{1}{4} t^{3}\right) \sqrt{M}\left\|y_{0, \xi}-\bar{y}_{0, \xi}\right\|_{2} \\
+ & \left(\sqrt{1+\frac{1}{4} t^{2}}+1+\frac{1}{4} t^{2}\right) \sqrt{M} \\
& \times \int_{0}^{t}\|g(X(s))-g(\bar{X}(s))\|_{2}+\left\|y_{\xi}(s)-\bar{y}_{\xi}(s)\right\|_{2} d s
\end{aligned}
$$

Proof. We omit $\xi$ from the notation in this proof. Let $R_{\text {cont }}(t), R_{m i x}(t)$, and $R_{d i s c}(t)$ as in Definition 4.14. We want to estimate $\int_{0}^{t} \int_{\mathbb{R}}\left|V_{\xi}(\xi, s)-\bar{V}_{\xi}(\xi, s)\right| d \xi d s$ by the terms of $\tilde{d}\left(X_{0}, \bar{X}_{0}\right)$ and $\tilde{d}(X(t), \bar{X}(t))$. Since $R_{\text {cont }}(t), R_{\text {mix }}(t)$, and $R_{\text {disc }}(t)$ are disjoint, we can look at each region separately and add the results. 
If $\xi \in R_{\text {cont }}(t)$, then

$$
\int_{0}^{t}\left|V_{\xi}(\xi, s)-\bar{V}_{\xi}(\xi, s)\right| d s=t\left|H_{0, \xi}-\bar{H}_{0, \xi}\right| .
$$

Let $\xi \in R_{\text {mix }}(t)$, then there are two possibilities: either $\tau \leq t$, or $\bar{\tau} \leq t$. The two possibilities must be treated differently. To that end we split $R_{m i x}(t)$ into two disjoint sets,

$$
\begin{aligned}
& A(t)=\left\{\xi \in R_{\text {mix }}(t) \mid \tau(\xi) \leq t\right\}, \\
& \bar{A}(t)=\left\{\xi \in R_{\text {mix }}(t) \mid \bar{\tau}(\xi) \leq t\right\} .
\end{aligned}
$$

Assume first that $\xi \in \bar{A}(t)$, then

$$
\begin{aligned}
\int_{0}^{t}\left|V_{\xi}(s)-\bar{V}_{\xi}(s)\right| d s & =\int_{0}^{\bar{\tau}}\left|V_{\xi}(s)-\bar{V}_{\xi}(s)\right| d s+\int_{\bar{\tau}}^{t}\left|V_{\xi}(s)-\bar{V}_{\xi}(s)\right| d s \\
& =\bar{\tau}\left|H_{0, \xi}-\bar{H}_{0, \xi}\right|+\int_{\bar{\tau}}^{t}\left|H_{0, \xi}-\bar{V}_{\xi}(s)\right| d s .
\end{aligned}
$$

If $\bar{V}_{\xi}(s) \geq H_{0, \xi}$, then $\left|\bar{V}_{\xi}(s)-H_{0, \xi}\right| \leq\left|H_{0, \xi}-\bar{H}_{0, \xi}\right|$, and we can bound the integral by

$$
\int_{0}^{t}\left|V_{\xi}(s)-\bar{V}_{\xi}(s)\right| d s=t\left|H_{0, \xi}-\bar{H}_{0, \xi}\right|
$$

If $H_{0, \xi}>\bar{V}_{\xi}(s)$, then there are two cases to consider, namely $\xi \in \Omega_{c}\left(X_{0}\right)$ and $\xi \in \Omega_{d}\left(X_{0}\right)$. If $\xi \in \Omega_{c}\left(X_{0}\right)$ we have for $s \geq \bar{\tau}$,

$$
\left|H_{0, \xi}-\bar{V}_{\xi}(s)\right| \leq|g(X(s))-g(\bar{X}(s))|+\left|y_{\xi}(s)-\bar{y}_{\xi}(s)\right| .
$$

If, on the other hand, $\xi \in \Omega_{d}\left(X_{0}\right)$ we have for $s \geq \bar{\tau}$

$$
\begin{aligned}
H_{0, \xi}-\bar{V}_{\xi}(s) & =H_{0, \xi}-\bar{H}_{0, \xi}+\alpha(\bar{y}(\bar{\tau})) \bar{H}_{0, \xi} \\
& =(1-\alpha(\bar{y}(\bar{\tau})))\left(H_{0, \xi}-\bar{H}_{0, \xi}\right)+\alpha(\bar{y}(\bar{\tau})) H_{0, \xi}
\end{aligned}
$$

and hence by (2.4) and (2.9),

$$
\begin{aligned}
\int_{\bar{\tau}}^{t}\left|H_{0, \xi}-\bar{V}_{\xi}(s)\right| d s & \leq(t-\bar{\tau})\left|H_{0, \xi}-\bar{H}_{0, \xi}\right|+\alpha(\bar{y}(\bar{\tau})) \int_{\bar{\tau}}^{t} H_{0, \xi} d s \\
& \leq(t-\bar{\tau})\left|H_{0, \xi}-\bar{H}_{0, \xi}\right|+2 U_{\xi}(t)-2 U_{\xi}(\bar{\tau}) \\
& \leq(t-\bar{\tau})\left|H_{0, \xi}-\bar{H}_{0, \xi}\right|+2\left(\bar{U}_{\xi}(\bar{\tau})-U_{\xi}(\bar{\tau})\right) \\
& \leq(t-\bar{\tau})\left|H_{0, \xi}-\bar{H}_{0, \xi}\right|+2\left(\left|U_{0, \xi}-\bar{U}_{0, \xi}\right|+\frac{1}{2} \bar{\tau}\left|H_{0, \xi}-\bar{H}_{0, \xi}\right|\right) .
\end{aligned}
$$

Thus for $\xi \in \bar{A}(t)$ we have

$$
\begin{aligned}
\int_{0}^{t}\left|V_{\xi}(s)-\bar{V}_{\xi}(s)\right| d s \leq & 2 t\left|H_{0, \xi}-\bar{H}_{0, \xi}\right|+2\left|U_{0, \xi}-\bar{U}_{0, \xi}\right| \\
& +\int_{0}^{t}|g(X(s))-g(\bar{X}(s))|+\left|y_{\xi}(s)-\bar{y}_{\xi}(s)\right| d s .
\end{aligned}
$$

Since (2.32) implies that the measure of $\bar{A}(t)$ is bounded by $\left(1+\frac{1}{4} t^{2}\right) M$, we obtain $L^{2}(\mathbb{R})$-estimates on the right hand side of (4.41) when integrating over $\bar{A}(t)$. 
Assume now that $\xi \in A(t)$. Then

$$
\int_{0}^{t}\left|V_{\xi}(s)-\bar{V}_{\xi}(s)\right| d s=\tau\left|H_{0, \xi}-\bar{H}_{0, \xi}\right|+\int_{\tau}^{t}\left|V_{\xi}(s)-\bar{H}_{0, \xi}\right| d s .
$$

If $V_{\xi}(s) \geq \bar{H}_{0, \xi}$, then $\left|V_{\xi}(s)-\bar{H}_{0, \xi}\right| \leq\left|H_{0, \xi}-\bar{H}_{0, \xi}\right|$, and we can bound the integral by

$$
\int_{0}^{t}\left|V_{\xi}(s)-\bar{V}_{\xi}(s)\right| d s \leq t\left|H_{0, \xi}-\bar{H}_{0, \xi}\right| .
$$

Assume that $\bar{H}_{0, \xi}>V_{\xi}(s)$ and $s \geq \tau$. Since the measure of $A(t)$ is not bounded in terms of $M$ and $t$ only, see Corollary 2.4, we cannot use the same argument as for $\xi \in \bar{A}(t)$ to get $L^{2}(\mathbb{R})$-estimates. For $X_{0} \in \mathcal{F}_{i}^{\alpha, M}$ there exists $\hat{X}_{0} \in \mathcal{F}_{i, 0}^{\alpha, M}$ such that $\hat{X}_{0} \bullet f=X_{0}$, where $f=y_{0}+H_{0} \in G$. Then (2.30) gives

$$
\hat{H}_{0, \xi} \circ f=\frac{1}{1+\frac{1}{4} \hat{\tau}^{2} \circ f}=\frac{1}{1+\frac{1}{4} \tau^{2}},
$$

and in particular for $t \geq \tau$,

$$
1 \leq\left(1+\frac{1}{4} t^{2}\right) \hat{H}_{0, \xi} \circ f
$$

We want to estimate $\bar{H}_{0, \xi}-V_{\xi}(s)$. Assume that $\xi \in \Omega_{d}\left(\bar{X}_{0}\right)$, then

$$
\begin{aligned}
\bar{H}_{0, \xi}-V_{\xi}(s) & =\bar{H}_{0, \xi}-H_{0, \xi}+\alpha(y(\tau)) H_{0, \xi} \\
& =(1-\alpha(y(\tau)))\left(\bar{H}_{0, \xi}-H_{0, \xi}\right)+\alpha(y(\tau)) \bar{H}_{0, \xi},
\end{aligned}
$$

and hence by (2.4) and (2.9),

$$
\begin{aligned}
\int_{\tau}^{t}\left|V_{\xi}(s)-\bar{H}_{0, \xi}\right| d s & \leq(t-\tau)\left|H_{0, \xi}-\bar{H}_{0, \xi}\right|+\int_{\tau}^{t} \bar{H}_{0, \xi} d s \\
& =(t-\tau)\left|H_{0, \xi}-\bar{H}_{0, \xi}\right|+2\left(\bar{U}_{\xi}(t)-\bar{U}_{\xi}(\tau)\right) \\
& \leq(t-\tau)\left|H_{0, \xi}-\bar{H}_{0, \xi}\right|+2\left(U_{\xi}(\tau)-\bar{U}_{\xi}(\tau)\right) \\
& \leq(t-\tau)\left|H_{0, \xi}-\bar{H}_{0, \xi}\right|+2\left|U_{0, \xi}-\bar{U}_{0, \xi}\right|+\tau\left|H_{0, \xi}-\bar{H}_{0, \xi}\right| \\
& \leq t\left|H_{0, \xi}-\bar{H}_{0, \xi}\right|+2\left|U_{0, \xi}-\bar{U}_{0, \xi}\right| .
\end{aligned}
$$

Since $t \geq s \geq \tau$ we have that

$$
\begin{aligned}
\left|U_{0, \xi}-\bar{U}_{0, \xi}\right| \leq & \left(1+\frac{1}{4} t^{2}\right) \hat{H}_{0, \xi} \circ f\left|U_{0, \xi}-\bar{U}_{0, \xi}\right| \\
= & \left(1+\frac{1}{4} t^{2}\right)\left|\hat{U}_{0, \xi} \circ f f_{\xi} \hat{H}_{0, \xi} \circ f-\bar{U}_{0, \xi} \hat{H}_{0, \xi} \circ f\right| \\
= & \left(1+\frac{1}{4} t^{2}\right) \mid \hat{U}_{0, \xi} \circ f\left(\hat{H}_{0, \xi} \circ f f_{\xi}-\bar{H}_{0, \xi}\right) \\
& +\hat{U}_{0, \xi} \circ f \bar{H}_{0, \xi}-\bar{U}_{0, \xi} \hat{H}_{0, \xi} \circ f \mid \\
\leq & \left(1+\frac{1}{4} t^{2}\right)\left(\left|\hat{U}_{0, \xi} \circ f\right|\left|\hat{H}_{0, \xi} \circ f f_{\xi}-\bar{H}_{0, \xi}\right|\right. \\
& \left.\quad+\left|\hat{U}_{0, \xi} \circ f \bar{H}_{0, \xi}-\bar{U}_{0, \xi} \hat{H}_{0, \xi} \circ f\right|\right) .
\end{aligned}
$$


By definition $\hat{H}_{0, \xi} \circ f f_{\xi}=H_{0, \xi}$ and $\left|\hat{U}_{0, \xi} \circ f\right| \leq 1$, and thus

$$
\left|U_{0, \xi}-\bar{U}_{0, \xi}\right| \leq\left(1+\frac{1}{4} t^{2}\right)\left(\left|\hat{U}_{0, \xi} \circ f \bar{H}_{0, \xi}-\bar{U}_{0, \xi} \hat{H}_{0, \xi} \circ f\right|+\left|H_{0, \xi}-\bar{H}_{0, \xi}\right|\right) \text {. }
$$

To estimate the first term on the right hand side of (4.49) we note that $1=$ $1-f_{\xi}+f_{\xi}$, and hence

$$
\begin{aligned}
\left|\hat{U}_{0, \xi} \circ f \bar{H}_{0, \xi}-\bar{U}_{0, \xi} \hat{H}_{0, \xi} \circ f\right|= & \left|\hat{U}_{0, \xi} \circ f \bar{H}_{0, \xi}-\bar{U}_{0, \xi} \hat{H}_{0, \xi} \circ f\right|\left(\left(1-f_{\xi}\right)+f_{\xi}\right) \\
\leq & \left(\left|\hat{U}_{0, \xi} \circ f \bar{H}_{0, \xi}\right|+\left|\bar{U}_{0, \xi} \hat{H}_{0, \xi} \circ f\right|\right)\left(1-f_{\xi}\right) \\
& \quad+\left|\hat{U}_{0, \xi} \circ f f_{\xi} \bar{H}_{0, \xi}-\bar{U}_{0, \xi} \hat{H}_{0, \xi} \circ f f_{\xi}\right| \\
\leq & \left(\left|\bar{U}_{0, \xi}\right|+\bar{H}_{0, \xi}\right)\left(1-f_{\xi}\right)+\left|\bar{U}_{0, \xi}\right|\left|H_{0, \xi}-\bar{H}_{0, \xi}\right| \\
& +\bar{H}_{0, \xi}\left|U_{0, \xi}-\bar{U}_{0, \xi}\right| .
\end{aligned}
$$

Since $\bar{X}_{0} \in \mathcal{F}_{i, 0}^{\alpha, M}$, and $f=y_{0}+H_{0}$ we have that

$$
1-f_{\xi}=\bar{y}_{0, \xi}+\bar{H}_{0, \xi}-y_{0, \xi}-H_{0, \xi},
$$

and thus

$$
\begin{gathered}
\left|U_{0, \xi}-\bar{U}_{0, \xi}\right| \leq\left(1+\frac{1}{4} t^{2}\right)\left(4\left|H_{0, \xi}-\bar{H}_{0, \xi}\right|+\left(\left|\bar{U}_{0, \xi}\right|+\bar{H}_{0, \xi}\right)\left|y_{0, \xi}-\bar{y}_{0, \xi}\right|\right. \\
\left.+\bar{H}_{0, \xi}\left|U_{0, \xi}-\bar{U}_{0, \xi}\right|\right) .
\end{gathered}
$$

Inserting (4.52) into (4.47) and subsequently into (4.42), we end up with

$$
\begin{aligned}
\int_{0}^{t}\left|V_{\xi}(s)-\bar{H}_{0, \xi}\right| d s \leq & \left(8+2 t+2 t^{2}\right)\left|H_{0, \xi}-\bar{H}_{0, \xi}\right| \\
& +2\left(1+\frac{1}{4} t^{2}\right)\left(\left(\left|\bar{U}_{0, \xi}\right|+\bar{H}_{0, \xi}\right)\left|y_{0, \xi}-\bar{y}_{0, \xi}\right|\right. \\
& \left.+\bar{H}_{0, \xi}\left|U_{0, \xi}-\bar{U}_{0, \xi}\right|\right) .
\end{aligned}
$$

Assume that $\xi \in \Omega_{c}\left(\bar{X}_{0}\right)$. Then, by a similar argument as for (4.49),

$$
\begin{aligned}
\bar{H}_{0, \xi}-V_{\xi}(s) & \leq\left(\bar{H}_{0, \xi}-V_{\xi}(s)\right)\left(1+\frac{1}{4} t^{2}\right) \hat{H}_{0, \xi} \circ f \\
& =\left(1+\frac{1}{4} t^{2}\right)\left(\bar{H}_{0, \xi} \hat{H}_{0, \xi} \circ f-\hat{V}_{\xi} \circ f(s) \hat{H}_{0, \xi} \circ f f_{\xi}\right) \\
& =\left(1+\frac{1}{4} t^{2}\right)\left(\bar{H}_{0, \xi}\left(\hat{H}_{0, \xi} \circ f-\hat{V}_{\xi} \circ f(s)\right)+\hat{V}_{\xi} \circ f(s)\left(\bar{H}_{0, \xi}-H_{0, \xi}\right)\right) \\
(4.54) \quad & \leq\left(1+\frac{1}{4} t^{2}\right)\left(\left|H_{0, \xi}-\bar{H}_{0, \xi}\right|+\bar{H}_{0, \xi}\left(\hat{H}_{0, \xi} \circ f-\hat{V}_{\xi} \circ f(s)\right)\right) .
\end{aligned}
$$

Since $\bar{X}_{0} \in \mathcal{F}_{i, 0}^{\alpha, M}$, and $f=y_{0}+H_{0}$ we have that

$$
1-f_{\xi}=\bar{y}_{0, \xi}+\bar{H}_{0, \xi}-y_{0, \xi}-H_{0, \xi},
$$

and thus,

$$
\begin{aligned}
\left(\hat{H}_{0, \xi} \circ f-\hat{V}_{\xi} \circ f(s)\right) \bar{H}_{0, \xi}= & \left(\hat{H}_{0, \xi} \circ f-\hat{V}_{\xi} \circ f(s)\right) \bar{H}_{0, \xi}\left(1-f_{\xi}\right) \\
& +\left(H_{0, \xi}-V_{\xi}(s)\right) \bar{H}_{0, \xi}
\end{aligned}
$$




$$
\begin{aligned}
& \leq \bar{H}_{0, \xi}\left|1-f_{\xi}\right|+\left|H_{0, \xi}-\bar{H}_{0, \xi}\right|+\bar{H}_{0, \xi}\left(\bar{H}_{0, \xi}-V_{\xi}(s)\right) \\
& \leq\left(\bar{H}_{0, \xi}\left|y_{\xi}(s)-\bar{y}_{\xi}(s)\right|+\bar{H}_{0, \xi}|g(X(s))-g(\bar{X}(s))|\right) \\
& \quad+2\left|H_{0, \xi}-\bar{H}_{0, \xi}\right|+\bar{H}_{0, \xi}\left|y_{0, \xi}-\bar{y}_{0, \xi}\right| .
\end{aligned}
$$

Hence,

$$
\begin{aligned}
\int_{0}^{t}\left|V_{\xi}(s)-\bar{H}_{0, \xi}\right| d s & \leq 3 t\left(1+\frac{1}{4} t^{2}\right)\left|H_{0, \xi}-\bar{H}_{0, \xi}\right| \\
& +t\left(1+\frac{1}{4} t^{2}\right) \bar{H}_{0, \xi}\left|y_{0, \xi}-\bar{y}_{0, \xi}\right| \\
& +\left(1+\frac{1}{4} t^{2}\right) \bar{H}_{0, \xi} \int_{0}^{t}|g(X(s))-g(\bar{X}(s))|+\left|y_{\xi}(s)-\bar{y}_{\xi}(s)\right| d s .
\end{aligned}
$$

From (4.43), (4.53), and (4.57) we get that for $\xi \in A(t)$ the estimate

$$
\begin{aligned}
\int_{0}^{t}\left|V_{\xi}(s)-\bar{V}_{\xi}(s)\right| d s & \leq\left(8+3 t+2 t^{2}+\frac{3}{4} t^{3}\right)\left|H_{0, \xi}-\bar{H}_{0, \xi}\right| \\
& +2\left(1+\frac{1}{4} t^{2}\right) \bar{H}_{0, \xi}\left|U_{0, \xi}-\bar{U}_{0, \xi}\right| \\
& +\left(1+\frac{1}{4} t^{2}\right)\left(2\left|\bar{U}_{0, \xi}\right|+(2+t)\left|\bar{H}_{0, \xi}\right|\right)\left|y_{0, \xi}-\bar{y}_{0, \xi}\right| \\
& +\left(1+\frac{1}{4} t^{2}\right) \bar{H}_{0, \xi} \int_{0}^{t}|g(X(s))-g(\bar{X}(s))| d s \\
& +\left(1+\frac{1}{4} t^{2}\right) \bar{H}_{0, \xi} \int_{0}^{t}\left|y_{\xi}(s)-\bar{y}_{\xi}(s)\right| d s,
\end{aligned}
$$

holds.

Assume finally that $\xi \in R_{d i s c}(t)$. Recall that according to (2.32) the measure of $R_{\text {disc }}(t)$ is less than or equal to $\left(1+\frac{1}{4} t^{2}\right) M$. Since (4.40) and (4.47) give the same estimate we can assume without loss of generality that $\tau \leq \bar{\tau} \leq t$. By (4.47) we have

$$
\begin{aligned}
\int_{0}^{t}\left|V_{\xi}(s)-\bar{V}_{\xi}(s)\right| d s= & \int_{0}^{\tau}\left|H_{0, \xi}-\bar{H}_{0, \xi}\right| d s+\int_{\tau}^{\bar{\tau}}\left|V_{\xi}(s)-\bar{H}_{0, \xi}\right| d s \\
& +\int_{\bar{\tau}}^{t}\left|V_{\xi}(s)-\bar{V}_{\xi}(s)\right| d s \\
= & (\bar{\tau}+\tau)\left|H_{0, \xi}-\bar{H}_{0, \xi}\right|+2\left|U_{0, \xi}-\bar{U}_{0, \xi}\right| \\
& +\int_{\bar{\tau}}^{t}\left|V_{\xi}(s)-\bar{V}_{\xi}(s)\right| d s .
\end{aligned}
$$

For $s \geq \bar{\tau}$ we have

$$
\left|V_{\xi}(s)-\bar{V}_{\xi}(s)\right| \leq|g(X(s))-g(\bar{X}(s))|+\left|y_{\xi}(s)-\bar{y}_{\xi}(s)\right| .
$$

Thus

$$
\begin{aligned}
\int_{0}^{t}\left|V_{\xi}(s)-\bar{V}_{\xi}(s)\right| d s \leq & 2 t\left|H_{0, \xi}-\bar{H}_{0, \xi}\right|+2\left|U_{0, \xi}-\bar{U}_{0, \xi}\right| \\
& +\int_{0}^{t}|g(X(s))-g(\bar{X}(s))|+\left|y_{\xi}(s)-\bar{y}_{\xi}(s)\right| d s .
\end{aligned}
$$


If we collect the estimates (4.33), (4.41), (4.58), and (4.61) we get

$$
\begin{aligned}
\int_{0}^{t} \int_{\mathbb{R}}\left|V_{\xi}(\xi, s)-\bar{V}(\xi, s)\right| d \xi d s \leq & \left(8+3 t+2 t^{2}+\frac{3}{4} t^{3}\right)\left|H_{0, \xi}-\bar{H}_{0, \xi}\right| \\
& +2 \int_{\bar{A}(t) \cup R_{d i s c}(t)}\left|U_{0, \xi}-\bar{U}_{0, \xi}\right| d \xi \\
& +\int_{\bar{A}(t) \cup R_{d i s c}(t)} \int_{0}^{t}|g(X(s))-g(\bar{X}(s))| d s d \xi \\
& +\int_{\bar{A}(t) \cup R_{d i s c}(t)} \int_{0}^{t}\left|y_{\xi}(s)-\bar{y}_{\xi}(s)\right| d s d \xi \\
& +2\left(1+\frac{1}{4} t^{2}\right) \int_{A(t)}\left|U_{0, \xi}-\bar{U}_{0, \xi}\right| \bar{H}_{0, \xi} d \xi \\
& +2\left(1+\frac{1}{4} t^{2}\right) \\
& \times \int_{A(t)}\left(\left|\bar{U}_{0, \xi}\right|+\left(1+\frac{1}{2} t\right)\left|\bar{H}_{0, \xi}\right|\right)\left|y_{0, \xi}-\bar{y}_{0, \xi}\right| d \xi \\
& +\left(1+\frac{1}{4} t^{2}\right) \bar{H}_{0, \xi} \\
& \times \int_{A(t)} \int_{0}^{t}|g(X(s))-g(\bar{X}(s))| d s d \xi \\
+ & \left(1+\frac{1}{4} t^{2}\right) \bar{H}_{0, \xi} \int_{A(t)} \int_{0}^{t}\left|y_{\xi}(s)-\bar{y}_{\xi}(s)\right| d s d \xi
\end{aligned}
$$

From (2.32) we have that $\mathrm{m}\left(\bar{A}(t) \cup R_{\text {disc }}(t)\right) \leq\left(1+\frac{1}{4} t^{2}\right) M$, while (4.30) ensures that $\left\|\bar{U}_{0, \xi}\right\|_{2} \leq \sqrt{M}$. Thus

$$
\begin{aligned}
\int_{0}^{t} \int_{\mathbb{R}} \mid V_{\xi}(\xi, s)- & \bar{V}(\xi, s) \mid d \xi d s \leq\left(8+3 t+2 t^{2}+\frac{3}{4} t^{3}\right)\left\|H_{0, \xi}-\bar{H}_{0, \xi}\right\|_{1} \\
+ & 2\left(\sqrt{1+\frac{1}{4} t^{2}}+\left(1+\frac{1}{4} t^{2}\right)\right) \sqrt{M}\left\|U_{0, \xi}-\bar{U}_{0, \xi}\right\|_{2} \\
+ & \left(4+t+t^{2}+\frac{1}{4} t^{3}\right) \sqrt{M}\left\|y_{0, \xi}-\bar{y}_{0, \xi}\right\|_{2} \\
+ & \left(1+\frac{1}{4} t^{2}+\sqrt{1+\frac{1}{4} t^{2}}\right) \sqrt{M} \\
& \times \int_{0}^{t}\|g(X(s))-g(\bar{X}(s))\|_{2}+\left\|y_{\xi}(s)-\bar{y}_{\xi}(s)\right\|_{2} d s
\end{aligned}
$$

The system (2.14) and Lemma 4.15 can now be used to estimate the time evolution of $\|y(t)-\bar{y}(t)\|_{\infty},\|U(t)-\bar{U}(t)\|_{\infty}$, and $\left\|U(t) H_{0, \xi}-\bar{U}(t) \bar{H}_{0, \xi}\right\|_{2}$. Note that it is not necessary to derive an estimate for $\left\|H_{\xi}(t)-\bar{H}_{\xi}(t)\right\|_{1}$, since $H_{\xi}(\xi, t)=H_{0, \xi}(\xi)$ and $\bar{H}_{\xi}(\xi, t)=\bar{H}_{0, \xi}(\xi)$. 
Lemma 4.16. Let $X(t), \bar{X}(t)$ be solutions with initial data $X_{0} \in \mathcal{F}_{i}^{\alpha, M}$ and $\bar{X}_{0} \in$ $\mathcal{F}_{i, 0}^{\alpha, M}$, respectively. Then

$$
\begin{aligned}
\|U(t)-\bar{U}(t)\|_{\infty} & \leq\left\|U_{0}-\bar{U}_{0}\right\|_{\infty}+\frac{1}{4} \int_{0}^{t} \int_{\mathbb{R}}\left|V_{\xi}(\xi, s)-\bar{V}_{\xi}(\xi, s)\right| d \xi d s \\
\|y(t)-\bar{y}(t)\|_{\infty} & \leq\|y(0)-\bar{y}(0)\|_{\infty}+\int_{0}^{t}\|U(s)-\bar{U}(s)\|_{\infty} d s \\
\left\|U(t) H_{\xi}-\bar{U}(t) \bar{H}_{\xi}\right\|_{2} & \leq\left\|U_{0} H_{0, \xi}-\bar{U}_{0} \bar{H}_{0, \xi}\right\|_{2}+\frac{1}{4} M t\left\|H_{0, \xi}-\bar{H}_{0, \xi}\right\|_{2} \\
& +\frac{1}{4} \sqrt{M} \int_{0}^{t} \int_{\mathbb{R}}\left|V_{\xi}(\xi, s)-\bar{V}_{\xi}(\xi, s)\right| d \xi d s .
\end{aligned}
$$

Proof. We omit $\xi$ from the notation in this proof. It suffices to show the inequalities for $\|U(t)-\bar{U}(t)\|_{\infty}$ and $\left\|U(t) H_{0, \xi}-\bar{U}(t) \bar{H}_{0, \xi}\right\|_{2}$, since (4.65) follows immediately from (2.14). We have

$$
\begin{aligned}
\frac{d}{d t}|U(t)-\bar{U}(t)| & \leq\left|\frac{1}{2}(V(t)-\bar{V}(t))+\frac{1}{4}\left(V_{\infty}(t)-\bar{V}_{\infty}(t)\right)\right| \\
& \leq \frac{1}{4} \int_{-\infty}^{\infty}\left|V_{\xi}(t)-\bar{V}_{\xi}(t)\right| d \xi
\end{aligned}
$$

and from (4.31) there is

$$
\begin{aligned}
\frac{d}{d t}\left|U(t) H_{0, \xi}-\bar{U}(t) \bar{H}_{0, \xi}\right| & \leq\left|U_{t}(t)-\bar{U}_{t}(t)\right| \bar{H}_{0, \xi}+\left|U_{t}(t)\right|\left|H_{0, \xi}-\bar{H}_{0, \xi}\right| \\
& \leq \frac{1}{4} \int_{-\infty}^{\infty}\left|V_{\xi}(t)-\bar{V}_{\xi}(t)\right| d \xi \bar{H}_{0, \xi}+\frac{1}{4} M\left|H_{0, \xi}-\bar{H}_{0, \xi}\right|
\end{aligned}
$$

Since $\left\|\bar{H}_{0, \xi}\right\|_{2} \leq \sqrt{\left\|\bar{H}_{0, \xi}\right\|_{1}} \leq \sqrt{M}$ the lemma follows by applying the $L^{2}(\mathbb{R})$-norm to both sides of (4.67) and (4.68).

It remains to estimate the norms $\|g(X(t))-g(\bar{X}(t))\|_{2},\left\|g_{2}(X(t))-g_{2}(\bar{X}(t))\right\|_{2}$, and $\left\|g_{3}(X(t))-g_{3}(\bar{X}(t))\right\|_{2}$.

Lemma 4.17. Let $X(t), \bar{X}(t)$ be the solutions with initial data $X_{0} \in \mathcal{F}_{i}^{\alpha, M}$ and $\bar{X}_{0} \in \mathcal{F}_{i, 0}^{\alpha, M}$, respectively. Then

$$
\begin{aligned}
\left\|g_{2}(X(t))-g_{2}(\bar{X}(t))\right\|_{2} \leq & \left\|g_{2}\left(X_{0}\right)-g_{2}\left(\bar{X}_{0}\right)\right\|_{2}+\sqrt{M}\left\|\alpha^{\prime}\right\|_{\infty}\left\|H_{0, \xi}-\bar{H}_{0, \xi}\right\|_{1} \\
& +\left\|\alpha^{\prime}\right\|_{\infty} M\left(\left\|U_{0, \xi}-\bar{U}_{0, \xi}\right\|_{2}+\frac{1}{2} t\left\|H_{0, \xi}-\bar{H}_{0, \xi}\right\|_{2}\right), \\
\left\|g_{3}(X(t))-g_{3}(\bar{X}(t))\right\|_{2} \leq & \left\|g_{3}\left(X_{0}\right)-g_{3}\left(\bar{X}_{0}\right)\right\|_{2}+\frac{1}{4} t\left\|g_{2}\left(X_{0}\right)-g_{2}\left(\bar{X}_{0}\right)\right\|_{2} \\
& +\frac{1}{2} t\left\|\alpha^{\prime}\right\|_{\infty}\left\|U_{0} H_{0, \xi}-\bar{U}_{0} \bar{H}_{0, \xi}\right\|_{2} \\
& +\frac{1}{4}\left\|\alpha^{\prime}\right\|_{\infty} M t\left\|U_{0, \xi}-\bar{U}_{0, \xi}\right\|_{2} \\
& +\frac{1}{8}\left\|\alpha^{\prime}\right\|_{\infty} M t^{2}\left\|H_{0, \xi}-\bar{H}_{0, \xi}\right\|_{2} \\
& +\frac{1}{4} t\left\|\alpha^{\prime}\right\|_{\infty} \sqrt{M}\left\|H_{0, \xi}-\bar{H}_{0, \xi}\right\|_{1} \\
& +\frac{1}{4}\left\|\alpha^{\prime}\right\|_{\infty} \sqrt{M} \int_{0}^{t} \int_{\mathbb{R}}\left|V_{\xi}(\xi, s)-\bar{V}_{\xi}(\xi, s)\right| d \xi d s,
\end{aligned}
$$




$$
\begin{aligned}
\|g(X(t))-g(\bar{X}(t))\|_{2} \leq & \left\|g\left(X_{0}\right)-g\left(\bar{X}_{0}\right)\right\|_{2}+\left\|y_{0, \xi}-\bar{y}_{0, \xi}\right\|_{2} \\
& +\frac{1}{2} t\left\|\alpha^{\prime}\right\|_{\infty} M\left\|U_{0, \xi}-\bar{U}_{0, \xi}\right\|_{2} \\
& +\left(1+\frac{1}{4}\left\|\alpha^{\prime}\right\|_{\infty} M t^{2}\right)\left\|H_{0, \xi}-\bar{H}_{0, \xi}\right\|_{2} \\
& +\left\|\alpha^{\prime}\right\|_{\infty} \sqrt{M}\left\|y_{0}-\bar{y}_{0}\right\|_{\infty} \\
& +t\left\|\alpha^{\prime}\right\|_{\infty}\left\|U_{0} H_{0, \xi}-\bar{U}_{0} \bar{H}_{0, \xi}\right\|_{2} \\
& +2\left\|g_{3}\left(X_{0}\right)-g_{3}\left(\bar{X}_{0}\right)\right\|_{2} \\
& +\frac{1}{4} t\left\|g_{2}\left(X_{0}\right)-g_{2}\left(\bar{X}_{0}\right)\right\|_{2} \\
& +\frac{1}{4} t\left\|\alpha^{\prime}\right\|_{\infty} \sqrt{M}\left\|H_{0, \xi}-\bar{H}_{0, \xi}\right\|_{1} \\
& +\int_{0}^{t}\left\|U_{\xi}(s)-\bar{U}_{\xi}(s)\right\|_{2} d s \\
& +\left\|\alpha^{\prime}\right\|_{\infty} \sqrt{M} \int_{0}^{t}\|U(s)-\bar{U}(s)\|_{\infty} d s .
\end{aligned}
$$

Proof. We omit $\xi$ from the notation in this proof. Recall the splitting of $\mathbb{R}$ into $R_{\text {cont }}(t), R_{m i x}(t)$, and $R_{\text {disc }}(t)$ given in Definition 4.14,

Let us prove the estimate for $g_{2}$. Assume that $\xi \in R_{\text {cont }}(t)$, then if $\xi \in \Omega_{c}\left(X_{0}\right) \cap$ $\Omega_{c}\left(\bar{X}_{0}\right)$ we have

$$
g_{2}(X(t))-g_{2}(\bar{X}(t))=0-0=0 .
$$

We can assume without loss of generality that $\xi \in \Omega_{c}\left(X_{0}\right) \cap \Omega_{d}\left(\bar{X}_{0}\right)$ in the mixed case since the argument does not use the property $\bar{y}_{0}+\bar{H}_{0}=i d$. We have

$$
\left|g_{2}(X(t))-g_{2}(\bar{X}(t))\right|=\left\|\alpha^{\prime}\right\|_{\infty} \bar{H}_{\infty}\left|\bar{U}_{\xi}(t)\right|,
$$

and from (2.9) we know that $\left|\bar{U}_{\xi}(t)\right| \leq\left|\bar{U}_{0, \xi}\right|$, and hence

$$
\left|g_{2}(X(t))-g_{2}(\bar{X}(t))\right| \leq\left|g_{2}\left(X_{0}\right)-g_{2}\left(\bar{X}_{0}\right)\right| .
$$

If $\xi \in \Omega_{d}\left(X_{0}\right) \cap \Omega_{d}\left(\bar{X}_{0}\right)$, then (2.9) implies that

$$
\begin{aligned}
\left|g_{2}(X(t))-g_{2}(\bar{X}(t))\right| \leq & \left\|\alpha^{\prime}\right\|_{\infty}\left|H_{\infty}-\bar{H}_{\infty}\right|\left|\bar{U}_{\xi}(t)\right|+\left\|\alpha^{\prime}\right\|_{\infty} H_{\infty}\left|U_{\xi}(t)-\bar{U}_{\xi}(t)\right| \\
\leq & \left\|\alpha^{\prime}\right\|_{\infty}\left|\bar{U}_{0, \xi}\right|\left|H_{\infty}-\bar{H}_{\infty}\right| \\
& +\left\|\alpha^{\prime}\right\|_{\infty} M\left(\left|U_{0, \xi}-\bar{U}_{0, \xi}\right|+\frac{1}{2} t\left|H_{0, \xi}-\bar{H}_{0, \xi}\right|\right) .
\end{aligned}
$$

Assume that $\xi \in R_{m i x}(t)$. Then if $\xi \in \Omega_{c}\left(X_{0}\right) \cap \Omega_{d}\left(\bar{X}_{0}\right)$ or $\xi \in \Omega_{d}\left(X_{0}\right) \cap \Omega_{c}\left(\bar{X}_{0}\right)$ we have that

$$
g_{2}(X(t))-g_{2}(\bar{X}(t))=0-0=0 .
$$

If $\xi \in \Omega_{d}\left(X_{0}\right) \cap \Omega_{d}\left(\bar{X}_{0}\right)$, and if we assume without loss of generality that $\tau \leq t<\bar{\tau}$, then we have

$$
\left|g_{2}(X(t))-g_{2}(\bar{X}(t))\right|=\left\|\alpha^{\prime}\right\|_{\infty} \bar{H}_{\infty}\left|\bar{U}_{\xi}(t)\right|,
$$

and from (2.9) we know that $\left|\bar{U}_{\xi}(t)\right| \leq\left|\bar{U}_{\xi}(\tau)\right|$, and hence

$$
\left|g_{2}(X(t))-g_{2}(\bar{X}(t))\right| \leq\left\|\alpha^{\prime}\right\|_{\infty} \bar{H}_{\infty}\left|\bar{U}_{\xi}(\tau)-U_{\xi}(\tau)\right| .
$$


Thus following the same line as in (4.75), we end up with

$$
\left|g_{2}(X(t))-g_{2}(\bar{X}(t))\right| \leq\left\|\alpha^{\prime}\right\|_{\infty} M\left(\left|U_{0, \xi}-\bar{U}_{0, \xi}\right|+\frac{1}{2} t\left|H_{0, \xi}-\bar{H}_{0, \xi}\right|\right) .
$$

Assume that $\xi \in R_{\text {disc }}(t)$. Then

$$
g_{2}(X(t))-g_{2}(\bar{X}(t))=0-0=0 .
$$

From (4.72), (4.74), (4.75), (4.76), (4.79), and (4.80) we get

$$
\begin{aligned}
\left|g_{2}(X(t))-g_{2}(\bar{X}(t))\right| \leq & \left|g_{2}\left(X_{0}\right)-g_{2}\left(\bar{X}_{0}\right)\right| \\
& +\left\|\alpha^{\prime}\right\|_{\infty}\left|\bar{U}_{0, \xi}\right|\left|H_{\infty}-\bar{H}_{\infty}\right| \\
& +\left\|\alpha^{\prime}\right\|_{\infty} M\left|U_{0, \xi}-\bar{U}_{0, \xi}\right| \\
& +\frac{1}{2}\left\|\alpha^{\prime}\right\|_{\infty} M t\left|H_{0, \xi}-\bar{H}_{0, \xi}\right| .
\end{aligned}
$$

The estimate (4.69) is obtained after some direct computations by taking the $L^{2}(\mathbb{R})$ norm on both sides.

Next we show the inequality for $g_{3}$. Assume that $\xi \in R_{\text {cont }}(t)$, then if $\xi \in$ $\Omega_{c}\left(X_{0}\right) \cap \Omega_{c}\left(\bar{X}_{0}\right)$ we have

$$
g_{3}(X(t))-g_{3}(\bar{X}(t))=0-0=0 .
$$

For the mixed case assume without loss of generality $\xi \in \Omega_{c}\left(X_{0}\right) \cap \Omega_{d}\left(\bar{X}_{0}\right)$ since the argument does not depend on the property $\bar{y}_{0}+\bar{H}_{0}=i d$. We have

$$
\left|g_{3}(X(t))-g_{3}(\bar{X}(t))\right|=\left\|\alpha^{\prime}\right\|_{\infty}|\bar{U}(t)|\left|\bar{U}_{\xi}(t)\right|,
$$

and from (2.9) we know that $\left|\bar{U}_{\xi}(t)\right| \leq\left|\bar{U}_{0, \xi}\right|$, and hence by (4.31) we have

$$
\begin{aligned}
\left|g_{3}(X(t))-g_{3}(\bar{X}(t))\right| & =\left\|\alpha^{\prime}\right\|_{\infty}\left|\bar{U}(t) \| \bar{U}_{\xi}(t)\right| \\
& \leq\left\|\alpha^{\prime}\right\|_{\infty}|\bar{U}(t)|\left|\bar{U}_{0, \xi}\right| \\
& \leq\left\|\alpha^{\prime}\right\|_{\infty}\left|\bar{U}_{0}\right|\left|\bar{U}_{0, \xi}\right|+\frac{1}{4} t|| \alpha^{\prime} \| \bar{H}_{\infty}\left|\bar{U}_{0, \xi}\right| \\
& \leq\left|g_{3}\left(X_{0}\right)-g_{3}\left(\bar{X}_{0}\right)\right|+\frac{1}{4} t\left|g_{2}\left(X_{0}\right)-g_{2}\left(\bar{X}_{0}\right)\right| .
\end{aligned}
$$

If $\xi \in \Omega_{d}\left(X_{0}\right) \cap \Omega_{d}\left(\bar{X}_{0}\right)$, then

$$
\begin{aligned}
g_{3}(X(t))-g_{3}(\bar{X}(t))= & \left\|\alpha^{\prime}\right\|_{\infty} U(t) U_{\xi}(t)-\left\|\alpha^{\prime}\right\|_{\infty} \bar{U}(t) \bar{U}_{\xi}(t) \\
= & \left\|\alpha^{\prime}\right\|_{\infty}\left(U_{0}+\int_{0}^{t} U_{t}(s) d s\right)\left(U_{0, \xi}+\frac{1}{2} t H_{0, \xi}\right) \\
& -\left\|\alpha^{\prime}\right\|_{\infty}\left(\bar{U}_{0}+\int_{0}^{t} \bar{U}_{t}(s) d s\right)\left(\bar{U}_{0, \xi}+\frac{1}{2} t \bar{H}_{0, \xi}\right) \\
= & g_{3}\left(X_{0}\right)-g_{3}\left(\bar{X}_{0}\right)+\frac{1}{2} t\left\|\alpha^{\prime}\right\|_{\infty}\left(U_{0} H_{0, \xi}-\bar{U}_{0} \bar{H}_{0, \xi}\right) \\
& +\left(\bar{U}_{0, \xi}+\frac{1}{2} t \bar{H}_{0, \xi}\right)\left\|\alpha^{\prime}\right\|_{\infty} \int_{0}^{t}\left(U_{t}(s)-\bar{U}_{t}(s)\right) d s \\
& +\left\|\alpha^{\prime}\right\|_{\infty} \int_{0}^{t} U_{t}(s) d s\left(U_{0, \xi}+\frac{1}{2} t H_{0, \xi}-\bar{U}_{0, \xi}-\frac{1}{2} t \bar{H}_{0, \xi}\right) .
\end{aligned}
$$


Since (2.9) implies that $\left|\bar{U}_{\xi}(t)\right|=\left|\bar{U}_{0, \xi}+\frac{1}{2} t \bar{H}_{0, \xi}\right|$ is decreasing in time and due to (4.31) we have that

$$
\begin{aligned}
\left|g_{3}(X(t))-g_{3}(\bar{X}(t))\right| \leq & \left|g_{3}\left(X_{0}\right)-g_{3}\left(\bar{X}_{0}\right)\right|+\frac{1}{2} t\left\|\alpha^{\prime}\right\|_{\infty}\left|U_{0} H_{0, \xi}-\bar{U}_{0} \bar{H}_{0, \xi}\right| \\
& +\frac{1}{4}\left\|\alpha^{\prime}\right\|_{\infty} M t\left|U_{0, \xi}-\bar{U}_{0, \xi}\right|+\frac{1}{8}\left\|\alpha^{\prime}\right\|_{\infty} M t^{2}\left|H_{0, \xi}-\bar{H}_{0, \xi}\right| \\
& +\frac{1}{4}\left\|\alpha^{\prime}\right\|_{\infty}\left|\bar{U}_{0, \xi}+\frac{1}{2} t \bar{H}_{0, \xi}\right| \int_{0}^{t} \int_{\mathbb{R}}\left|V_{\xi}(\xi, s)-\bar{V}_{\xi}(\xi, s)\right| d \xi d s \\
\leq & \left|g_{3}\left(X_{0}\right)-g_{3}\left(\bar{X}_{0}\right)\right|+\frac{1}{2} t\left\|\alpha^{\prime}\right\|_{\infty}\left|U_{0} H_{0, \xi}-\bar{U}_{0} \bar{H}_{0, \xi}\right| \\
& +\frac{1}{4}\left\|\alpha^{\prime}\right\|_{\infty} M t\left|U_{0, \xi}-\bar{U}_{0, \xi}\right|+\frac{1}{8}\left\|\alpha^{\prime}\right\|_{\infty} M t^{2}\left|H_{0, \xi}-\bar{H}_{0, \xi}\right| \\
& +\frac{1}{4}\left\|\alpha^{\prime}\right\|_{\infty}\left|\bar{U}_{0, \xi}\right| \int_{0}^{t} \int_{\mathbb{R}}\left|V_{\xi}(\xi, s)-\bar{V}_{\xi}(\xi, s)\right| d \xi d s .
\end{aligned}
$$

Note that we can rewrite (4.85) in the following way,

$$
\begin{aligned}
g_{3}(X(t))-g_{3}(\bar{X}(t))= & g_{3}\left(X_{0}\right)-g_{3}\left(\bar{X}_{0}\right)+\frac{1}{2} t\left\|\alpha^{\prime}\right\|_{\infty}\left(U_{0} H_{0, \xi}-\bar{U}_{0} \bar{H}_{0, \xi}\right) \\
& +\left\|\alpha^{\prime}\right\|_{\infty}\left(U_{0, \xi}+\frac{1}{2} t H_{0, \xi}\right) \int_{0}^{t}\left(U_{t}(s)-\bar{U}_{t}(s)\right) d s \\
& +\left\|\alpha^{\prime}\right\|_{\infty} \int_{0}^{t} \bar{U}_{t}(s) d s\left(U_{0, \xi}+\frac{1}{2} t H_{0, \xi}-\bar{U}_{0, \xi}-\frac{1}{2} t \bar{H}_{0, \xi}\right),
\end{aligned}
$$

and hence

$$
\begin{aligned}
\left|g_{3}(X(t))-g_{3}(\bar{X}(t))\right| \leq & \left|g_{3}\left(X_{0}\right)-g_{3}\left(\bar{X}_{0}\right)\right|+\frac{1}{2} t\left\|\alpha^{\prime}\right\|_{\infty}\left|U_{0} H_{0, \xi}-\bar{U}_{0} \bar{H}_{0, \xi}\right| \\
& +\frac{1}{4}\left\|\alpha^{\prime}\right\|_{\infty} M t\left|U_{0, \xi}-\bar{U}_{0, \xi}\right|+\frac{1}{8}\left\|\alpha^{\prime}\right\|_{\infty} M t^{2}\left|H_{0, \xi}-\bar{H}_{0, \xi}\right| \\
& +\frac{1}{4}\left\|\alpha^{\prime}\right\|_{\infty}\left|U_{0, \xi}+\frac{1}{2} t H_{0, \xi}\right| \int_{0}^{t} \int_{\mathbb{R}}\left|V_{\xi}(\xi, s)-\bar{V}_{\xi}(\xi, s)\right| d \xi d s .
\end{aligned}
$$

Assume that $\xi \in \mathbb{R}_{m i x}(t)$. If $\xi \in \Omega_{c}\left(X_{0}\right) \cap \Omega_{d}\left(\bar{X}_{0}\right)$ or $\xi \in \Omega_{d}\left(X_{0}\right) \cap \Omega_{c}\left(\bar{X}_{0}\right)$, then

$$
g_{3}(X(t))-g_{3}(\bar{X}(t))=0-0=0 .
$$

Let $\xi \in \Omega_{d}\left(X_{0}\right) \cap \Omega_{d}\left(\bar{X}_{0}\right)$ and assume without loss of generality that $\tau \leq t<\bar{\tau}$. Then from (2.9) we know that $\left|\bar{U}_{\xi}(t)\right| \leq\left|\bar{U}_{\xi}(\tau)\right|$, and hence by (4.31)

$$
\begin{aligned}
\left|g_{3}(X(t))-g_{3}(\bar{X}(t))\right| & =\left\|\alpha^{\prime}\right\|_{\infty}|\bar{U}(t)|\left|\bar{U}_{\xi}(t)\right| \\
& \leq\left\|\alpha^{\prime}\right\|_{\infty}|\bar{U}(t)|\left|\bar{U}_{\xi}(\tau)\right| \\
& \leq\left\|\alpha^{\prime}\right\|_{\infty}|\bar{U}(\tau)|\left|\bar{U}_{\xi}(\tau)\right|+\frac{1}{4}(t-\tau)\left\|\alpha^{\prime}\right\| \bar{H}_{\infty}\left|\bar{U}_{\xi}(\tau)\right| \\
& \leq\left|g_{3}(X(\tau))-g_{3}(\bar{X}(\tau))\right|+\frac{1}{4}(t-\tau)\left|g_{2}(X(\tau))-g_{2}(\bar{X}(\tau))\right| .
\end{aligned}
$$

Combining (4.75) and (4.87) then yields

$$
\begin{aligned}
\left|g_{3}(X(t))-g_{3}(\bar{X}(t))\right| \leq & \left|g_{3}\left(X_{0}\right)-g_{3}\left(\bar{X}_{0}\right)\right|+\frac{1}{2} \tau\left\|\alpha^{\prime}\right\|_{\infty}\left|U_{0} H_{0, \xi}-\bar{U}_{0} \bar{H}_{0, \xi}\right| \\
& +\frac{1}{4}\left\|\alpha^{\prime}\right\|_{\infty} M \tau\left|U_{0, \xi}-\bar{U}_{0, \xi}\right|+\frac{1}{8}\left\|\alpha^{\prime}\right\|_{\infty} M \tau^{2}\left|H_{0, \xi}-\bar{H}_{0, \xi}\right|
\end{aligned}
$$




$$
\begin{aligned}
& +\frac{1}{4}\left\|\alpha^{\prime}\right\|_{\infty}\left|\bar{U}_{0, \xi}\right| \int_{0}^{\tau} \int_{\mathbb{R}}\left|V_{\xi}(\xi, s)-\bar{V}_{\xi}(\xi, s)\right| d \xi d s \\
& +\frac{1}{4}\left\|\alpha^{\prime}\right\|_{\infty}\left|\bar{U}_{0, \xi}\right|(t-\tau)\left|H_{\infty}-\bar{H}_{\infty}\right| \\
& +\frac{1}{4}\left\|\alpha^{\prime}\right\|_{\infty} M(t-\tau)\left(\left|U_{0, \xi}-\bar{U}_{0, \xi}\right|+\frac{1}{2} \tau\left|H_{0, \xi}-\bar{H}_{0, \xi}\right|\right),
\end{aligned}
$$

and thus

$$
\begin{aligned}
\left|g_{3}(X(t))-g_{3}(\bar{X}(t))\right| \leq & \left|g_{3}\left(X_{0}\right)-g_{3}\left(\bar{X}_{0}\right)\right|+\frac{1}{2} t\left\|\alpha^{\prime}\right\|_{\infty}\left|U_{0} H_{0, \xi}-\bar{U}_{0} \bar{H}_{0, \xi}\right| \\
& +\frac{1}{4}\left\|\alpha^{\prime}\right\|_{\infty} M t\left|U_{0, \xi}-\bar{U}_{0, \xi}\right|+\frac{1}{8}\left\|\alpha^{\prime}\right\|_{\infty} M t^{2}\left|H_{0, \xi}-\bar{H}_{0, \xi}\right| \\
& +\frac{1}{4}\left\|\alpha^{\prime}\right\|_{\infty}\left|\bar{U}_{0, \xi}\right| t\left|H_{\infty}-\bar{H}_{\infty}\right| \\
& +\frac{1}{4}\left\|\alpha^{\prime}\right\|_{\infty}\left|\bar{U}_{0, \xi}\right| \int_{0}^{t} \int_{\mathbb{R}}\left|V_{\xi}(\xi, s)-\bar{V}_{\xi}(\xi, s)\right| d \xi d s .
\end{aligned}
$$

Assume that $\xi \in R_{\text {disc }}(t)$. Then

$$
g_{3}(X(t))-g_{3}(\bar{X}(t))=0-0=0 .
$$

From (4.82), (4.84), (4.87), (4.90), (4.93), and (4.94) we get

$$
\begin{aligned}
\left|g_{3}(X(t))-g_{3}(\bar{X}(t))\right| \leq & \left|g_{3}\left(X_{0}\right)-g_{3}\left(\bar{X}_{0}\right)\right|+\frac{1}{4} t\left|g_{2}\left(X_{0}\right)-g_{2}\left(\bar{X}_{0}\right)\right| \\
& +\frac{1}{2} t\left\|\alpha^{\prime}\right\|_{\infty}\left|U_{0} H_{0, \xi}-\bar{U}_{0} \bar{H}_{0, \xi}\right| \\
& +\frac{1}{4}\left\|\alpha^{\prime}\right\|_{\infty} M t\left|U_{0, \xi}-\bar{U}_{0, \xi}\right| \\
& +\frac{1}{8}\left\|\alpha^{\prime}\right\|_{\infty} M t^{2}\left|H_{0, \xi}-\bar{H}_{0, \xi}\right| \\
& +\frac{1}{4}\left\|\alpha^{\prime}\right\|_{\infty}\left|\bar{U}_{0, \xi}\right| t\left|H_{\infty}-\bar{H}_{\infty}\right| \\
& +\frac{1}{4}\left\|\alpha^{\prime}\right\|_{\infty}\left|\bar{U}_{0, \xi}\right| \int_{0}^{t} \int_{\mathbb{R}}\left|V_{\xi}(\xi, s)-\bar{V}_{\xi}(\xi, s)\right| d \xi d s .
\end{aligned}
$$

The estimate (4.70) is obtained after some direct computations by taking the $L^{2}(\mathbb{R})$ norm on both sides.

We prove the estimate for $g$. Assume that $\xi \in R_{\text {cont }}(t)$, and let $\xi \in \Omega_{c}\left(X_{0}\right) \cap$ $\Omega_{c}\left(\bar{X}_{0}\right)$. Then

$$
\begin{aligned}
|g(X(t))-g(\bar{X}(t))| & =\left|H_{0, \xi}-\bar{H}_{0, \xi}+y_{\xi}(t)-\bar{y}_{\xi}(t)\right| \\
& \leq\left|g\left(X_{0}\right)-g\left(\bar{X}_{0}\right)\right|+\int_{0}^{t}\left|U_{\xi}(s)-\bar{U}_{\xi}(s)\right| d s .
\end{aligned}
$$

For the mixed case assume without loss of generality $\xi \in \Omega_{d}\left(X_{0}\right) \cap \Omega_{c}\left(\bar{X}_{0}\right)$, then (4.97)

$g(X(t))-g(\bar{X}(t))=g\left(X_{0}\right)-g\left(\bar{X}_{0}\right)+\int_{0}^{t}\left(U_{\xi}(s)-\bar{U}_{\xi}(s)\right) d s-\int_{0}^{t} \frac{d}{d s} \alpha(y(s)) H_{0, \xi} d s$.

We have

$$
\int_{0}^{t} \frac{d}{d s} \alpha(y(s)) H_{0, \xi} d s \leq \int_{0}^{t}\left\|\alpha^{\prime}\right\|_{\infty}|U(s)| H_{0, \xi} d s
$$




$$
\begin{aligned}
\leq & \int_{0}^{t}\left\|\alpha^{\prime}\right\|_{\infty}\left|U_{0}+\int_{0}^{s} U_{t}(\sigma) d \sigma\right| H_{0, \xi} d s \\
\leq & \left\|\alpha^{\prime}\right\|_{\infty}\left(\left|U_{0}\right| t+\frac{1}{8} H_{\infty} t^{2}\right) H_{0, \xi} \\
\leq & 2\left\|\alpha^{\prime}\right\|_{\infty}\left|U_{0}\right|\left(U_{\xi}(t)-U_{0, \xi}\right) \\
& +\frac{1}{4}\left\|\alpha^{\prime}\right\|_{\infty} t H_{\infty}\left(U_{\xi}(t)-U_{0, \xi}\right) \\
\leq & -2\left\|\alpha^{\prime}\right\|_{\infty}\left|U_{0}\right| U_{0, \xi}-\frac{1}{4} t\left\|\alpha^{\prime}\right\|_{\infty} H_{\infty} U_{0, \xi} \\
\leq & 2\left|g_{3}\left(X_{0}\right)-g_{3}\left(\bar{X}_{0}\right)\right|+\frac{1}{4} t\left|g_{2}\left(X_{0}\right)-g_{2}\left(\bar{X}_{0}\right)\right|,
\end{aligned}
$$

hence

$$
\begin{aligned}
|g(X(t))-g(\bar{X}(t))| \leq & \left|g\left(X_{0}\right)-g\left(\bar{X}_{0}\right)\right|+\int_{0}^{t}\left|U_{\xi}(s)-\bar{U}_{\xi}(s)\right| d s \\
& +2\left|g_{3}\left(X_{0}\right)-g_{3}\left(\bar{X}_{0}\right)\right|+\frac{1}{4} t\left|g_{2}\left(X_{0}\right)-g_{2}\left(\bar{X}_{0}\right)\right| .
\end{aligned}
$$

Here we used that $U_{0, \xi} \leq U_{\xi}(t) \leq 0$.

If $\xi \in \Omega_{d}\left(X_{0}\right) \cap \Omega_{d}\left(\bar{X}_{0}\right)$ we have

$$
\begin{aligned}
|g(X(t))-g(\bar{X}(t))|= & \left|y_{\xi}(t)-\bar{y}_{\xi}(t)+(1-\alpha(y(t))) H_{0, \xi}-(1-\alpha(\bar{y}(t))) \bar{H}_{0, \xi}\right| \\
\leq & \left|y_{0, \xi}-\bar{y}_{0, \xi}\right|+\int_{0}^{t}\left|U_{\xi}(s)-\bar{U}_{\xi}(s)\right| d s \\
& +\left|H_{0, \xi}-\bar{H}_{0, \xi}\right|+\left\|\alpha^{\prime}\right\|_{\infty} \bar{H}_{0, \xi}\left\|y_{0}-\bar{y}_{0}\right\|_{\infty} \\
& +\int_{0}^{t}\left\|\alpha^{\prime}\right\|_{\infty} \bar{H}_{0, \xi}\|U(s)-\bar{U}(s)\|_{\infty} d s .
\end{aligned}
$$

Assume that $\xi \in R_{\text {mix }}(t)$, and let $\xi \in \Omega_{c}\left(X_{0}\right) \cap \Omega_{d}\left(\bar{X}_{0}\right)$. Then

$$
\begin{aligned}
|g(X(t))-g(\bar{X}(t))|= & \left|y_{\xi}(t)-\bar{y}_{\xi}(t)+H_{0, \xi}-\bar{V}_{\xi}(t)\right| \\
\leq & |g(X(\bar{\tau}))-g(\bar{X}(\bar{\tau}))|+\int_{\bar{\tau}}^{t}\left|U_{\xi}(s)-\bar{U}_{\xi}(s)\right| d s \\
\leq & \left|g\left(X_{0}\right)-g\left(\bar{X}_{0}\right)\right|+2\left|g_{3}\left(X_{0}\right)-g_{3}\left(\bar{X}_{0}\right)\right| \\
& +\frac{1}{4} t\left|g_{2}\left(X_{0}\right)-g_{2}\left(\bar{X}_{0}\right)\right|+\int_{0}^{t}\left|U_{\xi}(s)-\bar{U}_{\xi}(s)\right| d s,
\end{aligned}
$$

where we used (4.99) in the last step. Both the argument and the result are the same in the case $\xi \in \Omega_{d}\left(X_{0}\right) \cap \Omega_{c}\left(\bar{X}_{0}\right)$.

If $\xi \in \Omega_{d}\left(X_{0}\right) \cap \Omega_{d}\left(\bar{X}_{0}\right)$, then if $\bar{\tau} \leq t<\tau$,

$$
\begin{aligned}
g(X(t))-g(\bar{X}(t))= & y_{\xi}(t)-\bar{y}_{\xi}(t)+H_{0, \xi}-\bar{H}_{0, \xi}+\alpha(\bar{y}(\bar{\tau})) \bar{H}_{0, \xi}-\alpha(y(t)) H_{0, \xi} \\
= & g(X(\bar{\tau}))-g(\bar{X}(\bar{\tau}))+\int_{\bar{\tau}}^{t}\left(U_{\xi}(s)-\bar{U}_{\xi}(s)\right) d s \\
& -\int_{\bar{\tau}}^{t} \frac{d}{d s} \alpha(y(s)) H_{0, \xi} d s .
\end{aligned}
$$

Then we can proceed as in (4.98), and apply (4.75), (4.86), and (4.100), to obtain

$$
|g(X(t))-g(\bar{X}(t))| \leq|g(X(\bar{\tau}))-g(\bar{X}(\bar{\tau}))|+2\left|g_{3}(X(\bar{\tau}))-g_{3}(\bar{X}(\bar{\tau}))\right|
$$




$$
\begin{aligned}
&+\frac{1}{4}(t-\bar{\tau})\left|g_{2}(X(\bar{\tau}))-g_{2}(\bar{X}(\bar{\tau}))\right|+\int_{\bar{\tau}}^{t}\left|U_{\xi}(s)-\bar{U}_{\xi}(s)\right| d s \\
& \leq\left|y_{0, \xi}-\bar{y}_{0, \xi}\right|+\int_{0}^{\bar{\tau}}\left|U_{\xi}(s)-\bar{U}_{\xi}(s)\right| d s+\left|H_{0, \xi}-\bar{H}_{0, \xi}\right| \\
&+\left\|\alpha^{\prime}\right\|_{\infty} \bar{H}_{0, \xi}\left\|y_{0}-\bar{y}_{0}\right\|_{\infty} \\
&+\left\|\alpha^{\prime}\right\|_{\infty} \bar{H}_{0, \xi} \int_{0}^{\bar{\tau}}\|U(s)-\bar{U}(s)\|_{\infty} d s \\
&+2\left|g_{3}\left(X_{0}\right)-g_{3}\left(\bar{X}_{0}\right)\right|+\bar{\tau}\left\|\alpha^{\prime}\right\|_{\infty}\left|U_{0} H_{0, \xi}-\bar{U}_{0} \bar{H}_{0, \xi}\right| \\
&+\frac{1}{2}\left\|\alpha^{\prime}\right\|_{\infty} M \bar{\tau}\left|U_{0, \xi}-\bar{U}_{0, \xi}\right|+\frac{1}{4}\left\|\alpha^{\prime}\right\|_{\infty} M \bar{\tau}^{2}\left|H_{0, \xi}-\bar{H}_{0, \xi}\right| \\
&+\frac{1}{2}\left\|\alpha^{\prime}\right\|_{\infty}\left|\bar{U}_{0, \xi}+\frac{1}{2} \bar{\tau} \bar{H}_{0, \xi}\right| \int_{0}^{\bar{\tau}} \int_{\mathbb{R}}\left|V_{\xi}(\xi, s)-\bar{V}_{\xi}(\xi, s)\right| d \xi d s \\
&+\frac{1}{4}\left\|\alpha^{\prime}\right\|_{\infty}\left|\bar{U}_{0, \xi}\right|(t-\bar{\tau})\left|H_{\infty}-\bar{H}_{\infty}\right| \\
&+\frac{1}{4}\left\|\alpha^{\prime}\right\|_{\infty} M(t-\bar{\tau})\left(\left|U_{0, \xi}-\bar{U}_{0, \xi}\right|+\frac{1}{2} \bar{\tau}\left|H_{0, \xi}-\bar{H}_{0, \xi}\right|\right) \\
&+\int_{\bar{\tau}}^{t}\left|U_{\xi}(s)-\bar{U}_{\xi}(s)\right| d s \\
& \leq\left|y_{0, \xi}-\bar{y}_{0, \xi}\right|+\frac{1}{2}\left\|\alpha^{\prime}\right\|_{\infty} M t\left|U_{0, \xi}-\bar{U}_{0, \xi}\right| \\
&+\left(1+\frac{1}{4}\left\|\alpha^{\prime}\right\|_{\infty} M t^{2}\right)\left|H_{0, \xi}-\bar{H}_{0, \xi}\right| \\
&+\int_{0}^{t}\left|U_{\xi}(s)-\bar{U}_{\xi}(s)\right| d s . \\
&+ 2\left|g_{3}\left(X_{0}\right)-g_{3}\left(\bar{X}_{0}\right)\right|+t\left\|\alpha^{\prime}\right\|_{\infty}\left|U_{0} H_{0, \xi}-\bar{U}_{0} \bar{H}_{0, \xi}\right| \\
&++\alpha^{\prime}\left\|_{\infty} \bar{H}_{0, \xi}\right\| y_{0}-\bar{y}_{0} \|_{\infty} \\
&+\left\|\alpha^{\prime}\right\|_{\infty}\left|\bar{U}_{0, \xi}\right| t\left|H_{\infty}-\bar{H}_{\infty}\right| \\
& \bar{H}_{0, \xi} \int_{0}^{t}\left\|U(s)-\bar{U}_{(s)}\right\|_{\infty} d s \\
&
\end{aligned}
$$

The term

$$
\frac{1}{2}\left\|\alpha^{\prime}\right\|_{\infty}\left|\bar{U}_{0, \xi}+\frac{1}{2} \bar{\tau} \bar{H}_{0, \xi}\right| \int_{0}^{t} \int_{\mathbb{R}}\left|V_{\xi}(\xi, s)-\bar{V}_{\xi}(\xi, s)\right| d \xi d s,
$$

vanishes since $\bar{U}_{0, \xi}+\frac{1}{2} \bar{\tau} \bar{H}_{0, \xi}=0$ by the definition of $\bar{\tau}$.

The case $\tau \leq t<\bar{\tau}$ is similar. Combining (4.89) and $U_{0, \xi}+\frac{1}{2} \tau H_{0, \xi}=0$, we get that

$$
\begin{aligned}
\left|g_{3}(X(\tau))-g_{3}(\bar{X}(\tau))\right| \leq & \left|g_{3}\left(X_{0}\right)-g_{3}\left(\bar{X}_{0}\right)\right|+\frac{1}{2} \tau\left\|\alpha^{\prime}\right\|_{\infty}\left|U_{0} H_{0, \xi}-\bar{U}_{0} \bar{H}_{0, \xi}\right| \\
& +\frac{1}{4}\left\|\alpha^{\prime}\right\|_{\infty} M \tau\left(\left|U_{0, \xi}-\bar{U}_{0, \xi}\right|+\frac{1}{2} \tau\left|H_{0, \xi}-\bar{H}_{0, \xi}\right|\right) .
\end{aligned}
$$

Thus, following the same lines as in (4.103) yields,

$$
|g(X(t))-g(\bar{X}(t))| \leq\left|y_{0, \xi}-\bar{y}_{0, \xi}\right|+\frac{1}{2}\left\|\alpha^{\prime}\right\|_{\infty} M t\left|U_{0, \xi}-\bar{U}_{0, \xi}\right|
$$




$$
\begin{aligned}
& +\left(1+\frac{1}{4}\left\|\alpha^{\prime}\right\|_{\infty} M t^{2}\right)\left|H_{0, \xi}-\bar{H}_{0, \xi}\right| \\
& +2\left|g_{3}\left(X_{0}\right)-g_{3}\left(\bar{X}_{0}\right)\right|+t\left\|\alpha^{\prime}\right\|_{\infty}\left|U_{0} H_{0, \xi}-\bar{U}_{0} \bar{H}_{0, \xi}\right| \\
& +\left\|\alpha^{\prime}\right\|_{\infty} \bar{H}_{0, \xi}\left\|y_{0}-\bar{y}_{0}\right\|_{\infty} \\
& +\frac{1}{4}\left\|\alpha^{\prime}\right\|_{\infty}\left|\bar{U}_{0, \xi}\right| t\left|H_{\infty}-\bar{H}_{\infty}\right| \\
& +\left\|\alpha^{\prime}\right\|_{\infty} \bar{H}_{0, \xi} \int_{0}^{t}\|U(s)-\bar{U}(s)\|_{\infty} d s \\
& +\int_{0}^{t}\left|U_{\xi}(s)-\bar{U}_{\xi}(s)\right| d s .
\end{aligned}
$$

Assume that $\xi \in R_{d i s c}(t)$, and assume without loss of generality that $\bar{\tau} \leq \tau$, then

$$
|g(X(t))-g(\bar{X}(t))| \leq|g(X(\tau))-g(\bar{X}(\tau))|+\int_{\tau}^{t}\left|U_{\xi}(s)-\bar{U}_{\xi}(s)\right| d s .
$$

Thus, from (4.103) and (4.106), we get that

$$
\begin{aligned}
|g(X(t))-g(\bar{X}(t))| \leq & \left|y_{0, \xi}-\bar{y}_{0, \xi}\right|+\frac{1}{2}\left\|\alpha^{\prime}\right\|_{\infty} M t\left|U_{0, \xi}-\bar{U}_{0, \xi}\right| \\
& +\left(1+\frac{1}{4}\left\|\alpha^{\prime}\right\|_{\infty} M t^{2}\right)\left|H_{0, \xi}-\bar{H}_{0, \xi}\right| \\
& +2\left|g_{3}\left(X_{0}\right)-g_{3}\left(\bar{X}_{0}\right)\right|+t\left\|\alpha^{\prime}\right\|_{\infty}\left|U_{0} H_{0, \xi}-\bar{U}_{0} \bar{H}_{0, \xi}\right| \\
& +\left\|\alpha^{\prime}\right\|_{\infty} \bar{H}_{0, \xi}\left\|y_{0}-\bar{y}_{0}\right\|_{\infty} \\
& +\frac{1}{4}\left\|\alpha^{\prime}\right\|_{\infty}\left|\bar{U}_{0, \xi}\right| t\left|H_{\infty}-\bar{H}_{\infty}\right| \\
& +\left\|\alpha^{\prime}\right\|_{\infty} \bar{H}_{0, \xi} \int_{0}^{t}\|U(s)-\bar{U}(s)\|_{\infty} d s \\
& +\int_{0}^{t}\left|U_{\xi}(s)-\bar{U}_{\xi}(s)\right| d s .
\end{aligned}
$$

Combining (4.96), (4.99), (4.100), (4.101), 4.103), (4.106), and (4.108) yields

$$
\begin{aligned}
|g(X(t))-g(\bar{X}(t))| \leq & \left|y_{0, \xi}-\bar{y}_{0, \xi}\right| \\
& +\frac{1}{2}\left\|\alpha^{\prime}\right\|_{\infty} M t\left|U_{0, \xi}-\bar{U}_{0, \xi}\right| \\
& +\left(1+\frac{1}{4}\left\|\alpha^{\prime}\right\|_{\infty} M t^{2}\right)\left|H_{0, \xi}-\bar{H}_{0, \xi}\right| \\
& +\left\|\alpha^{\prime}\right\|_{\infty} \bar{H}_{0, \xi}\left\|y_{0}-\bar{y}_{0}\right\|_{\infty} \\
& +t\left\|\alpha^{\prime}\right\|_{\infty}\left|U_{0} H_{0, \xi}-\bar{U}_{0} \bar{H}_{0, \xi}\right| \\
& +\left|g\left(X_{0}\right)-g\left(\bar{X}_{0}\right)\right|+2\left|g_{3}\left(X_{0}\right)-g_{3}\left(\bar{X}_{0}\right)\right| \\
& +\frac{1}{4} t\left|g_{2}\left(X_{0}\right)-g_{2}\left(\bar{X}_{0}\right)\right| \\
& +\int_{0}^{t}\left|U_{\xi}(s)-\bar{U}_{\xi}(s)\right| d s \\
& +\frac{1}{4}\left\|\alpha^{\prime}\right\|_{\infty}\left|\bar{U}_{0, \xi}\right| t\left\|H_{0, \xi}-\bar{H}_{0, \xi}\right\|_{1}
\end{aligned}
$$




$$
+\left\|\alpha^{\prime}\right\|_{\infty} \bar{H}_{0, \xi} \int_{0}^{t}\|U(s)-\bar{U}(s)\|_{\infty} d s
$$

The result is obtained after some direct computations by applying the $L^{2}(\mathbb{R})$-norm to both sides of the above estimate and using (4.30).

A Lipschitz theorem can now be stated for the flow in Lagrangian coordinates. The idea is to apply the estimates in Lemmas 4.13, 4.15, 4.16, and 4.17 together with Gronwall's inequality.

Theorem 4.18. For solutions $X(t), \bar{X}(t)$ of (2.14) with initial data $X_{0} \in \mathcal{F}_{i}^{\alpha, M}$ and $\bar{X}_{0} \in \mathcal{F}_{i, 0}^{\alpha, M}$ the estimate

$$
\tilde{d}(X(t), \bar{X}(t)) \leq C_{M, \alpha}(t) \tilde{d}\left(X_{0}, \bar{X}_{0}\right)
$$

holds, with $C_{M, \alpha}(t)=\tilde{C}_{M, \alpha}(t) e^{t \bar{C}_{M, \alpha}(t)}$, where

$$
\begin{aligned}
& \tilde{C}_{M, \alpha}(t)=3+\frac{3}{2} t+\frac{1}{2} t^{2}+\frac{3}{16} t^{3}+\sqrt{M}\left(1+\frac{1}{4} t+\frac{1}{4} t^{2}+\frac{1}{16} t^{3}\right) \\
& (4.111)+\left\|\alpha^{\prime}\right\|_{\infty} \sqrt{M}\left(5+2 t+t^{2}+\frac{3}{8} t^{3}\right)+\left\|\alpha^{\prime}\right\|_{\infty} M\left(3+\frac{5}{4} t+\frac{1}{2} t^{2}+\frac{1}{8} t^{3}\right), \\
& (4.112) \\
& \bar{C}_{M, \alpha}(t)=2+\left\|\alpha^{\prime}\right\|_{\infty} \sqrt{M}+\sqrt{M}\left(\frac{1}{2}+\frac{1}{8} t+\frac{1}{16} t^{2}\right)+\left\|\alpha^{\prime}\right\|_{\infty} M\left(1+\frac{1}{4} t+\frac{1}{8} t^{2}\right) .
\end{aligned}
$$

Proof. Combining Lemma 4.13, 4.16, and 4.17 we end up with

$$
\begin{aligned}
\tilde{d}(X(t), \bar{X}(t)) \leq & \tilde{d}\left(X_{0}, \bar{X}_{0}\right) \\
& +\left\|y_{0, \xi}-\bar{y}_{0, \xi}\right\|_{2} \\
& +\left\|\alpha^{\prime}\right\|_{\infty} M\left(1+\frac{3}{4} t\right)\left\|U_{0, \xi}-\bar{U}_{0, \xi}\right\|_{2} \\
& +\left(1+\frac{1}{2} t+\left\|\alpha^{\prime}\right\|_{\infty} M\left(\frac{3}{4} t+\frac{3}{8} t^{2}\right)\right)\left\|H_{0, \xi}-\bar{H}_{0, \xi}\right\|_{2} \\
& +\left\|\alpha^{\prime}\right\|_{\infty} \sqrt{M}\left\|y_{0}-\bar{y}_{0}\right\|_{\infty} \\
& +\left\|\alpha^{\prime}\right\|_{\infty} \sqrt{M}\left(1+\frac{1}{2} t\right)\left\|H_{0, \xi}-\bar{H}_{0, \xi}\right\|_{1} \\
& +\frac{3}{2} t\left\|\alpha^{\prime}\right\|_{\infty}\left\|U_{0} H_{0, \xi}-\bar{U}_{0} \bar{H}_{0, \xi}\right\|_{2} \\
& +\frac{1}{2} t\left\|g_{2}\left(X_{0}\right)-g_{2}\left(\bar{X}_{0}\right)\right\|_{2} \\
& +2\left\|g_{3}\left(X_{0}\right)-g_{3}\left(\bar{X}_{0}\right)\right\|_{2} \\
& +2 \int_{0}^{t}\left\|U_{\xi}(s)-\bar{U}_{\xi}(s)\right\|_{2} d s \\
& +\left(1+\left\|\alpha^{\prime}\right\|_{\infty} \sqrt{M}\right) \int_{0}^{t}\|U(s)-\bar{U}(s)\|_{\infty} d s \\
& +\frac{1}{2} \int_{0}^{t}\left(\|g(X(s))-g(\bar{X}(s))\|_{2}+\left\|y_{\xi}(s)-\bar{y}_{\xi}(s)\right\|_{2}\right) d s \\
& +\left(\frac{1}{4}+\frac{1}{2}\left\|\alpha^{\prime}\right\|_{\infty} \sqrt{M}\right) \int_{0}^{t} \int_{\mathbb{R}}\left|V_{\xi}(\xi, s)-\bar{V}_{\xi}(\xi, s)\right| d \xi d s
\end{aligned}
$$


Inserting the estimate from Lemma 4.15 yields

$$
\tilde{d}(X(t), \bar{X}(t)) \leq \tilde{C}_{M, \alpha}(t) \tilde{d}\left(X_{0}, \bar{X}_{0}\right)+\bar{C}_{M, \alpha}(t) \int_{0}^{t} \tilde{d}(X(s), \bar{X}(s)) d s
$$

with

$$
\begin{aligned}
& \tilde{C}_{M, \alpha}(t)=3+\frac{3}{2} t+\frac{1}{2} t^{2}+\frac{3}{16} t^{3}+\sqrt{M}\left(1+\frac{1}{4} t+\frac{1}{4} t^{2}+\frac{1}{16} t^{3}\right) \\
& (4.115)+\left\|\alpha^{\prime}\right\|_{\infty} \sqrt{M}\left(5+2 t+t^{2}+\frac{3}{8} t^{3}\right)+\left\|\alpha^{\prime}\right\|_{\infty} M\left(3+\frac{5}{4} t+\frac{1}{2} t^{2}+\frac{1}{8} t^{3}\right), \\
& (4.116) \\
& \bar{C}_{M, \alpha}(t)=2+\left\|\alpha^{\prime}\right\|_{\infty} \sqrt{M}+\sqrt{M}\left(\frac{1}{2}+\frac{1}{8} t+\frac{1}{16} t^{2}\right)+\left\|\alpha^{\prime}\right\|_{\infty} M\left(1+\frac{1}{4} t+\frac{1}{8} t^{2}\right) .
\end{aligned}
$$

The theorem follows from Gronwall's inequality with $C_{M, \alpha}(t)=\tilde{C}_{M, \alpha}(t) e^{t \bar{C}_{M, \alpha}(t)}$.

We are now ready to prove the Lipschitz continuity on $\mathcal{F}_{i, 0}^{\alpha, M}$. The ingredients for the proof are Theorem 4.18 and Lemma 4.12.

Theorem 4.19. Let $X, \bar{X} \in \mathcal{F}_{i, 0}^{\alpha, M}$, then for all $t \geq 0$ it holds that

$$
d_{M}(t, X, \bar{X}) \leq \hat{C}_{M, \alpha}(t) d_{M}(0, X, \bar{X}),
$$

where $\hat{C}_{M, \alpha}(t)$ is given by $\hat{C}_{M, \alpha}=e^{\frac{1}{2} t} \tilde{C}_{M, \alpha}(t) e^{t \bar{C}_{M, \alpha}(t)}$,

$$
\tilde{C}_{M, \alpha}(t)=3+\frac{3}{2} t+\frac{1}{2} t^{2}+\frac{3}{16} t^{3}+\sqrt{M}\left(1+\frac{1}{4} t+\frac{1}{4} t^{2}+\frac{1}{16} t^{3}\right)
$$

$$
\bar{C}_{M, \alpha}(t)=2+\left\|\alpha^{\prime}\right\|_{\infty} \sqrt{M}+\sqrt{M}\left(\frac{1}{2}+\frac{1}{8} t+\frac{1}{16} t^{2}\right)+\left\|\alpha^{\prime}\right\|_{\infty} M\left(1+\frac{1}{4} t+\frac{1}{8} t^{2}\right) .
$$

Proof. Let $1>\varepsilon>0$ and $X, \bar{X} \in \mathcal{F}_{i, 0}^{\alpha, M}$ be given and choose $\left\{X_{n}\right\}_{n=0}^{N}$ in $\mathcal{F}_{i, 0}^{\alpha, M}$, $\left\{f_{n}\right\}_{n=1}^{N}$, and $\left\{g_{n}\right\}_{n=0}^{N-1}$ in $G$ such that $X_{0}=X, X_{N}=\bar{X}$ and $d_{M}(0, X, \bar{X})+\varepsilon \geq$ $\sum_{n=1}^{N} \tilde{d}\left(X_{n} \bullet f_{n}, X_{n-1}\right)+\tilde{d}\left(X_{n}, X_{n-1} \bullet g_{n-1}\right)$. Then from the definition of $d_{M}$ we have

$$
\begin{aligned}
d_{M}(t, X, \bar{X}) & \leq \sum_{n=1}^{N} J\left(\Pi S_{t}\left(X_{n}\right), \Pi S_{t}\left(X_{n-1}\right)\right) \\
& \leq e^{\frac{1}{2} t} \sum_{n=1}^{N} J\left(S_{t}\left(X_{n}\right), S_{t}\left(X_{n-1}\right)\right),
\end{aligned}
$$

by Lemma 4.12, From the definition of $J$ and Theorem 4.18 we get

$$
\begin{aligned}
d_{M}(t, X, \bar{X}) & \leq e^{\frac{1}{2} t} \sum_{n=1}^{N} J\left(S_{t}\left(X_{n}\right), S_{t}\left(X_{n-1}\right)\right) \\
& \leq e^{\frac{1}{2} t} C_{M, \alpha}(t) \sum_{n=1}^{N}\left(\tilde{d}\left(X_{n} \bullet f_{n}, X_{n-1}\right)+\tilde{d}\left(X_{n}, X_{n-1} \bullet g_{n-1}\right)\right)
\end{aligned}
$$




$$
\leq \hat{C}_{M, \alpha}(t)\left(d_{M}(0, X, \bar{X})+\varepsilon\right) .
$$

The inequality holds for each $\varepsilon$ in the range $(0,1)$, which implies that

$$
d_{M}\left(\Pi S_{t}(X(t)), \Pi S_{t}(\bar{X}(t))\right) \leq \hat{C}_{M, \alpha}(t) d_{M}(0, X, \bar{X}) .
$$

Since $\mathcal{F}_{i, 0}^{\alpha, M}$ is in one to one correspondance with $\mathcal{D}_{0}^{\alpha, M}$ the metric $d_{M}$ on $\mathcal{F}_{i, 0}^{\alpha, M}$ induces a metric $d_{\mathcal{D}_{M}}$ on $\mathcal{D}_{0}^{\alpha, M}$.

Definition 4.20. Let $(u, \rho, \nu, \mu),(\bar{u}, \bar{\rho}, \bar{\nu}, \bar{\mu}) \in \mathcal{D}_{0}^{\alpha, M}$, then define $d_{\mathcal{D}_{0}^{\alpha, M}}:[0, \infty) \times$ $\mathcal{D}_{0}^{\alpha, M} \times \mathcal{D}_{0}^{\alpha, M} \rightarrow \mathbb{R}$ by

$$
d_{\mathcal{D}_{0}^{\alpha, M}}(t,(u, \rho, \nu, \mu),(\bar{u}, \bar{\rho}, \bar{\nu}, \bar{\mu}))=d_{M}(t, L((u, \rho, \nu, \mu)), L((\bar{u}, \bar{\rho}, \bar{\nu}, \bar{\mu})))
$$

for any $(u, \rho, \nu, \mu),(\bar{u}, \bar{\rho}, \bar{\nu}, \bar{\mu}) \in \mathcal{D}_{0}^{\alpha, M}$ and $t \geq 0$.

Theorem 4.21. The $\alpha$-dissipative solution operator $T_{t}$ is Lipschitz continuous in the sense that for any $\left(u_{0}, \rho_{0}, \nu_{0}, \mu_{0}\right),\left(\bar{u}_{0}, \bar{\rho}_{0}, \bar{\nu}_{0}, \bar{\mu}_{0}\right) \in \mathcal{D}_{0}^{\alpha, M}$ the inequality

$$
\begin{aligned}
& d_{\mathcal{D}_{0}^{\alpha, M}}\left(t,\left(u_{0}, \rho_{0}, \nu_{0}, \mu_{0}\right),\left(\bar{u}_{0}, \bar{\rho}_{0}, \bar{\nu}_{0}, \bar{\mu}_{0}\right)\right) \\
& \quad \leq \hat{C}_{M, \alpha}(t) d_{\mathcal{D}_{0}^{\alpha, M}}\left(0,\left(u_{0}, \rho_{0}, \nu_{0}, \mu_{0}\right),\left(\bar{u}_{0}, \bar{\rho}_{0}, \bar{\nu}_{0}, \bar{\mu}_{0}\right)\right)
\end{aligned}
$$

holds. The Lipschitz constant $\hat{C}_{M, \alpha}(t)$ is given inTheorem 4.19 .

Proof. The theorem follows from Theorem 3.14 and Theorem 4.19

\section{Appendix A. Examples}

Example A.1. In this example we construct an $\alpha$-dissipative solution. Let the initial data $\left(u_{0}, \rho_{0}, \nu_{0}, \mu_{0}\right) \in \mathcal{D}_{0}^{\alpha}$ be given by

$$
\begin{aligned}
u_{0}(x) & = \begin{cases}0, & x \leq-1, \\
x+1, & -1 \leq x \leq 0, \\
-x+1, & 0 \leq x \leq 1, \\
0, & 1 \leq x,\end{cases} \\
\rho_{0}(x) & =0, \\
\nu_{0} & =\mu_{0, a c}=u_{0, x}^{2}(x) d x=\mu_{0},
\end{aligned}
$$

and $\alpha(x)=\frac{x^{2}}{x^{2}+1}$. Then the initial data in Lagrangian coordinates is given by $\left(y_{0}, U_{0}, H_{0}, r_{0}, V_{0}\right)=L\left(\left(u_{0}, \rho_{0}, \nu_{0}, \mu_{0}\right)\right)$ where

$$
\begin{aligned}
& y_{0}(\xi)= \begin{cases}\xi, & \xi \leq-1, \\
\frac{1}{2}(\xi-1), & -1 \leq \xi \leq 3, \\
\xi-2, & 3 \leq \xi,\end{cases} \\
& H_{0}(\xi)= \begin{cases}0, & \xi \leq-1, \\
\frac{1}{2}(\xi+1), & -1 \leq \xi \leq 3, \\
2, & 3 \leq \xi,\end{cases}
\end{aligned}
$$




$$
\begin{aligned}
& U_{0}(\xi)= \begin{cases}0, & \xi \leq-1, \\
\frac{1}{2}(\xi+1), & -1 \leq \xi \leq 1, \\
\frac{1}{2}(-\xi+3), & 1 \leq \xi \leq 3, \\
0, & 3 \leq \xi,\end{cases} \\
& r_{0}(\xi)=0, \\
& V_{0}(\xi)=H_{0}(\xi) \text {. }
\end{aligned}
$$

Moreover the wave breaking time $\tau$ as a function of $\xi$ is given by

$$
\tau(\xi)= \begin{cases}\infty, & \xi<1 \\ 2, & 1<\xi<3 \\ \infty, & 3<\xi\end{cases}
$$

Then for $t<2$ the solution is given by

$$
\begin{aligned}
y(\xi, t) & = \begin{cases}\xi-\frac{1}{4} t^{2}, & \xi \leq-1, \\
\frac{1}{2}(\xi-1)+\frac{1}{2}(\xi+1) t+\frac{1}{8}(\xi-1) t^{2}, & -1 \leq \xi \leq 1, \\
\frac{1}{2}(\xi-1)-\frac{1}{2}(\xi-3) t+\frac{1}{8}(\xi-1) t^{2}, & 1 \leq \xi \leq 3, \\
\xi-2+\frac{1}{4} t^{2}, & 3 \leq \xi,\end{cases} \\
U(\xi, t) & = \begin{cases}-\frac{1}{2} t, & \xi \leq-1, \\
\frac{1}{2}(\xi+1)+\frac{1}{4}(\xi-1) t, & -1 \leq \xi \leq 1, \\
-\frac{1}{2}(\xi-1)+\frac{1}{4}(\xi-1) t, & 1 \leq \xi \leq 3, \\
\frac{1}{2} t, & 3 \leq \xi,\end{cases} \\
H(\xi, t) & =H_{0}(\xi), \\
r(\xi, t) & =0 \\
V(\xi, t) & =H_{0}(\xi) .
\end{aligned}
$$

In Eulerian coordinates the solution for $t<2$ is given by

$$
\begin{aligned}
& u(x, t)= \begin{cases}-\frac{1}{2} t, & x \leq-\frac{1}{4} t^{2}-1, \\
\frac{x-\frac{1}{2} t+1}{1+\frac{1}{2} t}, & -\frac{1}{4} t^{2}-1 \leq x \leq t, \\
\frac{-x+\frac{1}{2} t+1}{1-\frac{1}{2} t}, & t \leq x \leq \frac{1}{4} t^{2}+1, \\
\frac{1}{2} t, & \frac{1}{4} t^{2}+1 \leq x,\end{cases} \\
& d \nu(t)=d \mu(t)=u_{x}^{2}(t) d x
\end{aligned}
$$

As $t \rightarrow 2$ we note that $y(\xi, t) \rightarrow 2$ for $\xi \in(1,3)$. Hence all wave breaking takes place at the coordinates $(x=2, t=2)$. We have

$$
\alpha(2)=\frac{4}{5}
$$

At $t=2$ the solution in Lagrangian coordinates reads

$$
y(\xi, 2)= \begin{cases}\xi-1, & \xi \leq-1 \\ 2 \xi, & -1 \leq \xi \leq 1, \\ 2, & 1 \leq \xi \leq 3 \\ \xi-1, & 3 \leq \xi\end{cases}
$$




$$
\begin{aligned}
U(\xi, 2) & = \begin{cases}-1, & \xi \leq-1, \\
\xi, & -1 \leq \xi \leq 1, \\
1, & 1 \leq \xi \leq 3, \\
1, & 3 \leq \xi,\end{cases} \\
H(\xi, 2) & =H_{0}(\xi), \\
r(\xi, 2) & =0, \\
V(\xi, 2) & = \begin{cases}0, & \xi \leq-1, \\
\frac{1}{2}(\xi+1), & -1 \leq \xi \leq 1, \\
\frac{1}{10}(\xi+9), & 1 \leq \xi \leq 3, \\
\frac{6}{5}, & 3 \leq \xi,\end{cases}
\end{aligned}
$$

and thus the solution in Lagrangian coordinates for $t \geq 2$ is given by

$$
\begin{aligned}
y(\xi, t) & = \begin{cases}-\frac{3}{20} t^{2}-\frac{2}{5} t+\xi+\frac{2}{5}, & \xi \leq-1, \\
\frac{1}{2}\left(\xi-\frac{1}{5}\right)+\frac{1}{2}\left(\xi+\frac{1}{5}\right) t+\frac{1}{8}\left(\xi-\frac{1}{5}\right) t^{2}, & -1 \leq \xi \leq 1, \\
\frac{1}{10}(\xi+3)-\frac{1}{10}(\xi-7) t+\frac{1}{40}(\xi+3) t^{2}, & 1 \leq \xi \leq 3, \\
\frac{3}{20} t^{2}+\frac{2}{5} t+\xi-\frac{12}{5}, & 3 \leq \xi,\end{cases} \\
U(\xi, t) & = \begin{cases}-\frac{3}{10} t-\frac{2}{5}, & \xi \leq-1, \\
\frac{1}{2}\left(\xi+\frac{1}{5}\right)+\frac{1}{4}\left(\xi-\frac{1}{5}\right) t, & -1 \leq \xi \leq 1, \\
-\frac{1}{10}(\xi-7)+\frac{1}{20}(\xi+3) t, & 1 \leq \xi \leq 3, \\
\frac{3}{10} t+\frac{2}{5}, & 3 \leq \xi,\end{cases} \\
H(\xi, t)=H(\xi, 0), & \\
r(\xi, t)= & 0,
\end{aligned}
$$

$$
V(\xi, t)= \begin{cases}0, & \xi \leq-1, \\ \frac{1}{2}(\xi+1), & -1 \leq \xi \leq 1, \\ \frac{1}{10}(\xi+9), & 1 \leq \xi \leq 3, \\ \frac{6}{5}, & 3 \leq \xi .\end{cases}
$$

The solution in Eulerian coordinates for $t \geq 2$ is then given by

$$
\begin{aligned}
u(x, t)= & \begin{cases}-\frac{3}{10} t-\frac{2}{5}, & x \leq-\frac{3}{20} t^{2}-\frac{2}{5} t-\frac{3}{5}, \\
\frac{x+\frac{1}{5}\left(1-\frac{1}{2} t\right)}{1+\frac{1}{2} t}, & -\frac{3}{20} t^{2}-\frac{2}{5} t-\frac{3}{5} \leq x \leq \frac{1}{10} t^{2}+\frac{3}{5} t+\frac{2}{5}, \\
\frac{x-\frac{1}{2} t-1}{\frac{1}{2} t-1}, & \frac{1}{10} t^{2}+\frac{3}{5} t+\frac{2}{5} \leq x \leq \frac{3}{20} t^{2}+\frac{2}{5} t+\frac{3}{5}, \\
\frac{3}{10} t+\frac{2}{5}, & \frac{3}{20} t^{2}+\frac{2}{5} t+\frac{3}{5} \leq x,\end{cases} \\
\rho(x, t)= & 0, \\
d \nu(t)= & u_{x}(x, t)^{2} d x \\
& +\delta_{(x=2, t=2)}+\frac{4}{\left(\frac{1}{2} t-1\right)^{2}} \mathbf{1}_{\left[\frac{1}{10} t^{2}+\frac{3}{5} t+\frac{2}{5}, \frac{3}{20} t^{2}+\frac{2}{5} t+\frac{3}{5}\right]} d x \\
d \mu(t)= & u_{x}(x, t)^{2} d x+\frac{1}{5} \delta_{(x=2, t=2)} .
\end{aligned}
$$

In Figure 0 curves of $y(\xi, t)$ are drawn for $\xi=-1,1,3$. The cumulative energy function for the measures $\mu$ and $\nu$ are plotted for $t=0,2,4$ in Figure 3 . 


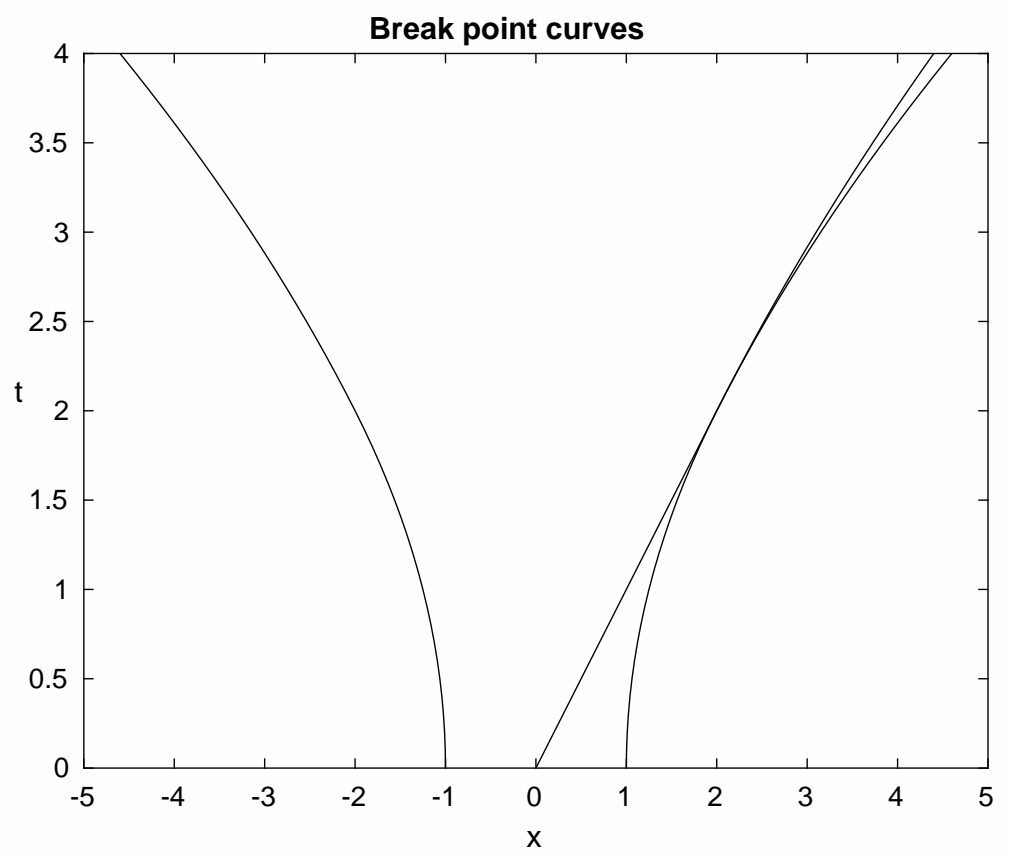

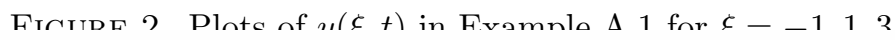
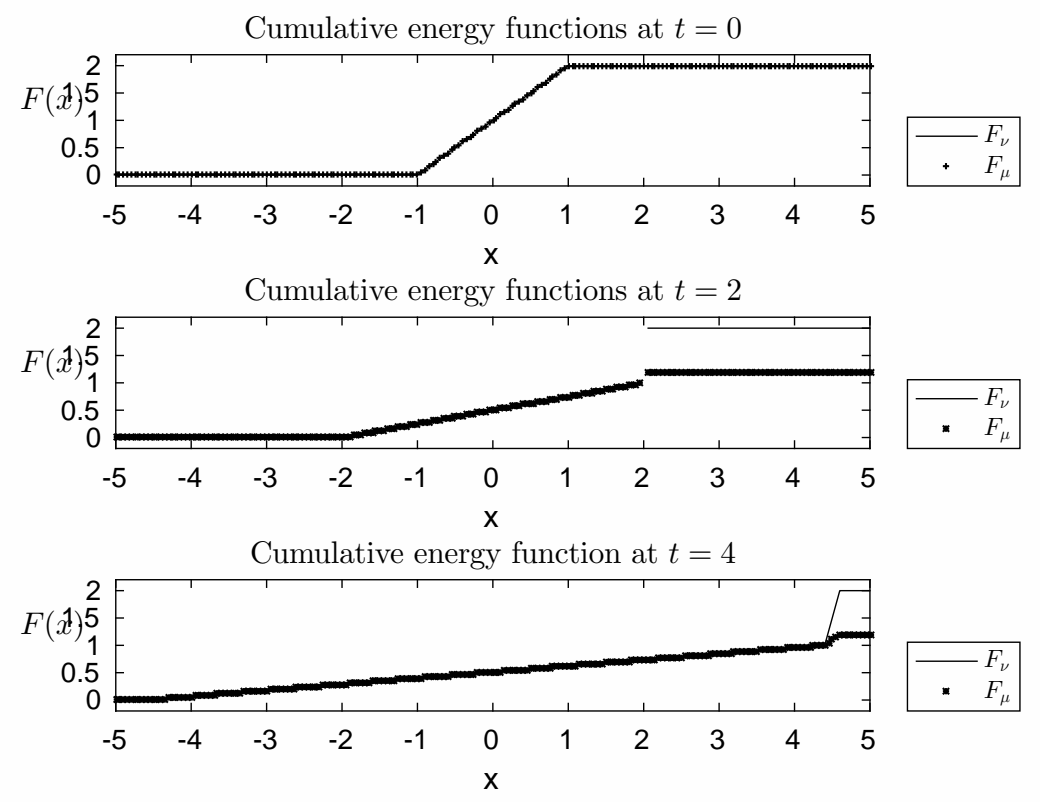

Figure 3. Plots of the cumulative energy functions in Example A.1 for $t=0,2,4$. 
Example A.2. In this example we show that the function $g$ in the construction of the metric is necessary. Let $\alpha \in[0,1]$ be a given constant, choose $\varepsilon \in(0,1)$, and let $\left(u_{0}, \rho_{0}, \nu_{0}, \mu_{0}\right)$ and $\left(\bar{u}_{0}, \bar{\rho}_{0}, \bar{\nu}_{0}, \bar{\mu}_{0}\right)$ be given by

$$
\begin{aligned}
& u_{0}(x)= \begin{cases}0, & x \leq-1, \\
x+1, & -1 \leq x \leq 0, \\
1-x, & 0 \leq x \leq \varepsilon, \\
1-\varepsilon, & \varepsilon \leq x\end{cases} \\
& \bar{u}_{0}(x)= \begin{cases}-2 \varepsilon, & x \leq-1, \\
x+1-2 \varepsilon, & -1 \leq x \leq \varepsilon, \\
1-\varepsilon, & \varepsilon \leq x,\end{cases} \\
& \rho_{0}=\bar{\rho}_{0}=0 \text {, } \\
& \nu_{0}=\bar{\nu}_{0}=u_{0, x}^{2} d x=\bar{\mu}_{0}=\mu_{0} .
\end{aligned}
$$

Then $X_{0}=L\left(\left(u_{0}, \rho_{0}, \nu_{0}, \mu_{0}\right)\right)$ and $\bar{X}_{0}=L\left(\left(\bar{u}_{0}, \bar{\rho}_{0}, \bar{\nu}_{0}, \bar{\mu}_{0}\right)\right)$ are given by

$$
\begin{gathered}
y_{0}(\xi)=\bar{y}_{0}(\xi)= \begin{cases}\xi, & \xi \leq-1, \\
\frac{1}{2}(\xi-1), & -1 \leq \xi \leq 1+2 \varepsilon, \\
\xi-1-\varepsilon, & 1+2 \varepsilon \leq \xi\end{cases} \\
U_{0}(\xi)= \begin{cases}0, & \xi \leq-1, \\
\frac{1}{2}(\xi+1), & -1 \leq \xi \leq 1, \\
-\frac{1}{2}(\xi-3), & 1 \leq \xi \leq 1+2 \varepsilon, \\
1-\varepsilon, & 1+2 \varepsilon \leq \xi,\end{cases} \\
\bar{U}_{0}(\xi)= \begin{cases}-2 \varepsilon, & \xi \leq-1, \\
\frac{1}{2}(\xi+1-4 \varepsilon), & -1 \leq \xi \leq 1+2 \varepsilon, \\
1-\varepsilon, & 1+2 \varepsilon \leq \xi,\end{cases} \\
H_{0}(\xi)=\bar{H}_{0}(\xi)= \begin{cases}0, & \xi \leq-1, \\
\frac{1}{2}(\xi+1), & -1 \leq \xi \leq 1+2 \varepsilon, \\
1+\varepsilon, & 1+2 \varepsilon \leq \xi,\end{cases} \\
r_{0}(\xi)=\bar{r}_{0}(\xi)=0 .
\end{gathered}
$$

Let $X(t)=S_{t}\left(X_{0}\right)$ and $\bar{X}(t)=S_{t}\left(\bar{X}_{0}\right)$, then for $0 \leq t<2$ we have

$$
\begin{aligned}
& y(\xi, t)= \begin{cases}\xi-\frac{1}{8}(1+\varepsilon) t^{2}, & \xi \leq-1, \\
\frac{1}{2}(\xi-1)+\frac{1}{2}(\xi+1) t+\frac{1}{8}(\xi-\varepsilon) t^{2}, & -1 \leq \xi \leq 1, \\
\frac{1}{2}(\xi-1)-\frac{1}{2}(\xi-3) t+\frac{1}{8}(\xi-\varepsilon) t^{2}, & 1 \leq \xi \leq 1+2 \varepsilon, \\
\xi-1-\varepsilon+(1-\varepsilon) t+\frac{1}{8}(1+\varepsilon) t^{2}, & 1+2 \varepsilon \leq \xi\end{cases} \\
& \bar{y}(\xi, t)= \begin{cases}\xi-2 \varepsilon t-\frac{1}{8}(1+\varepsilon) t^{2}, & \xi \leq-1, \\
\frac{1}{2}(\xi-1)+\frac{1}{2}(\xi+1-4 \varepsilon) t+\frac{1}{8}(\xi-\varepsilon) t^{2}, & -1 \leq \xi \leq 1+2 \varepsilon, \\
\xi-1-\varepsilon+(1-\varepsilon) t+\frac{1}{8}(1+\varepsilon) t^{2}, & 1+2 \varepsilon \leq \xi,\end{cases}
\end{aligned}
$$




$$
\begin{aligned}
& U(\xi, t)= \begin{cases}-\frac{1}{4}(1+\varepsilon) t, & \xi \leq-1, \\
\frac{1}{2}(\xi+1)+\frac{1}{4}(\xi-\varepsilon) t, & -1 \leq \xi \leq 1, \\
-\frac{1}{2}(\xi-3)+\frac{1}{4}(\xi-\varepsilon) t, & 1 \leq \xi \leq 1+2 \varepsilon, \\
1-\varepsilon+\frac{1}{4}(1+\varepsilon) t, & 1+2 \varepsilon \leq \xi\end{cases} \\
& \bar{U}(\xi, t)= \begin{cases}-2 \varepsilon-\frac{1}{4}(1+\varepsilon) t, & \xi \leq-1, \\
\frac{1}{2}(\xi+1)-2 \varepsilon+\frac{1}{4}(\xi-\varepsilon) t, & -1 \leq \xi \leq 1+2 \varepsilon, \\
1-\varepsilon+\frac{1}{4}(1+\varepsilon) t, & 1+2 \varepsilon \leq \xi\end{cases}
\end{aligned}
$$

Then $M(X(t))$, and $M(\bar{X}(t))$, the solutions in Eulerian coordinates, are given by

$$
\begin{aligned}
& x_{1}(t)=-1-\frac{1}{8}(1+\varepsilon) t^{2}, \\
& x_{2}(t)=t+\frac{1}{8}(1-\varepsilon) t^{2}, \\
& x_{3}(t)=\varepsilon+(1-\varepsilon) t+\frac{1}{8}(1+\varepsilon) t^{2}, \\
& u(x, t)= \begin{cases}-\frac{1}{4}(1+\varepsilon) t, & x \leq x_{1}(t), \\
\frac{1}{1+\frac{1}{2} t}\left(x+1-\frac{1}{4}(1+\varepsilon) t\right), & x_{1}(t) \leq x \leq x_{2}(t), \\
\frac{1}{1-\frac{1}{2} t}\left(1-x+\frac{1}{4}(3-\varepsilon) t\right), & x_{2}(t) \leq x \leq x_{3}(t), \\
1-\varepsilon+\frac{1}{4}(1+\varepsilon) t, & x_{3}(t) \leq x,\end{cases} \\
& \rho(x, t)=0 \text {, } \\
& d \nu(t)=\frac{1}{1+t+\frac{1}{4} t^{2}} \mathbf{1}_{\left[x_{1}(t), x_{2}(t)\right]} d x+\frac{1}{1-t+\frac{1}{4} t^{2}} \mathbf{1}_{\left[x_{2}(t), x_{3}(t)\right]} d x, \\
& \mu(t)=\nu(t), \\
& \bar{x}_{1}(t)=-1-2 \varepsilon t-\frac{1}{8}(1+\varepsilon) t^{2}, \\
& \bar{x}_{2}(t)=\varepsilon+(1-\varepsilon) t+\frac{1}{8}(1+\varepsilon) t^{2}, \\
& \bar{u}(x, t)= \begin{cases}-2 \varepsilon-\frac{1}{4}(1+\varepsilon) t, & x \leq \bar{x}_{1}(t), \\
\frac{1}{1+\frac{1}{2} t}\left(x+1-2 \varepsilon-\frac{1}{4} t(1-3 \varepsilon)\right), & \bar{x}_{1}(t) \leq x \leq \bar{x}_{2}(t), \\
1-\varepsilon+\frac{1}{4}(1+\varepsilon) t, & \bar{x}_{2}(t) \leq x,\end{cases} \\
& \bar{\rho}(x, t)=0, \\
& d \bar{\nu}(t)=\frac{1}{1+t+\frac{1}{4} t^{2}} \mathbf{1}_{\left[\bar{x}_{1}(t), \bar{x}_{2}(t)\right]} d x, \\
& \bar{\mu}(t)=\bar{\nu}(t),
\end{aligned}
$$

For $2 \leq t$ the solutions in Lagrangian coordinates are given by

$$
y(\xi, t)= \begin{cases}\xi+\frac{\alpha \varepsilon}{2}-\frac{\alpha \varepsilon}{2} t-\frac{1}{8}(1+(1-\alpha) \varepsilon) t^{2}, & \xi \leq-1, \\ \frac{1}{2}(\xi-1)+\frac{\alpha \varepsilon}{2}+\frac{1}{2}(\xi+1-\alpha \varepsilon) t+\frac{1}{8}(\xi-(1-\alpha) \varepsilon) t^{2}, & -1 \leq \xi \leq 1, \\ \frac{1-\alpha}{2}(\xi-1)+\frac{\alpha \varepsilon}{2}+\left(-\frac{1-\alpha}{2}(\xi-1)+1-\frac{\alpha \varepsilon}{2}\right) t & \\ \quad+\frac{1}{8}((1-\alpha)(\xi-1-\varepsilon)+1) t^{2}, & 1 \leq \xi \leq 1+2 \varepsilon, \\ \xi-1-\varepsilon-\frac{\alpha \varepsilon}{2}+\left(1-\varepsilon+\frac{\alpha \varepsilon}{2}\right) t+\frac{1}{8}(1+(1-\alpha) \varepsilon) t^{2}, & 1+2 \varepsilon \leq \xi,\end{cases}
$$


(A.60)

$$
\bar{y}(\xi, t)= \begin{cases}\xi-2 \varepsilon t-\frac{1}{8}(1+\varepsilon) t^{2}, & \xi \leq-1, \\ \frac{1}{2}(\xi-1)+\frac{1}{2}(\xi+1-4 \varepsilon) t+\frac{1}{8}(\xi-\varepsilon) t^{2}, & -1 \leq \xi \leq 1+2 \varepsilon, \\ \xi-1-\varepsilon+(1-\varepsilon) t+\frac{1}{8}(1+\varepsilon) t^{2}, & 1+2 \varepsilon \leq \xi,\end{cases}
$$

$$
U(\xi, t)= \begin{cases}-\frac{\alpha \varepsilon}{2}-\frac{1}{4}(1+(1-\alpha) \varepsilon) t, & \xi \leq-1, \\ \frac{1}{2}(\xi+1-\alpha \varepsilon)+\frac{1}{4}(\xi-(1-\alpha) \varepsilon) t, & -1 \leq \xi \leq 1, \\ -\frac{1-\alpha}{2}(\xi-1)+1-\frac{\alpha \varepsilon}{2}+\frac{1}{4}((1-\alpha)(\xi-1-\varepsilon)+1) t, & 1 \leq \xi \leq 1+2 \varepsilon, \\ 1-\varepsilon+\frac{\alpha \varepsilon}{2}+\frac{1}{4}(1+(1-\alpha) \varepsilon) t, & 1+2 \varepsilon \leq \xi\end{cases}
$$

$$
\bar{U}(\xi, t)= \begin{cases}-2 \varepsilon-\frac{1}{4}(1+\varepsilon) t, & \xi \leq-1, \\ \frac{1}{2}(\xi+1)-2 \varepsilon+\frac{1}{4}(\xi-\varepsilon) t, & -1 \leq \xi \leq 1+2 \varepsilon, \\ 1-\varepsilon+\frac{1}{4}(1+\varepsilon) t, & 1+2 \varepsilon \leq \xi\end{cases}
$$

$$
V(\xi, t)= \begin{cases}0, & \xi \leq-1 \\ \frac{1}{2}(\xi+1), & -1 \leq \xi \leq 1 \\ \frac{1-\alpha}{2} \xi+\frac{1+\alpha}{2}, & 1 \leq \xi \leq 1+2 \varepsilon \\ 1+(1-\alpha) \varepsilon, & 1+2 \varepsilon \leq \xi\end{cases}
$$

$\bar{V}(\xi, t)=\bar{H}_{0}(\xi)$,

(A.65)

$H(\xi, t)=H_{0}(\xi)$,

(A.66)

$\bar{H}(\xi, t)=\bar{H}_{0}(\xi)$.

The solutions in Eulerian coordinates for $2 \leq t$ are given by

(A.71) $\rho(x, t)=0$,

$$
\begin{aligned}
& x_{1}(t)=-1+\frac{\alpha \varepsilon}{2}-\frac{\alpha \varepsilon}{2} t-\frac{1}{8}(1+(1-\alpha) \varepsilon) t^{2} \\
& x_{2}(t)=\frac{\alpha \varepsilon}{2}+\left(1-\frac{\alpha \varepsilon}{2}\right) t+\frac{1}{8}(1-(1-\alpha) \varepsilon) t^{2} \\
& x_{3}(t)=\varepsilon-\frac{\alpha \varepsilon}{2}+\left(1-\varepsilon+\frac{\alpha \varepsilon}{2}\right) t+\frac{1}{8}(1+(1-\alpha) \varepsilon) t^{2}
\end{aligned}
$$

$$
u(x, t)= \begin{cases}-\frac{\alpha \varepsilon}{2}-\frac{1}{4}(1+(1-\alpha) \varepsilon) t, & x \leq x_{1}(t), \\ \frac{x-x_{1}(t)}{\left(1+\frac{1}{2} t\right)^{2}}-\frac{\alpha \varepsilon}{2}-\frac{1}{4}(1+(1-\alpha) \varepsilon) t, & x_{1}(t) \leq x \leq x_{2}(t), \\ -\frac{1}{1-\frac{1}{2} t}\left(x-2-\frac{1}{2}(1-\varepsilon) t+\frac{1}{8}(1-\varepsilon) t^{2}\right), & x_{2}(t) \leq x \leq x_{3}(t), \\ 1-\varepsilon+\frac{\alpha \varepsilon}{2}+\frac{1}{4}(1+(1-\alpha) \varepsilon) t, & x_{3}(t) \leq x,\end{cases}
$$

$$
\begin{aligned}
d \nu(t)= & \frac{1}{1+t+\frac{1}{4} t^{2}} \mathbf{1}_{\left[x_{1}(t), x_{2}(t)\right]} d x+\frac{1}{(1-\alpha)\left(1-t+\frac{1}{4} t^{2}\right)} \mathbf{1}_{\left[x_{2}(t), x_{3}(t)\right]} d x \\
& +\varepsilon \delta_{\left(x=2+\frac{1-\varepsilon}{2}, t=2\right)},
\end{aligned}
$$




$$
\begin{aligned}
& d \mu(t)=\frac{1}{1+t+\frac{1}{4} t^{2}} \mathbf{1}_{\left[x_{1}(t), x_{2}(t)\right]} d x+\frac{1}{\left(1-t+\frac{1}{4} t^{2}\right)} \mathbf{1}_{\left[x_{2}(t), x_{3}(t)\right]} d x \\
& +(1-\alpha) \varepsilon \delta_{\left(x=2+\frac{1-\varepsilon}{2}, t=2\right)},
\end{aligned}
$$

(A.76) $\bar{u}(x, t)= \begin{cases}-2 \varepsilon-\frac{1}{4}(1+\varepsilon) t, & x \leq \bar{x}_{1}(t), \\ \frac{1}{1+\frac{1}{2} t}\left(x+1-2 \varepsilon-\frac{1}{4} t(1-3 \varepsilon)\right), & \bar{x}_{1}(t) \leq x \leq \bar{x}_{2}(t), \\ 1-\varepsilon+\frac{1}{4}(1+\varepsilon) t, & \bar{x}_{2}(t) \leq x,\end{cases}$

(A.77) $\bar{\rho}(x, t)=0$,

(A.78) $d \bar{\nu}(t)=\frac{1}{1+t+\frac{1}{4} t^{2}} \mathbf{1}_{\left[\bar{x}_{1}(t), \bar{x}_{2}(t)\right]} d x$,

(A.79) $\quad \bar{\mu}(t)=\bar{\nu}(t)$,

The curves tracking the break points, $x_{1}, x_{2}, x_{3}, \bar{x}_{1}$, and $\bar{x}_{2}$, is drawn in Figure 4. In Figure 5 the cumulative function of the measures $\mu$ and $\bar{\mu}$ are plotted for $t=0,2,4$. Note that they do not coincide after wave breaking.

For $t<2$ the norms of $X(t)-\bar{X}(t)$ are given by

$$
\begin{aligned}
\|y(t)-\bar{y}(t)\|_{\infty} & =2 \varepsilon t, \\
\|U(t)-\bar{U}(t)\|_{\infty} & =2 \varepsilon, \\
\left\|y_{\xi}(t)-\bar{y}_{\xi}(t)\right\|_{2} & =\sqrt{2 \varepsilon}, \\
\left\|U_{\xi}(t)-\bar{U}_{\xi}(t)\right\|_{2} & =\sqrt{2 \varepsilon}, \\
\left\|V_{\xi}(t)-\bar{V}_{\xi}(t)\right\|_{2} & =0, \\
\|g(X(t))-g(\bar{X})\|_{2} & =\sqrt{2 \varepsilon}\left(t+\frac{\alpha}{2}\right), \\
\left\|g_{2}(X(t))-g_{2}(\bar{X}(t))\right\|_{2} & =0, \\
\left\|g_{3}(X(t))-g_{3}(\bar{X}(t))\right\|_{2} & =0, \\
\left\|\alpha^{\prime}\right\|_{\infty}\left\|U(t) H_{0, \xi}-\bar{U}(t) \bar{H}_{0, \xi}\right\|_{2} & =0,
\end{aligned}
$$

while for $2 \leq t$ we have

$$
\begin{aligned}
\|y(t)-\bar{y}(t)\|_{\infty} & =\varepsilon\left(2 t+\frac{\alpha}{8}(t-2)^{2}\right), \\
\|U(t)-\bar{U}(t)\|_{\infty} & =2 \varepsilon+\frac{\alpha \varepsilon}{4}(t-2), \\
\left\|y_{\xi}(t)-\bar{y}_{\xi}(t)\right\|_{2} & =\sqrt{2 \varepsilon}\left(t+\frac{\alpha}{8}(t-2)^{2}\right), \\
\left\|U_{\xi}(t)-\bar{U}_{\xi}(t)\right\|_{2} & =\sqrt{2 \varepsilon}\left(1+\frac{\alpha}{4}(t-2)\right), \\
\left\|V_{\xi}(t)-\bar{V}_{\xi}(t)\right\|_{2} & =\sqrt{2 \varepsilon} \frac{\alpha}{2} \\
\|g(X)-g(\bar{X})\|_{2} & =\sqrt{2 \varepsilon}\left(\frac{\alpha}{2}+t+\frac{\alpha}{8}(t-2)^{2}\right), \\
\left\|g_{2}(X(t))-g_{2}(\bar{X}(t))\right\|_{2} & =0 \\
\left\|g_{3}(X(t))-g_{3}(\bar{X}(t))\right\|_{2} & =0
\end{aligned}
$$




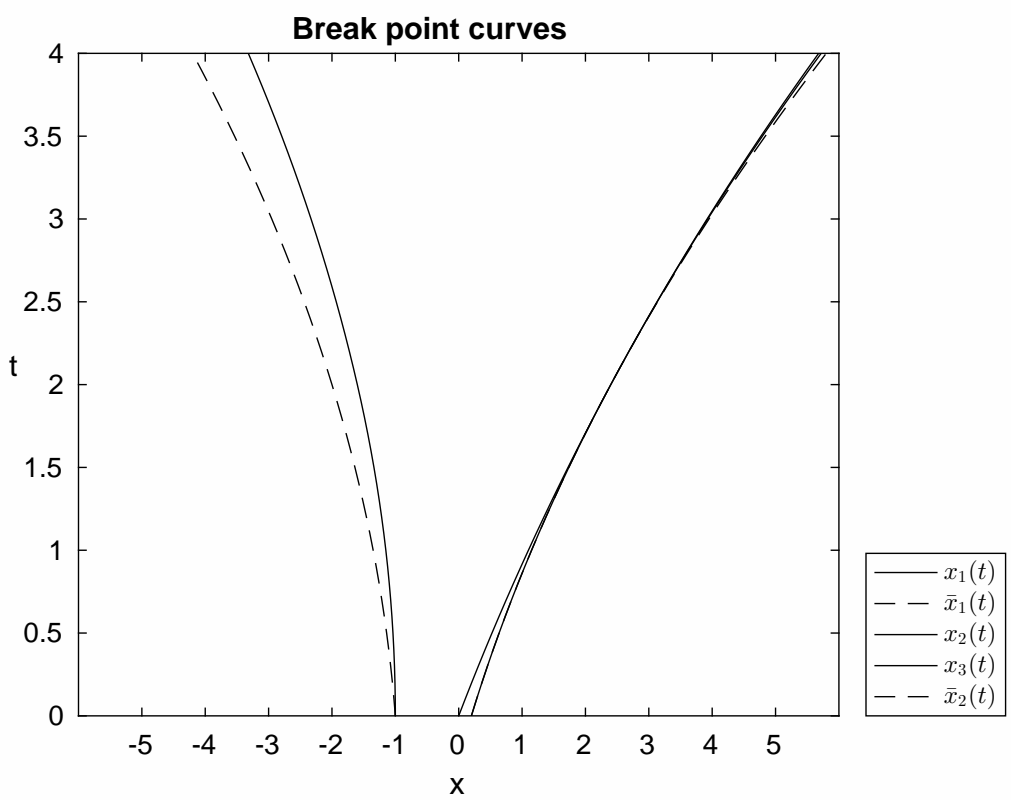

Figure 4. A plot of characteristic curves $x_{i}(\xi, t), i=1,2,3$ and $\bar{x}_{i}(t), t=1,2$ from (A.67) in Example A.2.

$$
\left\|\alpha^{\prime}\right\|_{\infty}\left\|U(t) H_{0, \xi}-\bar{U}(t) \bar{H}_{0, \xi}\right\|_{2}=0 .
$$

That is, at $t=2$ the norm $\left\|U_{\xi}(t)-\bar{U}_{\xi}(t)\right\|_{2}$ suddenly starts to grow, and the growth depends on $\alpha$. Moreover, there is a jump in $\left\|V_{\xi}(t)-\bar{V}_{\xi}(t)\right\|_{2}$ at $t=2$, so that term cannot be a part of the metric. Note that since $H_{0}=\bar{H}_{0}$ all terms involving differences in $\mathrm{H}$ or $H_{\xi}$ vanish.

Example A.3. In this example we demonstrate the iteration scheme from the proof of Lemma [2.3. In particular we illustrate how the scheme reaches the limit in a finite number of iterations for multipeakon initial data. Let $X_{0} \in \mathcal{F}_{0}$ be given by

$$
\begin{gathered}
y_{0}(\xi)= \begin{cases}\xi, & \xi \leq-1, \\
\frac{1}{2}(\xi-1), & -1 \leq \xi \leq 1, \\
\frac{4}{5}(\xi-1), & 1 \leq \xi \leq \frac{7}{2}, \\
\xi-\frac{3}{2}, & \frac{7}{2} \leq \xi\end{cases} \\
U_{0}(\xi)= \begin{cases}1, & \xi \leq-1, \\
-\frac{1}{2}(\xi-1), & -1 \leq \xi \leq 1, \\
-\frac{2}{5}(\xi-1), & 1 \leq \xi \leq \frac{7}{2}, \\
-1, & \frac{7}{2} \leq \xi,\end{cases}
\end{gathered}
$$$$
H_{0}(\xi)= \begin{cases}0, & \xi \leq-1, \\ \frac{1}{2}(\xi+1), & -1 \leq \xi \leq 1, \\ \frac{1}{5}(\xi+4), & 1 \leq \xi \leq \frac{7}{2}, \\ \frac{3}{2}, & \frac{7}{2} \leq \xi\end{cases}
$$

$$
V_{0}=H_{0},
$$



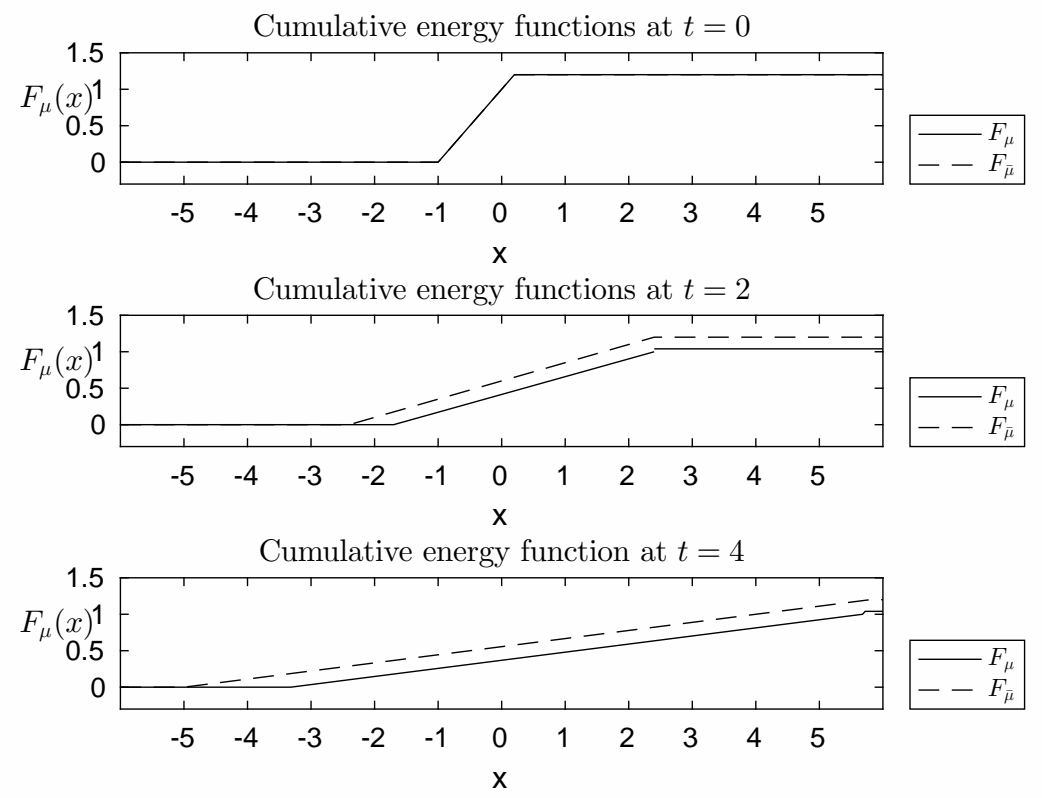

Figure 5. Plots of the cumulative energy functions in Example A.2 for $t=0,2,4$.

$$
r_{0}=0,
$$

which gives

$$
\tau(\xi)= \begin{cases}\infty, & \xi \leq-1 \\ 2, & -1 \leq \xi \leq 1 \\ 4, & 1 \leq \xi \leq \frac{7}{2} \\ \infty, & \frac{7}{2} \leq \xi\end{cases}
$$

Choose $\alpha(x)$ such that

$$
\alpha(x)= \begin{cases}0, & x \leq 0, \\ \frac{1}{4} x, & 0 \leq x \leq 3, \\ \frac{3}{4}, & 3 \leq x .\end{cases}
$$

Then, in the notation of the proof of Lemma 2.3, we have $X_{1}(t)=X_{0}$. For $t<2$ the next term is given by

$$
\begin{aligned}
& y_{2}(\xi, t)= \begin{cases}\xi+t-\frac{3}{16} t^{2}, & \xi \leq-1, \\
\frac{1}{2}(\xi-1)-\frac{1}{2}(\xi-1) t+\frac{1}{8}\left(\xi-\frac{1}{2}\right) t^{2}, & -1 \leq \xi \leq 1, \\
\frac{4}{5}(\xi-1)-\frac{2}{5}(\xi-1) t+\frac{1}{20}\left(\xi+\frac{1}{4}\right) t^{2}, & 1 \leq \xi \leq \frac{7}{2}, \\
\xi-\frac{3}{2}-t+\frac{3}{16} t^{2}, & \frac{7}{2} \leq \xi,\end{cases} \\
& U_{2}(\xi, t)= \begin{cases}1-\frac{3}{8} t, & \xi \leq-1, \\
-\frac{1}{2}(\xi-1)+\frac{1}{4}\left(\xi-\frac{1}{2}\right) t, & -1 \leq \xi \leq 1, \\
-\frac{2}{5}(\xi-1)+\frac{1}{10}\left(\xi+\frac{1}{4}\right) t, & 1 \leq \xi \leq \frac{7}{2}, \\
-1+\frac{3}{8} t, & \frac{7}{2} \leq \xi,\end{cases}
\end{aligned}
$$




$$
H_{2}(\xi, t)= \begin{cases}0, & \xi \leq-1, \\ \frac{1}{2}(\xi+1), & -1 \leq \xi \leq 1, \\ \frac{1}{5}(\xi+4), & 1 \leq \xi \leq \frac{7}{2}, \\ \frac{3}{2}, & \frac{7}{2} \leq \xi,\end{cases}
$$

$$
\begin{aligned}
& V_{2}(t)=H_{0}, \\
& r_{2}(t)=0 .
\end{aligned}
$$

The amount of energy dissipated at $t=2$ is given by

$$
\alpha\left(y_{1}(\xi, \tau(\xi))\right)=0, \quad-1 \leq \xi \leq 1,
$$

and hence we have for $2 \leq t<4$ that

$$
\begin{aligned}
& y_{2}(\xi, t)= \begin{cases}\xi+t-\frac{3}{16} t^{2}, & \xi \leq-1, \\
\frac{1}{2}(\xi-1)-\frac{1}{2}(\xi-1) t+\frac{1}{8}\left(\xi-\frac{1}{2}\right) t^{2}, & -1 \leq \xi \leq 1, \\
\frac{4}{5}(\xi-1)-\frac{2}{5}(\xi-1) t+\frac{1}{20}\left(\xi+\frac{1}{4}\right) t^{2}, & 1 \leq \xi \leq \frac{7}{2}, \\
\xi-\frac{3}{2}-t+\frac{3}{16} t^{2}, & \frac{7}{2} \leq \xi,\end{cases} \\
& U_{2}(\xi, t)= \begin{cases}1-\frac{3}{8} t, & \xi \leq-1, \\
-\frac{1}{2}(\xi-1)+\frac{1}{4}\left(\xi-\frac{1}{2}\right) t, & -1 \leq \xi \leq 1, \\
-\frac{2}{5}(\xi-1)+\frac{1}{10}\left(\xi+\frac{1}{4}\right) t, & 1 \leq \xi \leq \frac{7}{2}, \\
-1+\frac{3}{8} t, & \frac{7}{2} \leq \xi,\end{cases} \\
& H_{2}(\xi, t)= \begin{cases}0, & \xi \leq-1, \\
\frac{1}{2}(\xi+1), & -1 \leq \xi \leq 1, \\
\frac{1}{5}(\xi+4), & 1 \leq \xi \leq \frac{7}{2}, \\
\frac{3}{2}, & \frac{7}{2} \leq \xi,\end{cases} \\
& V_{2}(t)=H_{0}, 0 .
\end{aligned}
$$

The amount dissipated at $t=4$ is given by

$$
\alpha\left(y_{1}(\xi, \tau(\xi))\right)=\frac{1}{5}(\xi-1), \quad 1 \leq \xi \leq \frac{7}{2} .
$$

Thus, for $t \geq 4$,

$$
\begin{gathered}
y_{2}(\xi, t)= \begin{cases}\xi+1-\frac{1}{2}(t-4)-\frac{11}{64}(t-4)^{2}, & \xi \leq-1, \\
\frac{1}{2}(\xi+1)+\frac{1}{2} \xi(t-4)+\frac{1}{8}\left(\xi-\frac{3}{8}\right)(t-4)^{2}, & -1 \leq \xi \leq 1, \\
1+\frac{1}{2}(t-4)+\frac{1}{200}\left(\frac{37}{8}+12 \xi-\xi^{2}\right)(t-4)^{2}, & 1 \leq \xi \leq \frac{7}{2}, \\
\xi-\frac{5}{2}+\frac{1}{2}(t-4)+\frac{11}{64}(t-4)^{2}, & \frac{7}{2} \leq \xi,\end{cases} \\
U_{2}(\xi, t)= \begin{cases}-\frac{1}{2}-\frac{11}{32}(t-4), & \xi \leq-1, \\
\frac{1}{2} \xi+\frac{1}{4}\left(\xi-\frac{3}{8}\right)(t-4), & -1 \leq \xi \leq 1, \\
\frac{1}{2}+\frac{1}{100}\left(\frac{37}{8}+12 \xi-\xi^{2}\right)(t-4), & 1 \leq \xi \leq \frac{7}{2}, \\
\frac{1}{2}+\frac{11}{32}(t-4), & \frac{7}{2} \leq \xi,\end{cases}
\end{gathered}
$$


(A.119)

$$
H_{2}(\xi, t)= \begin{cases}0, & \xi \leq-1 \\ \frac{1}{2}(\xi+1), & -1 \leq \xi \leq 1 \\ \frac{1}{5}(\xi+4), & 1 \leq \xi \leq \frac{7}{2} \\ \frac{3}{2}, & \frac{7}{2} \leq \xi\end{cases}
$$

(A.120) $V_{2}(\xi, t)= \begin{cases}0, & \xi \leq-1, \\ \frac{1}{2}(\xi+1), & -1 \leq \xi \leq 1, \\ \frac{1}{50}\left(39+12 \xi-\xi^{2}\right), & 1 \leq \xi \leq \frac{7}{2}, \\ \frac{11}{8}, & \frac{7}{2} \leq \xi,\end{cases}$

(A.121) $\quad r_{2}(t)=0$.

Then

$$
\alpha\left(y_{2}(\xi, \tau(\xi))\right)= \begin{cases}\frac{1}{16}, & -1 \leq \xi \leq 1 \\ \frac{1}{4}, & 1 \leq \xi \leq \frac{7}{2}\end{cases}
$$

and $X_{3}(t)$ can be computed as follows. For $t<2$ we have $X_{3}(t)=X_{2}(t)$. For $2 \leq t<4$ we have,

(A.123)

$$
y_{3}(\xi, t)= \begin{cases}\xi+\frac{5}{4}+\frac{1}{4}(t-2)-\frac{23}{128}(t-2)^{2}, & \xi \leq-1, \\ \frac{1}{4}+\frac{1}{4}(t-2)+\frac{1}{128}(15 \xi-8)(t-2)^{2}, & -1 \leq \xi \leq 1, \\ \frac{1}{5}\left(\xi+\frac{1}{4}\right)-\frac{1}{5}\left(\xi-\frac{9}{4}\right)(t-2)+\frac{1}{20}\left(\xi+\frac{3}{32}\right)(t-2)^{2}, & 1 \leq \xi \leq \frac{7}{2}, \\ \xi-\frac{11}{4}-\frac{1}{4}(t-2)+\frac{23}{128}(t-2)^{2}, & \frac{7}{2} \leq \xi,\end{cases}
$$

$$
U_{3}(\xi, t)= \begin{cases}\frac{1}{4}-\frac{23}{64}(t-2), & \xi \leq-1 \\ \frac{1}{4}+\frac{1}{8}\left(\frac{15}{8} \xi-1\right)(t-2), & -1 \leq \xi \leq 1 \\ -\frac{1}{5}\left(\xi-\frac{9}{4}\right)+\frac{1}{10}\left(\xi+\frac{3}{32}\right)(t-2), & 1 \leq \xi \leq \frac{7}{2} \\ -\frac{1}{4}+\frac{23}{64}(t-2), & \frac{7}{2} \leq \xi\end{cases}
$$

$$
H_{3}(\xi, t)= \begin{cases}0, & \xi \leq-1 \\ \frac{1}{2}(\xi+1), & -1 \leq \xi \leq 1 \\ \frac{1}{5}(\xi+4), & 1 \leq \xi \leq \frac{7}{2} \\ \frac{3}{2}, & \frac{7}{2} \leq \xi\end{cases}
$$

$$
V_{3}(\xi, t)= \begin{cases}0, & \xi \leq-1, \\ \frac{15}{32}(\xi+1), & -1 \leq \xi \leq 1, \\ \frac{1}{5} \xi+\frac{59}{80}, & 1 \leq \xi \leq \frac{7}{2} \\ \frac{23}{16}, & \frac{7}{2} \leq \xi\end{cases}
$$

(A.127) $r_{3}(t)=0$. 
At $t=4$ there is wave breaking for $1<\xi<\frac{7}{2}$, and one fourth of the energy is dissipated. Thus for $t \geq 4$ we have

$$
\begin{aligned}
& y_{3}(\xi, t)= \begin{cases}\xi+\frac{33}{32}-\frac{15}{32}(t-4)-\frac{21}{128}(t-4)^{2}, & \xi \leq-1, \\
\frac{15}{32} \xi+\frac{1}{2}+\frac{15}{32} \xi(t-4)+\frac{1}{128}(15 \xi-6)(t-4)^{2}, & -1 \leq \xi \leq 1, \\
\frac{31}{32}+\frac{15}{32}(t-4)+\frac{3}{80}\left(\xi+\frac{7}{8}\right)(t-4)^{2}, & 1 \leq \xi \leq \frac{7}{2}, \\
\xi-\frac{81}{32}+\frac{15}{32}(t-4)+\frac{21}{128}(t-4)^{2}, & \frac{7}{2} \leq \xi,\end{cases} \\
& U_{3}(\xi, t)= \begin{cases}-\frac{15}{32}-\frac{21}{64}(t-4), & \xi \leq-1, \\
\frac{15}{32} \xi+\frac{1}{64}(15 \xi-6)(t-4), & -1 \leq \xi \leq 1, \\
\frac{15}{32}+\frac{3}{40}\left(\xi+\frac{7}{8}\right)(t-4), & 1 \leq \xi \leq \frac{7}{2} \\
\frac{15}{32}+\frac{21}{64}(t-4), & \frac{7}{2} \leq \xi\end{cases} \\
& H_{3}(\xi, t)= \begin{cases}0, & \xi \leq-1 \\
\frac{1}{2}(\xi+1), & -1 \leq \xi \leq 1 \\
\frac{1}{5}(\xi+4), & 1 \leq \xi \leq \frac{7}{2} \\
\frac{3}{2}, & \frac{7}{2} \leq \xi\end{cases} \\
& V_{3}(\xi, t)= \begin{cases}0, & \xi \leq-1 \\
\frac{15}{32}(\xi+1), & -1 \leq \xi \leq 1 \\
\frac{3}{20}\left(\xi+\frac{21}{4}\right), & 1 \leq \xi \leq \frac{7}{2} \\
\frac{21}{16}, & \frac{7}{2} \leq \xi\end{cases} \\
& \text { (A.132) } \quad r_{3}(t)=0 \text {. }
\end{aligned}
$$

Then

$$
\alpha\left(y_{3}(\xi, \tau(\xi))\right)= \begin{cases}\frac{1}{16}, & -1 \leq \xi \leq 1, \\ \frac{31}{128}, & 1 \leq \xi \leq \frac{7}{2},\end{cases}
$$

and we have that $X_{4}(t)=X_{3}(t)$ for $t<4$. When $t \geq 4$, then

$$
y_{4}(\xi, t)= \begin{cases}\xi+\frac{33}{32}-\frac{15}{32}(t-4)-\frac{337}{2048}(t-4)^{2}, & \xi \leq-1, \\ \frac{15}{32} \xi+\frac{1}{2}+\frac{15}{32} \xi(t-4)+\frac{1}{2048}(240 \xi-97)(t-4)^{2}, & -1 \leq \xi \leq 1, \\ \frac{31}{32}+\frac{15}{32}(t-4)+\frac{1}{10240}(388 \xi+327)(t-4)^{2}, & 1 \leq \xi \leq \frac{7}{2}, \\ \xi-\frac{81}{32}+\frac{15}{32}(t-4)+\frac{337}{2048}(t-4)^{2}, & \frac{7}{2} \leq \xi,\end{cases}
$$

$$
U_{4}(\xi, t)= \begin{cases}-\frac{15}{32}-\frac{337}{102}(t-4), & \xi \leq-1 \\ \frac{15}{32} \xi+\frac{1}{1024}(240 \xi-97)(t-4), & -1 \leq \xi \leq 1 \\ \frac{15}{32}+\frac{1}{5120}(388 \xi+327)(t-4), & 1 \leq \xi \leq \frac{7}{2} \\ \frac{15}{32}+\frac{337}{1024}(t-4), & \frac{7}{2} \leq \xi\end{cases}
$$

$$
H_{4}(\xi, t)= \begin{cases}0, & \xi \leq-1 \\ \frac{1}{2}(\xi+1), & -1 \leq \xi \leq 1 \\ \frac{1}{5}(\xi+4), & 1 \leq \xi \leq \frac{7}{2} \\ \frac{3}{2}, & \frac{7}{2} \leq \xi\end{cases}
$$




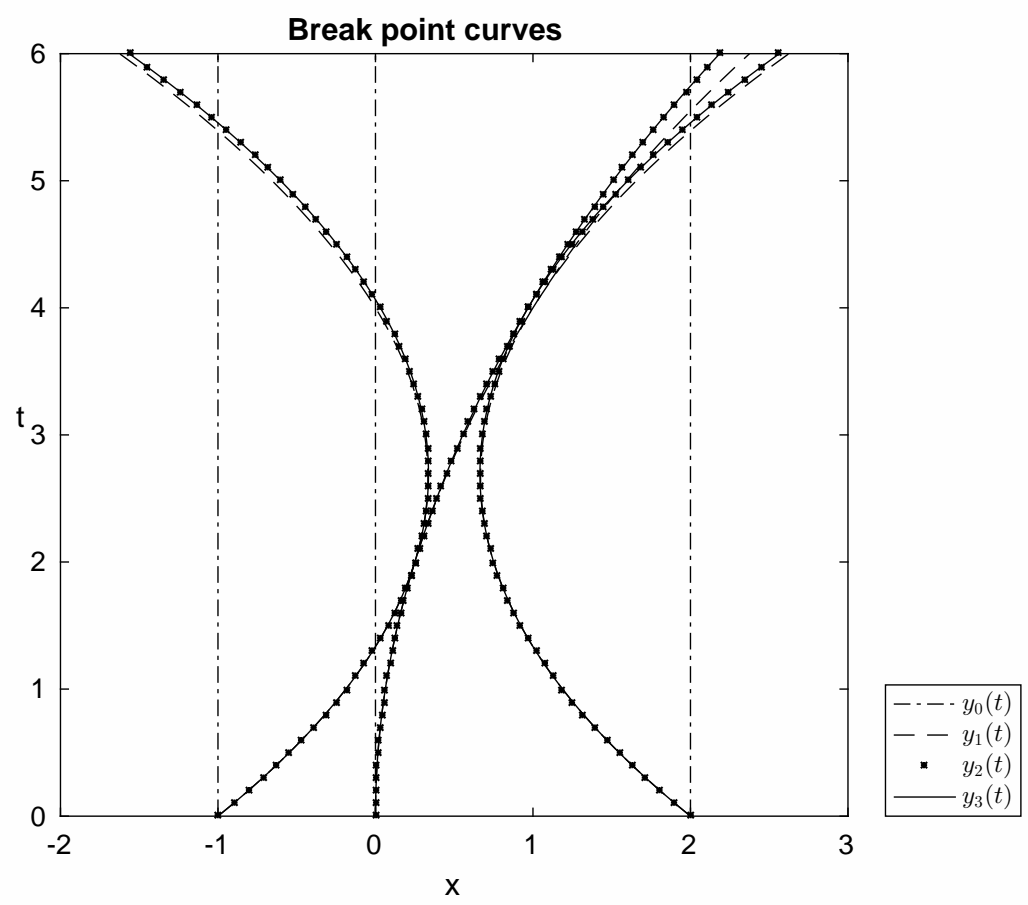

FiguRE 6. A plot of characteristic curves $y_{i}(\xi, t), i=1,2,3,4$ in Example A.3 corresponding to $\xi=-1, \xi=1$, and $\xi=\frac{7}{2}$ from left to right.

(A.137)

$$
V_{4}(\xi, t)= \begin{cases}0, & \xi \leq-1 \\ \frac{15}{32}(\xi+1), & -1 \leq \xi \leq 1, \\ \frac{1}{640}(97 \xi+503), & 1 \leq \xi \leq \frac{7}{2} \\ \frac{337}{256}, & \frac{7}{2} \leq \xi\end{cases}
$$

(A.138) $r_{4}(t)=0$.

Since $X_{5}(t)=X_{4}(t)$ for all $t>0$, the $\alpha$-dissipative solution with initial data $X_{0}$ at time $t$ is given by $X_{5}(t)$. In Figure [6 $y_{i}(\xi, t)$ has been plotted for $i=1,2,3,4$ and $\xi=-1,1, \frac{7}{2}$.

Example A.4. In this example we demonstrate why it is necessary to restrict alpha to $[0,1)$. Select $\alpha$ such that $\alpha\left(\frac{1}{4}\right)=1$, and $\alpha\left(\frac{1}{2}\right)=\frac{1}{2}$. Let $\left(u_{0}, \rho_{0}, \nu_{0}, \mu_{0}\right)$ be given by $\rho_{0}=0, \nu_{0}=\mu_{0}=\left(\nu_{0}\right)_{a c}$, and

$$
u_{0}(x)= \begin{cases}1, & x \leq-1 \\ -x, & -1 \leq x \leq 0 \\ -\frac{1}{2} x, & 0 \leq x \leq 2 \\ -1, & 2 \leq x\end{cases}
$$


Then $X_{0}=L\left(\left(u_{0}, \rho_{0}, \nu_{0}, \mu_{0}\right)\right)$ is given by

$$
\begin{aligned}
y_{0}(\xi) & = \begin{cases}\xi, & \xi \leq-1, \\
\frac{1}{2}(\xi-1), & -1 \leq \xi \leq 1, \\
\frac{4}{5}(\xi-1), & 1 \leq \xi \leq \frac{7}{2}, \\
\xi-\frac{3}{2}, & \frac{7}{2} \leq \xi,\end{cases} \\
U_{0}(\xi) & = \begin{cases}1, & \xi \leq-1, \\
-\frac{1}{2}(\xi-1), & -1 \leq \xi \leq 1, \\
-\frac{2}{5}(\xi-1), & 1 \leq \xi \leq \frac{7}{2}, \\
-1, & \frac{7}{2} \leq \xi,\end{cases} \\
H_{0}(\xi) & = \begin{cases}0, & \xi \leq-1, \\
\frac{1}{2}(\xi+1), & -1 \leq \xi \leq 1, \\
\frac{1}{5}(\xi+4), & 1 \leq \xi \leq \frac{7}{2}, \\
\frac{3}{2}, & \frac{7}{2} \leq \xi,\end{cases} \\
V_{0} & =H_{0}, \\
r_{0} & =0 .
\end{aligned}
$$

We claim that $L \circ M \circ S_{4}\left(X_{0}\right) \neq \Pi \circ S_{4}\left(X_{0}\right)$. Note that $S_{4}\left(X_{0}\right)$ does not belong to the set $\mathcal{F}^{\alpha}$, since we choose an invalid $\alpha$. However the mappings $L$ and $M$ can still be applied in this more general case. For $t=4, S_{4}\left(X_{0}\right)$ reads

$$
\begin{aligned}
y(\xi, 4) & = \begin{cases}\xi+\frac{3}{2}, & \xi \leq-1, \\
\frac{1}{2}, & -1 \leq \xi \leq \frac{7}{2}, \\
\xi-3, & \frac{7}{2} \leq \xi\end{cases} \\
U(\xi, 4) & =0
\end{aligned}
$$$$
H(\xi, 4)= \begin{cases}0, & \xi \leq-1, \\ \frac{1}{2}(\xi+1), & -1 \leq \xi \leq 1, \\ \frac{1}{5}(\xi+4), & 1 \leq \xi \leq \frac{7}{2}, \\ \frac{3}{2}, & \frac{7}{2} \leq \xi\end{cases}
$$

$$
V(\xi, 4)= \begin{cases}0, & \xi \leq 1, \\ \frac{1}{10}(\xi-1), & 1 \leq \xi \leq \frac{7}{2}, \\ \frac{1}{4}, & \frac{7}{2} \leq \xi .\end{cases}
$$

ad $\tilde{X}=\Pi \circ S_{4}\left(X_{0}\right)$ is given by

$$
\begin{gathered}
\tilde{y}(\xi)= \begin{cases}\xi, & \xi \leq \frac{1}{2}, \\
\frac{1}{2}, & \frac{1}{2} \leq \xi \leq 2, \\
\xi-\frac{3}{2}, & 2 \leq \xi\end{cases} \\
\tilde{U}(\xi)=0, \\
\tilde{H}(\xi)= \begin{cases}0, & \xi \leq \frac{1}{2}, \\
\xi-\frac{1}{2}, & \frac{1}{2} \leq \xi \leq 2, \\
\frac{3}{2}, & 2 \leq \xi,\end{cases}
\end{gathered}
$$




$$
\tilde{V}(\xi)= \begin{cases}0, & \xi \leq \frac{3}{2}, \\ \frac{1}{2} \xi-\frac{3}{4}, & \frac{3}{2} \leq \xi \leq 2, \\ \frac{1}{4}, & 2 \leq \xi\end{cases}
$$

On the other hand let $\bar{X}=L \circ M \circ S_{4}\left(X_{0}\right)$, then we get

$$
\begin{gathered}
\bar{y}(\xi)= \begin{cases}\xi, & \xi \leq \frac{1}{2}, \\
\frac{1}{2}, & \frac{1}{2} \leq \xi \leq 2, \\
\xi-\frac{3}{2}, & 2 \leq \xi,\end{cases} \\
\bar{U}(\xi)=0, \\
\bar{H}(\xi)= \begin{cases}0, & \xi \leq \frac{1}{2}, \\
\xi-\frac{1}{2}, & \frac{1}{2} \leq \xi \leq 2, \\
\frac{3}{2}, & 2 \leq \xi,\end{cases} \\
\bar{V}(\xi)= \begin{cases}0, & \xi \leq \frac{1}{2}, \\
\frac{1}{6} \xi-\frac{1}{12}, & \frac{1}{2} \leq \xi \leq 2, \\
\frac{1}{4}, & 2 \leq \xi .\end{cases}
\end{gathered}
$$

Since $\tilde{V} \neq \bar{V}$ we have that if we allow that $\alpha(x)=1$ for some $x$ and $\alpha(\bar{x})<1$ for some other $\bar{x}$, then we cannot guarantee that $L \circ M=\mathrm{id}_{\mathcal{F}_{0}}$. By passing to Eulerian coordinates and back some information about $V$ is lost. In Figure 8 the cumulative function for $\nu$ is plotted at $t=0,2,4$. We see that the jump at $t=4$ contains two jumps, one created at $t=2$, the other at $t=4$. Uniqueness is lost for $t>4$ since we are unable to separate the jumps in a systematic way. This can be seen from Figure 9 where cumulative functions for $\nu$ and $\bar{\nu}$ have been plotted at $t=6$. The measure $\nu$ is obtained by $M \circ S_{6} \circ L$, while $\bar{\nu}$ is obtained by $M \circ S_{2} \circ L \circ M \circ S_{4} \circ L$. It should be pointed out that non-uniquenss after wave breaking for $\nu$ does not affect $(u, \rho, \mu)$ in any way.

\section{REFERENCES}

[1] A. Bressan and A. Constantin. Global solutions of the Hunter-Saxton equation. SIAM J. Math. Anal., 37(3):996-1026, 2005.

[2] A. Bressan and A. Constantin. Global conservative solutions of the Camassa-Holm equation. Arch. Ration. Mech. Anal., 183(2):215-239, 2007.

[3] A. Bressan and A. Constantin. Global dissipative solutions of the Camassa-Holm equation. Anal. Appl., 05(01):1-27, 2007.

[4] A. Bressan, H. Holden, and X. Raynaud. Lipschitz metric for the Hunter-Saxton equation. J. Math. Pures Appl., 94(1):68-92, 2010.

[5] A. Bressan, P. Zhang, and Y. Zheng. Asymptotic variational wave equations. Arch. Ration. Mech. Anal., 183(1):163-185, 2007.

[6] R. Camassa and D. D. Holm. An integrable shallow water equation with peaked solitons. Phys. Rev. Lett., 71:1661-1664, Sep 1993.

[7] C. M. Dafermos. Generalized characteristics and the Hunter-Saxton equation. J. Hyperbolic Differ. Equ., 8(01):159-168, 2011.

[8] H.-H. Dai and M. V. Pavlov. Transformations for the Camassa-Holm equation, its highfrequency limit and the Sinh-Gordon equation. J. Phys. Soc. Japan, 67(11):3655-3657, 1998.

[9] K. Grunert, H. Holden, and X. Raynaud. Global solutions for the two-component CamassaHolm system. Comm. Partial Differential Equations, 37(12):2245-2271, 2012.

[10] K. Grunert, H. Holden, and X. Raynaud. Lipschitz metric for the Camassa-Holm equation on the line. Discrete Contin. Dyn. Syst., 33(7):2809-2827, 2013. 


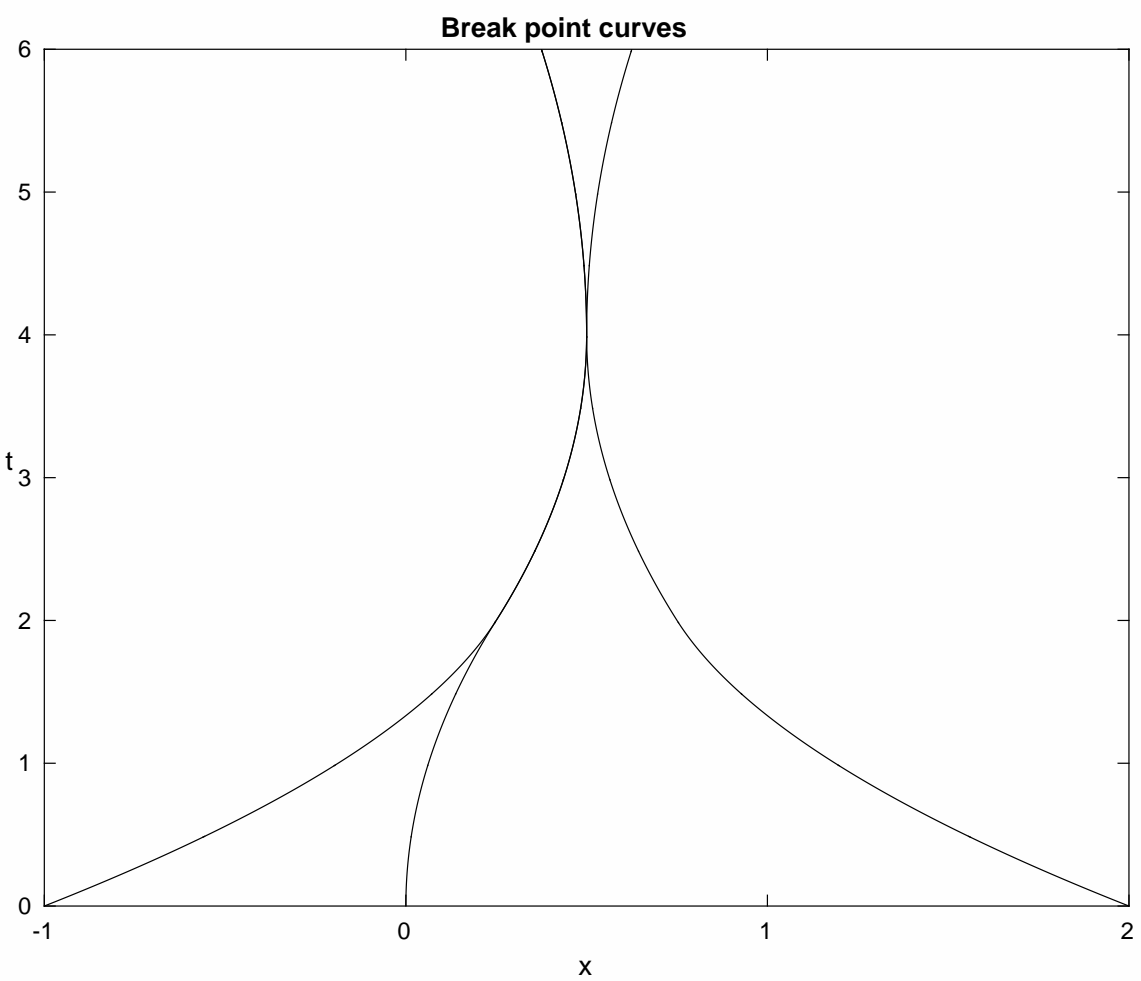

FiguRe 7. A plot of characteristic curves $y(\xi, t)$ corresponding to $\xi=-1, \xi=1$, and $\xi=\frac{7}{2}$ in A.140 in Example A.4 from left to right.

[11] K. Grunert, H. Holden, and X. Raynaud. Lipschitz metric for the two-component CamassaHolm system. In F. Ancona et al., editors, Hyperbolic problems: theory, numerics, applications, volume 8 of AIMS on Applied Mathematics, pages 193-207. AIMS, 2014.

[12] K. Grunert, H. Holden, and X. Raynaud. A continuous interpolation between conservative and dissipative solutions for the two-component Camassa-Holm system. Forum Math. Sigma, 3:e1 (73 pages), 2015.

[13] H. Holden and X. Raynaud. Global conservative solutions of the Camassa-Holm equation-A Lagrangian point of view. Comm. Partial Differential Equations, 32(10):1511-1549, 2007.

[14] J. K. Hunter and R. Saxton. Dynamics of director fields. SIAM J. Appl. Math., 51(6):1498$1521,1991$.

[15] J. K. Hunter and Y. Zheng. On a nonlinear hyperbolic variational equation: I. Global existence of weak solutions. Arch. Ration. Mech. Anal., 129:305-353, 1995.

[16] A. Nordli. A Lipschitz metric for conservative solutions of the two-component Hunter-Saxton system. Methods Appl. Anal. (to appear), 2016.

[17] M. V. Pavlov. The Gurevich-Zybin system. J. Phys. A, 38(17):3823-3840, 2005.

[18] M. Wunsch. The generalized Hunter-Saxton system. SIAM J. Math. Anal., 42(3):1286-1304, 2010.

[19] P. Zhang and Y. Zheng. Existence and uniqueness of solutions of an asymptotic equation arising from a variational wave equation with general data. Arch. Ration. Mech. Anal., 155:49-83, 2000 . 


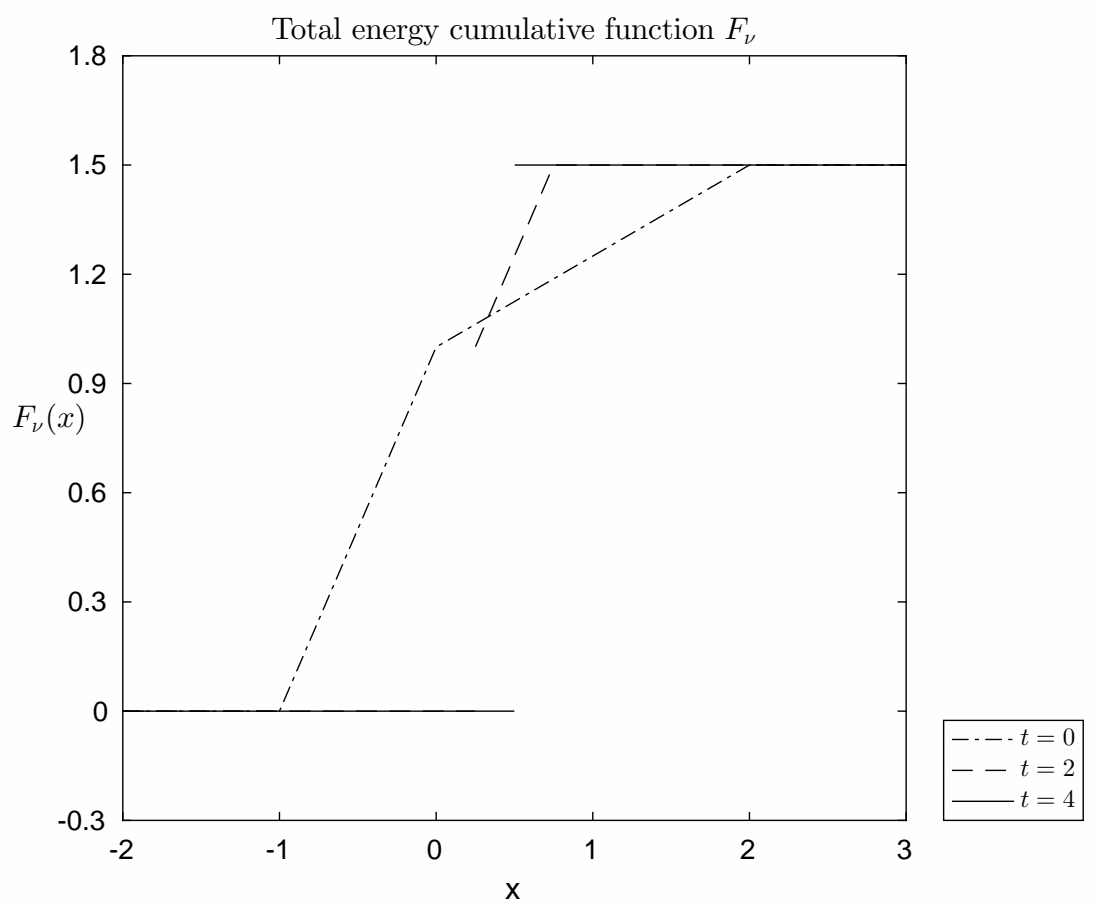

Figure 8. A plot of the cumulative total energy function $F_{\nu}$ in Example A.4 at $t=0, t=2$, and $t=4$.

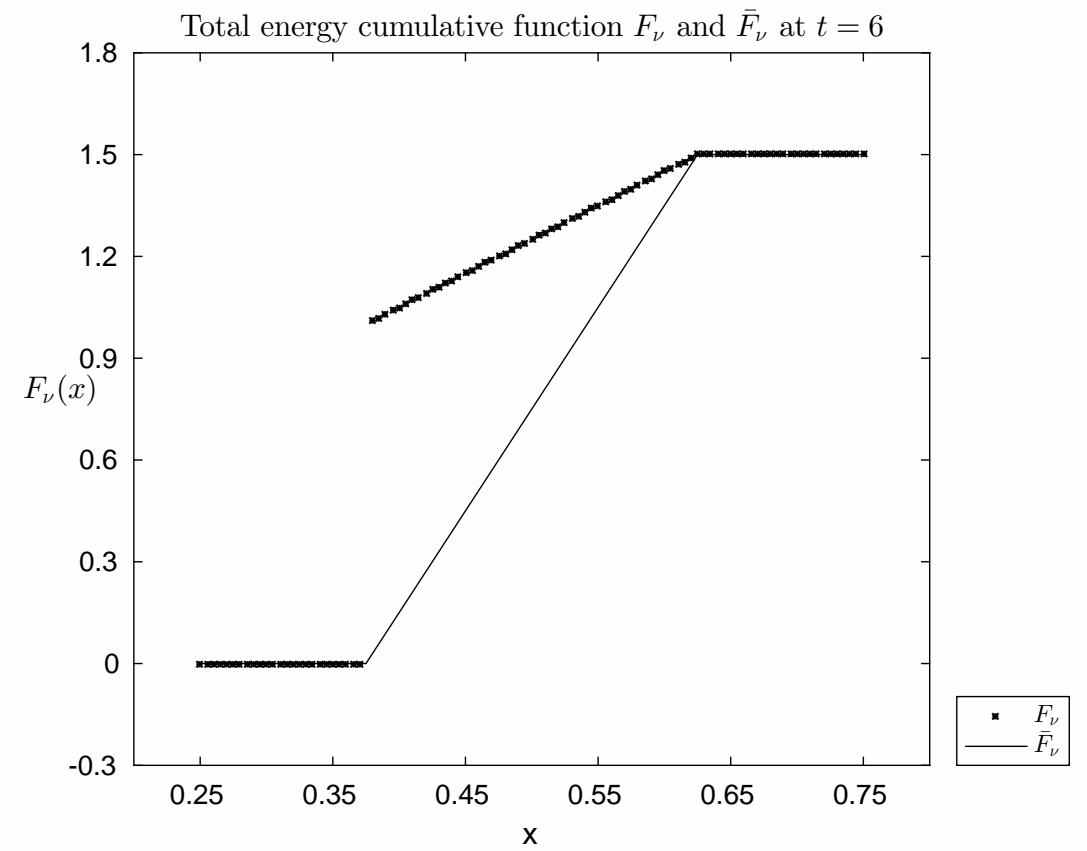

Figure 9. A plot of the cumulative total energy functions $F_{\nu}$ and $F_{\bar{\nu}}$ from Example A.4 at $t=6$. 
Department of Mathematical Sciences, NTnU, Norwegian University of Science and Technology, 7491 Trondheim, Norway

E-mail address: katring@math.ntnu.no

$U R L:$ http://www.math.ntnu.no/ katring/

Department of Mathematical Sciences, NTNU, Norwegian University of Science and Technology, NO-7491 Trondheim, Norway

E-mail address: andenors@math.ntnu.no 